\title{
Quality assurance of 3-D treatment planning systems for external photon and electron beams
}

Practical guidelines for initial verification and periodic quality control of radiation therapy treatment planning systems

\section{NEDERLANDSE COMMISSIE VOOR STRALINGSDOSIMETRIE}

\author{
Report \#\# of the Netherlands Commission on Radiation Dosimetry
}

Authors:

I.A.D. Bruinvis

R.B. Keus

W.J.M. Lenglet

G.J. Meijer

B.J. Mijnheer
A.A. van 't Veld
J.L.M. Venselaar
J. Welleweerd
E. Woudstra

Netherlands Commission on Radiation Dosimetry

Subcommittee Treatment Planning Systems

March 2005 
https://doi.org/10.25030/ncs-015 The NCS report has been downloaded on 26 Apr 2023 


\section{Preface}

The Nederlandse Commissie voor Stralingsdosimetrie (NCS, Netherlands Commission on Radiation Dosimetry) was officially established on September 31982 with the aim of promoting the appropriate use of dosimetry of ionising radiation both for research and practical applications. The NCS is chaired by a board of scientists, installed upon the suggestion of the supporting societies, including the Nederlandse Vereniging voor Radiotherapie en Oncologie (Netherlands Society for Radiotherapy and Oncology), the Nederlandse Vereniging voor Klinische Fysica (Netherlands Society for Medical Physics), the Nederlandse Vereniging voor Radiobiologie (Netherlands Society for Radiobiology), the Nederlandse Vereniging voor Stralingshygiene (Netherlands Society for Radiological Protection), the Nederlandse Vereniging Medische Beeldvorming en Radiotherapie (Netherlands Society of Medical Imaging and Radiotherapy), and the Ministry of Health, Welfare and Sports. To pursue its aims, the NCS accomplishes the following tasks: participation in dosimetry standardisation and promotion of dosimetry intercomparisons, drafting of dosimetry protocols, collection and evaluation of physical data related to dosimetry. Furthermore, the commission shall maintain or establish links with national and international organisations concerned with ionising radiation and promulgate information on new developments in the field of radiation dosimetry.

Current members of the board of the NCS:

Prof.dr. S. Vynckier, chairman

Dr. B.J.M. Heijmen, vice chairman

Ir. W. de Vries, secretary

Dr. J. Zoetelief, treasurer Dr. A.J.J. Bos

Prof.dr. A.A. Lammertsma

Dr. J.M. Schut Dr.ir. F.W. Wittkämper

D. Zweers 


\section{Quality assurance of 3-D treatment planning systems for external photon and electron beams}

Practical guidelines for initial verification and periodic quality control of radiation therapy treatment planning systems

Prepared by the Subcommittee Treatment Planning Systems of the Netherlands Commission on Radiation Dosimetry (NCS). The authors wish to thank Dr. B.A. Fraass of the University of Michigan (USA), chairman of the AAPM Task Group 53 on Quality Assurance for Clinical Radiotherapy Treatment Planning, for his valuable comments.

Members of the Subcommittee:

I.A.D. Bruinvis, chairman ${ }^{1}$

R.B. Keus ${ }^{2}$

W.J.M. Lenglet ${ }^{3}$

G.J. Meijer ${ }^{4}$

B.J. Mijnheer ${ }^{1}$

A.A. van 't Veld ${ }^{5}$

J.L.M. Venselaar ${ }^{6}$

J. Welleweerd ${ }^{7}$

E. Woudstra ${ }^{8}$

\footnotetext{
${ }^{1}$ Het Nederlands Kanker Instituut - Antoni van Leeuwenhoek Ziekenhuis, Amsterdam

${ }^{2}$ Arnhems Radiotherapeutisch Instituut, Arnhem

${ }^{3}$ Radiotherapeutisch Instituut Friesland, Leeuwarden

${ }^{4}$ Catharina Ziekenhuis, Eindhoven

${ }^{5}$ Universitair Medisch Centrum Groningen, Groningen

${ }^{6}$ Dr. Bernard Verbeeten Instituut, Tilburg

${ }^{7}$ Universitair Medisch Centrum Utrecht, Utrecht

${ }^{8}$ Erasmus Medisch Centrum - Daniël den Hoed Oncologisch Centrum, Rotterdam
} 


\section{Contents}

Preface

Pagina nummers toevoegen als document definitief

Title

Contents

Summary

Abbreviations

1. Introduction

1.1 Review of activities

1.2 Aspects of QA of TPS

1.3 Tolerances and accuracy

1.4 Definitions

1.5 Contents of this report

2. Anatomical description

2.1 Basic patient entry

2.2 Image conversion, input and use

2.3 Anatomical structures

3. Beam description

3.1 Beam definition

3.2 Beam display

3.3 Beam geometry

4. Dose calculation

4.1 Beam data input

4.2 Dose calculations

4.3 Monitor unit calculation

5. Plan evaluation and optimisation

5.1 Dose display

5.2 Dose volume histograms

5.3 NTCP and TCP calculations

5.4 Composite dose distributions 
7. Treatment plan description

6.1 Print and plot output

6.2 Electronic data export

6.3 Treatment plan archiving

7. Periodic quality control

7.1 Treatment planning workstation

7.2 Data input and output devices

7.3 Display systems

8. System management and security

8.1 Computer systems

8.2 Computer software

8.3 Computer network

8.4 System security

9. Appendices

A.2.1 CT number representation

A.2.2 Example of phantom

A.2.3 Test of 3-D expansion algorithm

A.4.1 Tolerances for the accuracy of dose calculations

A.4.2 Test configurations

A.5 Computation of sphere volume 
iv

\section{Summary}

Modern 3-D systems for radiation therapy treatment planning have much greater functionality than the old 2-D systems. Therefore the quality assurance (QA) procedures required for initial verification and periodic quality control are much more extensive and complex than with the 2-D systems. In this report practical guidelines for the QA of a 3-D treatment planning system (TPS) are formulated, in order to help the clinics in the design of their QA procedures. The subject of the report is restricted to conventional treatment planning for external photon and electron beam therapy. The report of Task Group 53 of the American Association of Physicists in Medicine (AAPM) was taken as the outline of the QA issues to be addressed; the present report is complementary to the TG53 report. It consists of practical test that cover the essential QA required, each subsection describes a set of suggested tests, preceded by a statement of the scope of the tests and a short explanatory background. The details of a clinic's TPS QA program will depend on the specific TPS and its clinical utilization. This report will be of great practical help, however, in describing general tests that can be applied to a large variety of systems. 
https://doi.org/10.25030/ncs-015 The NCS report has been downloaded on 26 Apr 2023 


\section{Abbreviations}

\begin{tabular}{ll} 
APM & American Association of Physicists in Medicine \\
AP & anterior superior \\
BEV & beam's eye view \\
BIR & British Institute of Radiology \\
C-axis & central axis \\
CCW & counter clockwise \\
cGy & centiGray \\
CT & computed tomography \\
CW & clockwise \\
DICOM & digital imaging and communication in medicine \\
DRR & digitally reconstructed radiograph \\
DVH & dose volume histogram \\
EOS & Elekta Oncology Systems \\
ESTRO & European Society for Therapeutic Radiology and Oncology \\
FOV & field of view \\
Gy & Gray \\
HU & Hounsfield units \\
IAEA & International Atomic Energy Agency \\
ICRU & International Commission on Radiological Units and Measurements \\
ID & Identification \\
IEC & International Electro technical Commission \\
IPEM & Institute of Physics and Engineering in Medicine \\
IPEMB & Institute of Physics and Engineering in Medicine and Biology \\
IMRT & intensity modulated radiation therapy \\
L, W & level, window \\
MLC & multi leaf collimator \\
MR & magnetic resonance \\
MRI & magnetic resonance imaging \\
MU & Monitor unit \\
\hline
\end{tabular}




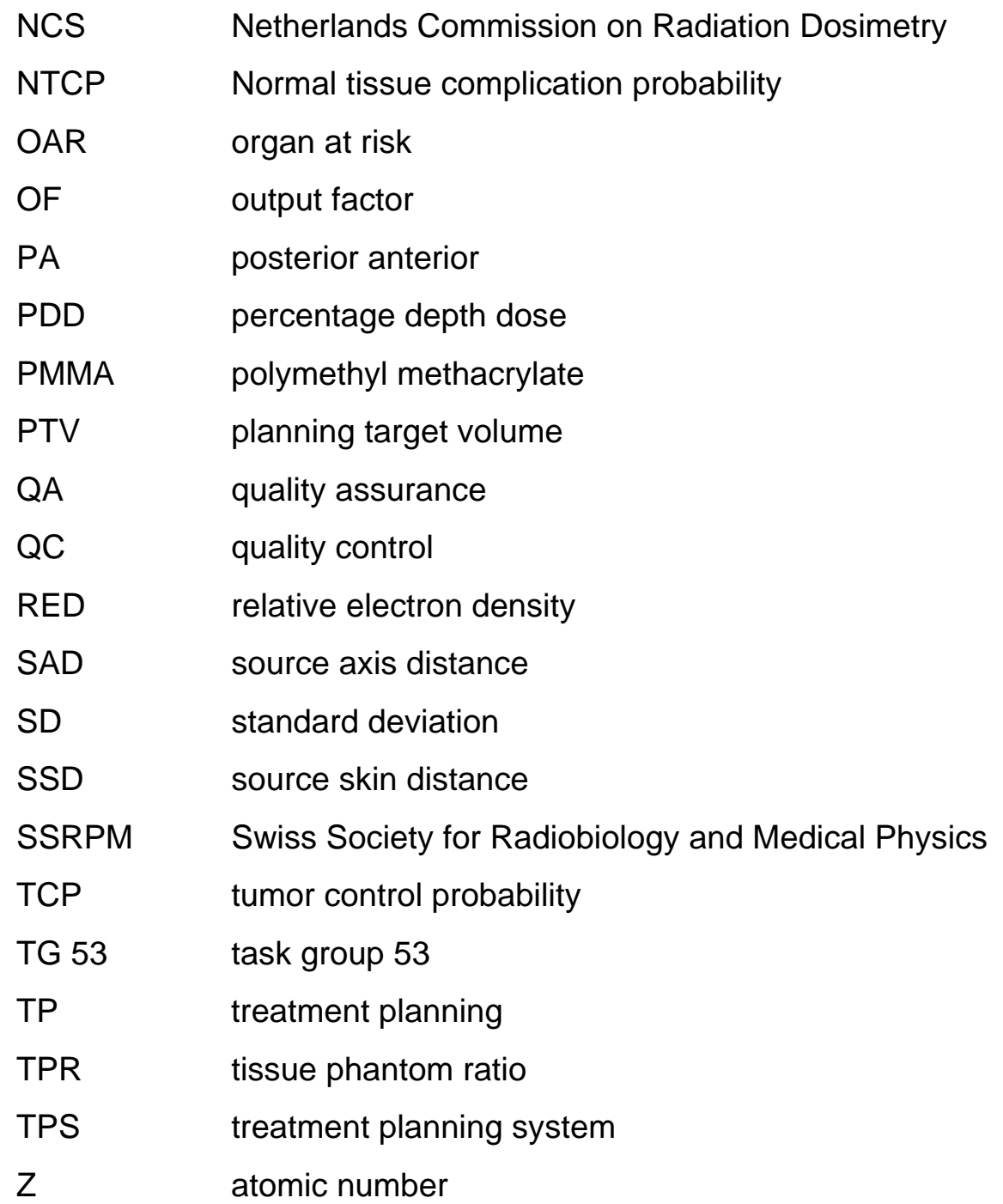




\section{Introduction}

At present many radiotherapy institutions have replaced their old "2-D" treatment planning system (TPS) by a modern "3-D" system. Such a 3-D system has a much greater functionality, therefore the procedures required for acceptance testing and commissioning (initial verification), and for periodic quality control are much more extensive and complex than with the 2-D systems.

In the past the main activities in the field of quality assurance (QA) of TPS concerned the verification of dose calculations. A number of authors reported the results of comparisons of measurements and calculations for a limited set of geometries (e.g. Westermann et al. [1], Rosenow et al. [2], Wittkämper et al. [3]). More recently AAPM Task Group 23 published a test package for QA of a TPS [4]. In general these tests only revealed the limitations of the dose calculation algorithms and of the beam data input. Other types of checks, for instance of non-dosimetric features of a TPS, such as tests to determine the accuracy in reproducing the geometric size of contours, tissue density or CT-numbers, have only been discussed by a few authors (e.g. McCullough and Holmes [5], Brahme et al. [6]).

More recently, a number of working groups in different countries have evaluated their specific national requirements on QA of a TPS and have drafted a document with recommendations.

\subsection{Review of activities}

\subsubsection{Early reports on QA of TPS}

In one of the earliest more general reports, from Canada (Van Dyk et al. [7]), detailed guidelines are given regarding sources of uncertainty, suggested tolerance levels, initial and repeated system checks. This report is therefore a useful document for physicists involved in QA of TPS. Another comprehensive report on this subject has been published by physicists in the United Kingdom, which concentrates primarily on requirements for commissioning and ongoing performance testing (IPEMB [8]). In a more recent report of that organisation the entire treatment planning process, including beam data acquisition, the commissioning of a TPS and the quality control of a treatment plan, have been outlined (IPEM [9]). Furthermore, recommendations for the quality control of a TPS for teletherapy have been published in Switzerland (SSRPM [10]). This report describes several tests and lists criteria for dose 
differences that are considered to be acceptable.

Nevertheless, these reports do not deal with the many QA issues that are important when the full range of tools of a 3-D TPS is employed. A more comprehensive approach to the QA of 3-D treatment planning has therefore been formulated by the American Association of Physicists in Medicine (AAPM) Task Group 53 (Fraass et al. [11]). In that report many issues related to the QA of a 3-D TPS such as QA of software, procedures, training as well as testing, documentation and characterisation of the non-dosimetric aspects of treatment planning are discussed. Basically the QA of the whole process of clinical use of a TPS throughout the entire treatment preparation has been described.

\subsubsection{Recent reports on QA of TPS}

The International Atomic Energy Agency (IAEA) has published an elaborate report describing a number of issues related to the QA of TPS [12]. The report can be considered as an extension of the AAPM TG 53 report with the emphasis on practical tests to be performed when operating a TPS in clinical practice. An accident that resulted in large overdoses (IAEA [13]) has been reported and the causes were related to errors in the use of a TPS and inadequate QA procedures. As a consequence, the IAEA started procedures for on-site review visits for QA in external radiotherapy planning. Both IAEA activities emphasize the need for users of a TPS to perform a thorough commissioning programme before applying their system in the clinic, as well as a programme of continuous QA during its clinical use.

Most recently the European Society of Therapeutic Radiology and Oncology (ESTRO) completed a document on a set of QA tests of a TPS [14]. In this QUASIMODO project a number of tests proposed in the current NCS report (see 1.1.3) have been evaluated, for instance with respect to the time involved in performing these tests. It was the aim of the QUASIMODO project to design a limited number of essential tests, which should be carried out before a treatment planning system is used clinically. In the final chapter in that booklet realistic examples are given to illustrate in more detail in which way these tests can be performed in practice. In an appendix a categorization has been made of the QA tests to be performed by an individual user, or by the vendor or a users group of a specific TPS. 


\subsubsection{The present NCS report}

In 1996 the Netherlands Commission on Radiation Dosimetry, NCS, installed a task group to formulate guidelines for the quality assurance of a 3-D TPS, in order to help the users in the design of their QA procedures. The task group consisted of users of various types of 3-D treatment planning systems with expertise in the QA of their specific systems. The members came from different institutions in the Netherlands, varying in size and availability of human resources. In the present NCS report the work of AAPM TG 53 was taken as the outline of the issues to be addressed. The TG 53 report provides the user with an excellent framework to design a QA program for the TPS used in his or her clinic. It treats extensively what has to be tested. The details of such a program, however, will depend on the specific type of system and its clinical utilisation, however. Thus each user will have to work out how each item has to be tested. The NCS report is intended to be complementary to the AAPM report and consists of examples of practical tests.

In March 2000 most chapters of the report had been written, but some parts still had to be filled in. Many colleagues in radiation therapy physics had repeatedly expressed the need for more information on the QA of 3-D TPS. Therefore the task group decided not to wait until all chapters were in final form, but made the material available on the NCS website (www.ncs-dos.nl) as a preliminary report and to be considered as work in progress. Feedback from various users was used to improve the report and between March 2000 and July 2005 new versions of the various chapters were written and missing sections were added.

\subsection{Aspects of QA of TPS}

\subsubsection{Categories of test items}

Comprehensive QA of a TPS covering all its aspects of use is a major enterprise and in general not a realistic goal for an individual user. It is therefore important to identify:

a. those items of which the test results depend exclusively on the properties of the system and software design;

b. the test results that are also dependent on the configuration process performed by the user at the time of commissioning;

c. results of tests that also depend on external data entered at the time of treatment 
planning (e.g., CT-data).

If data input by the user (cases b and c) is a factor of influence, the tests should certainly be performed by the individual user in order to verify correct implementation of that data or to determine the accuracy of performance of the TPS related to the specific data. In the other situation (case a) test results provided by the vendor or other users of the same type TPS (and software version) may be applied, and the user may decide not to test these items.

\subsubsection{Requirements for shared approaches}

In order to enable the user to take such decisions, in the first place the vendor should provide clear and complete documentation on the software and in particular the dependencies on commissioning data. Secondly, if test results of others are to be used, the specific design and execution of the tests, including all relevant parameters, should be clearly documented. For this purpose exchange of information and sharing of QA activities within a TPS user group is extremely useful.

Ideally a clear distinction should therefore be made between activities which may be performed by the vendor of a specific system, user groups of a TPS, and those tests which should be performed after the beam modelling process in that TPS of specific beams of a department. The vendor should provide a user with as much information as possible about the way an algorithm is implemented in its TPS. Also results of previous tests performed with that system should be distributed to its users. User groups could be very helpful in testing specific aspects of a TPS. For instance, the accuracy of a specific dose calculation algorithm at various beam energies can be assessed by a user group, while the individual user should test that algorithm for a local clinically applied treatment technique. Also non-dosimetric issues, for instance related to patient and beam description properties of the system, should not be tested by each individual user. In this report we will focus our attention on those tests that have to be performed by individual users. The separation between the various types of tests is, however, not always easy to make. Whenever possible this will be indicated in the text of this report.

\subsubsection{Evolution of QA of a TPS}

QA of a TPS is a dynamic process which evolves with time. Besides the use of a 3-D TPS to produce the customized treatment plan for a more or less routine technique, which may vary from single-plane planning of two rectangular fields to full 3-D dose calculations for multiple 
conformal fields, special software is often applied for specific treatment techniques. For instance, treatments with stereotactic fields or with intensity-modulated radiation therapy (IMRT) fields, lead to the introduction of new software or even specific TPSs for these applications. In this report we will not discuss the special tests to be performed on these systems, e.g., related to the use of very small fields or moving leaves of a multi leaf collimator (MLC). It may be expected that the experience currently being gained in a number of centres with respect to tests performed during clinical implementation of IMRT, will result in well-documented guidelines for QA of treatment planning of IMRT in the near future.

\subsection{Tolerances and accuracy}

\subsubsection{Accuracy of treatment}

Accuracy requirements for radiotherapy treatment should basically be derived from the radiobiological behaviour of tumour cells and normal tissues, as well as from clinical evidence, which represents this behaviour. The wide diversity in tumour sensitivities and normal tissue tolerances to radiation found in practice, could lead to a range of accuracy requirements. As this is considered to be undesirable in practice, requirements should reflect the most critical situations encountered in regular radiotherapy practice.

In a report published by the physicists in the United Kingdom (IPEM [9]) an overview is given of clinical evidence for the importance of radiotherapy accuracy. It is concluded that a difference in absorbed dose of $10 \%$ is detectable in tumour control, and that a difference of $7 \%$ in absorbed dose can be observed in normal tissue reactions. From an extensive review of dose-response data, Brahme et al. [6] concluded that the standard deviation (SD) in the mean dose in the target volume should be at most $3 \%$ to have a control of the treatment outcome with a $5 \%$ tolerance level. This is in agreement with a recommendation given by Mijnheer et al. [15] based on a review of steepness of dose-response curves observed for normal tissue complications, and other clinical observations. These authors concluded that transfer of clinical data between different institutions requires the dose to be known at the specification point within $7 \%$ accuracy, which they equated to 2 SD. An even stricter requirement of $2 \%$ has been suggested as a future goal in ICRU Report 24 [16], but such a requirement might not be realistic due to the limitations in our knowledge of physical parameters in the dose determination. 
Considerations of heterogeneity in dose and tumour cell characteristics over the target volume have been used as an argument for less strict dose requirements at other points in the target volume, leading to a $5 \%$ level (1 SD) over the entire target volume (IPEM [9]). However, a higher accuracy will be needed to obtain more accurate tumour control data.

Due to the limited availability of clinical data, an accuracy criterion of $4 \mathrm{~mm}$ (1 SD) in the position of beam edges has been formulated (IPEM [9]). Although considerations of practical feasibility have guided the numerical value of this criterion, it falls well within the range of set-up errors as observed during portal imaging studies (Hurkmans et al. [17]). It should be noted that for specific treatment situations, such as the junction of fields or during (dynamic) intensity-modulated radiotherapy, the position of field edges has to be known with much higher accuracy .

\subsubsection{Tolerances in treatment planning}

If an overall accuracy in the value of the dose delivered to the ICRU specification point of the order of 3 to $4 \%$ is required, then only a small margin is left for the accuracy of the dose calculation part of the total procedure. This is because the uncertainties in the other parts of the dosimetry chain, such as calibration of the beam under clinical conditions, and treatment delivery, have relatively large uncertainties. In the past generally rather simple recommendations were given for the accuracy of dose calculations such as $2 \%$ or $2 \mathrm{~mm}$ in regions of the beam with small or large dose gradients, respectively. More recently the complexity of the beam and patient geometry was also taken into account in the requirements for the dose calculation accuracy. The exact recommendations differ, however, in the various reports as summarized in a review by Venselaar et al. [18]. These authors defined a consistent set of tolerances for the accuracy of dose calculations in photon beams. Their approach has been followed in this report, and has been elucidated, both for photon and electron beams, in Appendix A.4.1. It should be noted that due to practical issues wider criteria for more complex calculations may be accepted for the time being, but should ultimately converge to criteria based on the radiobiological and clinical considerations mentioned before. 


\subsection{Definitions}

Because a number of concepts that are used in this report might be interpreted in different ways, definitions are given here of the most common items encountered in QA of TPS.

Radiotherapy treatment planning (TP) is the entire process to prepare the radiation treatment of a cancer patient. This process includes imaging studies, definition of target volumes, design and optimisation of the irradiation technique, evaluation of the treatment plan and implementation of the plan on the treatment unit.

In the treatment planning system (TPS) the patient data are entered, the anatomy is defined, beams are set up, the dose distribution is calculated, the plan is evaluated in terms of dose, volume (and biological effect) and output is prepared for documentation and for transfer of data to block cutter, simulator or treatment machine. The TPS consists of a software package or a combination of different packages and its hardware platform. This can range from a single stand-alone computer to an entire network of many workstations and of various peripheral devices.

A three-dimensional treatment planning system (3-D TPS) refers to a modern TPS (hardware and software) that offers the functionality of: $a$. constructing a 3-D patient model, based on a volumetric CT-scan; $b$. simulating 3-D configurations of beams, i.e. with arbitrary beam orientations, isocentre position and field shapes; c. performing 3-D dose calculations, i.e. with an algorithm that takes the 3-D aspects of patient, beams and interaction physics into account; $d$. evaluating and optimising 3-D dose distributions, using tools like dose-volumehistograms, Normal Tissue Complication Probability (NTCP) calculations and optimisation algorithms; e. advanced viewing of patient anatomy, treatment beams and dose distributions in their 3-D relationships.

Quality assurance (QA) of a 3-D TPS is the total of procedures that are carried out to determine the quality (in terms of accuracy and reliability) of the TPS and to guarantee that the system performs according to previously established specifications. One should distinguish the procedures of acceptance testing and commissioning (initial verification) and periodic quality control (ongoing verification). 
Acceptance testing is the procedure to confirm that the TPS performs according to its specifications as documented at the moment of purchase. These specifications are dictated by the functionality implemented in the system and by the quality of the algorithms and should be defined by the manufacturer. In the strict sense this procedure should be called product acceptance testing.

Commissioning is the procedure required to bring the new TPS or new software release into safe clinical operation. The clinical user should define the contents of this procedure. Commissioning includes introducing geometric and dosimetric data into the system to define the treatment machine and its beams, performing tests to verify correct functioning of the entire software and to determine the limits of accuracy of the various calculations. For completeness establishing QC procedures and training of personnel should be also be included.

These procedures could be called clinical acceptance testing in contradistinction to the previous procedure which we indicated by product acceptance testing.

Periodic quality control $(Q C)$ is the procedure to verify periodically the correct functioning of the TPS. QC tests are repeated with a pre-set frequency; some may be carried out automatically, others manually. QC has therefore a more restricted meaning than QA which includes also other aspects related to proper use of a TPS, such as user training to ensure that the TPS is used correctly.

A treatment planner is a person who is using the TPS at some moment in order to produce a plan for clinical radiation treatment of a patient. This can be either a medical radiation dosimetrist, a radiation therapy technologist, a clinical radiation physicist or a radiation oncologist.

The (TPS) user is the person responsible for the correct functioning of the TPS in clinical practice and thus for the quality assurance of the TPS. In most cases this will be a radiation oncology physicist, registered as clinical or medical physicist. QA testing of the TPS will be carried out by this person or by personnel that he or she supervises. 


\subsection{Contents of this report}

\subsubsection{Aims and scope}

The primary aim of this report is to provide practical procedures for the initial QA of a 3-D TPS for external photon and electron beam treatment planning, which can be applied to a large variety of TPSs. The emphasis is placed on the commissioning of a TPS, some guidelines for acceptance testing and periodic quality control are also given. This report may be considered as an extension of the AAPM TG53 report and consists basically of tests that cover the recommendations of the AAPM TG53 report to a great extent, but certainly not completely. No claim of completeness is made, as this would be an unrealistic goal. In order to be practical, the descriptions of the tests are as specific as possible, without going into unnecessary detail. In some cases a more detailed description of a test could only be designed with reference to a specific type of TPS. In these situations a more global indication of the test was preferred.

The current report is limited to the QA aspects of the treatment planning system. This concerns verification of proper functioning of the TPS as well as establishing correct use of the TPS for the required accuracy. Such a QA programme comprises the computer hardware and software, but also the dosimetric and anatomical input data and the output of the system. Furthermore, the subject of the report is restricted to conventional treatment planning, i.e. forward treatment planning for single-segment conformal radiation therapy. The specific aspects of QA for inverse treatment planning, mathematical optimisation, multisegments treatments and intensity modulated radiation therapy are not dealt with here.

In this report we implicitly assume that all functionality is implemented in one single system, but a clinic may also use a combination of systems. Virtual simulation software on a CT-Sim system is often used for contouring of structures and maybe also for beam set-up; a TPS is then only used for calculation and display of dose distributions. Procedures for the QA of virtual simulation software are not given explicitly in this report.

Finally, we did not include obvious checks, like whether an entered number is read and processed correctly by the TPS in the suggested tests. The same applies to items that are general for any computer hardware and software system. 


\subsubsection{Outline of chapters}

In this first chapter general aspects of TPS QA, of published information and this report have been discussed. Chapters 2 to 5 each deal with a specific step in the treatment planning process: definition of the patient anatomy, definition and setting up of the beams, calculation of dose distributions and evaluation of the treatment plan. Chapter 6 addresses plan documentation and data export to an accelerator, while chapters 7 and 8 treat the general items of periodic quality control and system management. QA of a TPS does not only concern the separate steps, but also the mutual relations. In each chapter the issue of correspondence with the results of the previous step is addressed, while more general aspects of transfer of data are discussed in Chapter 8.

In research applications, dose calculations might be performed outside the TPS with separate software. In such a configuration the correct transfer of data from one system to another and bookkeeping issues require extra attention. These issues are discussed, to a limited extent, in Chapter 8 of this report.

\subsubsection{Format and phrasing}

In each subsection of this report a set of suggested tests is presented, preceded by a clear statement of the scope of the test and a short explanatory background. For more background information the reader is referred to the corresponding sections in other more detailed reports such as the AAPM TG53 and IAEA reports.

In most cases the test descriptions are phrased in the imperative mood, like "Check ...", "Test ..." or "Verify ...". These are meant to be recommendations and should not be taken in a compulsory sense; the authors do not claim any authority exceeding that of their professional expertise. In some cases phrasings like "One should check ..." or " ... should be verified" are used. Then a statement of more general validity is intended; it is the opinion of the authors that "good QA practice" requires such an action, but the necessity of implementation is left to the judgement of the user. 


\section{Anatomical description}

Treatment planning is based on anatomical characteristics of the individual patient. These include the external geometry, the localization and extent of the tumour and organs at risk as well as variation in the tissue density. These characteristics constitute an anatomical description or anatomical model. Such a description is derived, preferably, from a set of CT images, but a set of patient contours in combination with simulator images may also be used. On the basis of this data set anatomical structures are defined. These are geometrical elements, such as points, contours and volumes that specify the patient anatomy in the TPS. Each patient file or patient database entry in the TPS needs to be uniquely linked to one or more anatomical descriptions, and each anatomical description to one or more beam arrangements.

In this chapter tests are presented to ensure that the anatomical data are correctly linked to the specific patient (section 2.1), that the input of such data, images or contours (section 2.2) is correct. In section 2.2 also the conversion of CT numbers into relative electron densities is discussed, tests are described to verify that images resulting from operations on the original data are correct and finally the use of images other than CT-data is addressed. In section 2.3 tests for correct generation of structures, contours and volumes are presented.

\subsection{Basic patient entry}

\section{Scope}

To ensure that the data constituting an anatomical description are linked to the proper patient file or patient database entry in the TPS.

\section{Background}

Special attention has to be paid to those stages in the treatment planning process where there is a chance to provide a patient file or database entry with wrong input data, either from another stage in the treatment planning process or even from another patient. Such errors are most likely to happen during input and retrieval of patient data.

The infrastructure used to provide the specific TPS with data, the internal organization of the TPS and its system management determine which errors may occur. The following tests are given as examples. 


\section{Suggested tests}

a. Uniqueness of patient

Enter two cases (patients) with identical names and other relevant identification fields and verify that the TPS gives appropriate warnings.

b. Association with existing patient

Enter a second anatomical description for an existing patient. The TPS should warn for overwrite and/or association of a new description to an existing case.

c. Retrieving patient data

Create and store a patient with a number of different anatomical descriptions. Retrieve the case and verify that the correct descriptions are retrieved.

\subsection{Image conversion, input and use}

Patient geometry for treatment planning can be derived from two types of data: either images from tomographic devices such as a CT-scanner adapted to radiotherapy requirements or a simulator with CT-extension, or contours from any type of body contouring device. From a treatment planning point of view, commissioning of the latter type (section 2.2.2) is merely a subset of the first type (section 2.2.1) and will be treated as such. Planar images that are reconstructed from sets of tomographic images will be described in section 2.2.3. Use of other images such as MRI, SPECT or PET within a TPS and the registration (matching) of various image sets, such as MRI-CT, is still under development and therefore only briefly addressed in sections 2.2.4 and 2.2.5.

\subsubsection{Image input}

\section{Scope}

To ensure that CT-scan data are imported correctly in the TPS. This concerns the integrity and the completeness of CT image input. Most of the tests are also applicable, with appropriate adaptations, to other tomographic modalities like MRI.

\section{Background}

CT-scan data contains besides images also various image related parameters. Image integrity depends on a subset of parameters that are vital for the correct interpretation of the images by the TPS (e.g., patient orientation and slice position). Completeness of image data requires that all additional essential information is transported to and entered into the TPS correctly. Examples are text comments or contours made at the CT-scanner. Depending on 
the development of e.g. the DICOM and DICOM-RT standard, other items may be defined prior to introduction in the TPS and should then be included in appropriate tests. Incompleteness of input data may occur when a TPS uses some time parameter to sort incoming images, as file times can be influenced by network load or simultaneous input from other sources and images may be lost.

The format in which the parameters are stored is critical to correct interpretation by the TPS and generally depends strongly on the type of CT-scanner and software release, unless the manufacturer adopts a standard, such as DICOM. The integrity and completeness should be tested for the specific CT-scanner and accompanying software release during commissioning of the TPS, after any change in the equipment of the CT-scanner or after changes in the CT interface of the TPS.

For most of the following tests the type of phantom is not critical, as long as the relevant parameters are well-defined. The integrity of the infrastructure used to provide the TPS with data is discussed in chapter 8.

\section{Suggested tests}

Integrity of data

a. Consistency of scans

Check that the TPS handles all patient data that belong together as one set and rejects all inconsistent data. Generate erroneous data sets, either at the CT-scanner or at an intermediate location (network directory) between the scanner and the TPS. Send the following erroneous data sets to the TPS:

- A data set with duplicated slice. This corresponds to a selection error at the CTscanner. The TPS should detect the extra slice and give a warning.

- Two data sets with intermixed file time attributes. Check that both data sets in the TPS are correct and complete. If a TPS uses another parameter to sort incoming images, its sensitivity to realistic changes in that parameter should be investigated.

- A data set with CT FOV changes. The TPS should either handle the different scales correctly or detect images which a different FOV in one data set and give a warning.

b. Scan parameters

Generate data sets with various slice thicknesses, indices and off-sets. Suggested thickness and index combinations (mm): 5/5, 2/5 and 5/2. Suggested offset ( $X, Y$ in $\mathrm{mm}$ ): $(0,0)$ and $(100,100)$. Verify that the TPS recognizes these parameters. If the TPS does not directly display the internally used values, the actual value of these parameters in the TPS might be derived from other TPS parameters, e.g. from the appearance of reconstructed planes or DRRs. Suggestion: send the same scans from the scanner to an independent DICOM viewer and check the parameters. Do the same following export 
of these scans from the TPS to see which parameters 'survived' the TPS.

C. Patient orientation

Make CT scans of a phantom with various clinically used orientations, such as headfirst, feet first, prone, supine, left side and right side. As an example we propose a socalled ULF-phantom. Such a phantom consists of polystyrene bars and shows a capital $U, L$ and $F$, when the viewer faces the Upper, Left and Feet side, respectively (fig 2.1). Perform some of the scans with other fields-of-view used clinically. Check the representation of the patient orientation in all data sets, in axial slices as well as scanograms (topograms, scout views). If the TPS has an orientation aid, this tool can be helpful in the tests, but correspondence in orientation to the original images should also be checked.

Fig. 2.2 shows an axial slice of the ULF phantom scanned in supine head-first position as seen from caudal, i.e., the most common orientation. Note the correspondence of the displayed phantom and the orientation aid.

d. Geometry of slices

Scan a phantom of well-defined dimensions, (e.g., a block of polystyrene or solid water used for dosimetry purposes) and check its dimensions in three directions in the TPS using a central FOV and a FOV with $100 \mathrm{~mm}$ offset in both $X$ and $Y$ direction. (detailed geometry tests are given in sections 2.3.2 and 2.3.3).

In addition markers may be fixed to the radiotherapy table top insert, which will show up in all patients with adequate FOV. This can also be helpful in periodic QC.

e. CT number representation

Check the representation of CT numbers (HU values). This can be done by scanning a phantom with known composition and electron density, e.g., the RMI 465 phantom. See appendix A.2.1 for detailed suggestions.

\section{Completeness}

g. Text information

Check that text information, e.g., comments, scan date or device name, relevant to treatment planning is available in the TPS, especially in the initial stage when the patient model is constructed

h. Check that all parameters that are used inside the TPS are read. Examples are tube voltage, if used for CT number to electron density conversion, slice thickness, if used in generation of digitally reconstructed radiographs (DRRs), or points or contours entered at the CT scanner if used as an aid to define target volume or treatment technique. 


\subsubsection{Contour input}

Scope

To ensure that data generated by a contouring device are transferred correctly into the TPS.

\section{Background}

A set of one or more external contours is the minimum data to construct an anatomical description of a patient. Such external contours are acquired with some kind of body contouring device. Based on measurements in projection radiographic images the position of internal organs like lung and spinal cord may be estimated. These contours are entered via a digitizer, film scanner or file transfer into the TPS. The first point of concern is the correctness of the contours itself. Furthermore, the TPS might construct interpolated contours in between imported contours. The correctness of this interpolation should be checked, especially in regions with large contour variations, e.g. in the thorax-neck transition region.

The tests in this section should be adapted to the errors that may be expected for the specific type of input device used. A digitizer might cause position dependent errors over its entire active area. Linear deviations in either scan or transport direction may occur with a film scanner. With file transfer, file integrity is a point of concern; tests have been described in section 2.2.1.

\section{Suggested tests}

a. Contour input

Generate contours in multiple slices of a phantom with scale factors and orientations representative for clinical use, see e.g. fig. 2.3. Transfer these contours into the TPS by standard pathway (digitizer, film scanner or file transfer) and verify the dimensions of the phantom represented by the TPS. This is a combined test of the contouring device, data transfer and TPS input.

b. Interpolation

Verify the interpolation of external contours in between original slices. Methods to do this are inspection of sagittal and coronal reconstructed images and 3-D display See for further details section 2.3.5.

c. Digitizer input

Enter the contours of fig. 2.4 in the TPS. Check the position of all corner points. Digitizer input requires testing of the entire active area.

d. Film scanner input

Enter fig. 2.5 in the TPS and check the dimensions along the scan direction and at both 
sides along the transport direction. Film input requires a linearity check along both main axes including a check of parallel transport.

\subsubsection{Image reconstruction}

\section{Scope}

To ensure that images reconstructed from any set of images (CT, MRI , etc.) are represented in the TPS with correct geometry and orientation. To assure correctness of grey-scale representation of scanned and reconstructed 2-D images. To establish appropriate scan parameter settings for required accuracy. Digitally reconstructed radiographs are not treated here, but in section 3.3.

\section{Background}

Grey-scale images may be reconstructed images in sagittal, coronal or oblique planes. They are used to identify anatomical structures, verify beam positions or to evaluate dose distributions. They should thus have correct geometry, orientation and grey-scale values. The accuracy of the reconstructed geometry, however, will depend on the slice distance and thickness of the original CT-scan and the variation of grey-scale values in the original geometry. Tests of the reconstruction algorithm should therefore be carried out with minimum slice spacing and similar thickness; for clinical application the accuracy of reconstructed images obtained with larger values of the scan parameters should be investigated for each anatomical site. The grey-scale values in the original and reconstructed images will depend on the specific scan protocol; but should be the same at identical positions in the patient (model).

\section{Suggested tests}

a. Geometry of test images.

Scan a phantom with well defined, relatively simple, geometry, e.g. the ULF phantom of fig. 2.1 or the abdomen phantom of appendix A.2.2 in standard head-first orientation. Use minimum spacing and thickness (e.g. $2 \mathrm{~mm} / 2 \mathrm{~mm}$ ). Reconstruct various sagittal, coronal and oblique images and compare the geometry with expected (calculated) results. Repeat the procedure for larger scan parameter settings and determine the accuracy of reconstruction.

b. Geometry of clinical images

Scan (parts of) an anthropomorphic phantom (e.g. Anderson Rando phantom) in standard head-first position, with minimum slice spacing and thickness and with clinically used scan parameter settings. Also scan each part of the phantom in sagittal, coronal 
and oblique orientations with minimum scan parameter values. Reconstruct various sagittal, coronal and oblique images from the standard scan data and compare the results with the corresponding scans.

Fig. 2.6a shows a reconstructed central sagittal slice of the Rando head phantom scanned in standard orientation; fig $2.6 \mathrm{~b}$ shows the same slice directly scanned in sagittal orientation. Slice spacing and thickness were $2 \mathrm{~mm} / 2 \mathrm{~mm}$.

c. Orientation of images.

Check if the reconstructed images in test a. have correct orientation. Display of beam projections with specific gantry and table angles can be helpful.

d. Grey-scale representation

Compare a CT image of the abdomen phantom stored (screen capture) at the CTscanner with the same image displayed on the TPS using identical level and window settings. Compare the reconstructed images with the original axial scan images. Greyscale values at similar positions in the phantom should be identical. Instead of the abdomen phantom a slightly tilted PMMA or polystyrene slab may also be used: the partial volume effect will lead to the desired gradient of grey-levels.

\subsubsection{Specific requirements for MR images}

\section{Scope}

To ensure that MR images used in a TPS complementary to CT images, provide a correct geometric representation of any structure of interest.

\section{Background}

MR images provide a soft tissue representation that is often superior to that of CT images. Therefore MR images are valuable aids in target definition for radiotherapy treatment planning. In contrast to CT images, MR images lack electron density information used for dose calculation, and they suffer from geometric distortion, especially near the periphery of the patient. Therefore no direct use of MRI has been made thus far in treatment planning, but anatomical structures are identified on a MR data set and transferred via image registration (section 2.2.5) to the CT data set that is used as a basis for treatment planning. This complementary use of MR images is addressed in this section and only the image aspects that are most critical to radiotherapy use: the geometric representation of anatomical structures. More extensive tests are described in IPEM report 81 [9]. Proper selection of scan sequence, band width and correction procedures, if available, may minimize distortion. Some residual image deformation is often unavoidable, and quantification of this deformation is then the only option. 


\section{Suggested tests}

a. Test phantom

Define a phantom with dimensions and composition representative for each anatomical region for which images have to be acquired. Suggestions for phantoms are given in IPEM report 81 . Simple alternatives are a cylinder $(\varnothing 20 \mathrm{~cm}$, length $20 \mathrm{~cm})$ to simulate a head and the body shape of fig. 2.7 to represent a torso. Phantoms are normally made of PMMA and filled with a solution of copper sulphate, nickel chloride or manganese chloride (IPEM [9], AAPM [19]). A grid of PMMA rods facilitates the assessment of image distortions. Rods that run at $\pm 45^{\circ}$ in axial, coronal and sagittal planes are used to check slice position.

b. Geometric representation

Scan the phantom in an MR scanner with a scan sequence and band width corresponding to the intended clinical use. Carefully align the phantom with the scanner's main axes. Transfer the axial images to the TPS and check the position of the internal markers over the entire phantom to quantify any distortion.

If sagittal or coronal images are also used for treatment planning, then the procedure should be repeated twice with a 90 degree rotated phantom.

c. Slice position

In the same scan measure the centre of the crossed (' $X$ ') rods in all axial slices. The increase (or decrease) in distance between the corresponding centres should be twice the difference in slice position.

\subsubsection{Image registration}

\section{Scope}

To ensure that MR images that are registered with $\mathrm{CT}$ images provide a reliable geometric representation of any structure of interest.

\section{Background}

Image registration, the definition of a transformation in positions between two image data sets, is a rapidly expanding area of research, to which many problems are linked that are not yet solved. A review of current registration methods has recently been published (Hill [20]). While still under development, image registration is increasingly being used clinically, because of its high potential to help improve target definition. A pragmatic justification for that practice is that any reasonably accurate transformation is definitely an improvement as compared to 'mental image registration' based on interpretation of images that are merely displayed on a light box. However, because the inaccuracies involved in image registration 
are not always clearly visible (i.e. the errors due to chemical shift) a false sense of accuracy may result. Therefore QA phantom tests and clinical tests are necessary to estimate the reliability of the registration.

Several factors prohibit an a priori quantification of the accuracy of image registration. The outcome of a registration process is inherently dependent on the difference in information content of two image sets. Furthermore, the image process may have been altered by the presence of a patient in the imaging device (e.g. MRI). Moreover, the 'true' transformation is usually unknown in clinical cases. Therefore QA tests with phantoms can help to gain confidence in, but not to guarantee correctness of, an image registration procedure. The accuracy of image registration should thus be assessed for each clinical case, preferably by using redundant information in the images.

The suggested tests in this report are confined to MR-CT registration, and to rigid body transformations. The thus defined transformation of MR images might either be used to reslice these images or to transfer any anatomical structures defined on MRI to the reference data set (CT).

\section{Suggested tests}

a. Phantom checks

Create T1 and T2 weighted MR data sets of the phantoms described in the previous section. The scan sequences and band widths used should be those of the corresponding clinical scan protocols. Perform a registration of these data sets to a CT data set of the same phantom. Quantify the discrepancies found in the positions of the centres of the rods.

b. Clinical checks

In any MR study that is to be used for image registration, two or more data sets should be obtained, which differ in particular in the representation of features that are to be used for image registration. E.g. T1, T2 and dark fluid scans might be obtained. Take care that no patient motion occurs in between the various scans (fixation). Use each MR data set for an independent registration with the CT reference set. The correspondence of the distinct transformations can be considered as an indication for the reliability of the registration. 


\subsection{Anatomical structures}

We assume the 3-D patient model is constructed on the basis of CT-scan data. For beam set-up and dose calculation purposes, a body outline and a planning target volume (PTV) may be required. Field shaping in beam's eye view (BEV) and dose-volume histogram (DVH) calculations may also require organs at risk (OAR) to be defined. Bulk inhomogeneity corrections require delineation of specific organs. The various delineated volumes are called anatomical structures here.

Other sets of imaging data may also be used in the TP process and structures may be defined on different data sets. The registration of various data sets and the transfer of structure information from one set to another are an important item. This section addresses the QA of defining anatomical structures, of generating and processing 2-D contours and of the construction of 3-D volumes by the TPS.

\subsubsection{Definition of anatomical structures}

\section{Scope}

To ensure that anatomical structures are defined uniquely and correctly and that any changes made by the user are monitored by the TPS.

\section{Background}

There should be no possibility of confusion to which patient and data set a anatomical structure is related. The properties of the anatomical structure and which contours belong to the structure should also always be clearly defined. It should not be left to the TPS user to realise that a change made in a structure may affect further steps in the treatment planning process; the TPS should keep track of that.

\section{Suggested tests}

a. Unique identification

Check how an anatomical structure is related to the specific patient (depends on database and file system) and whether there is a risk of confusion. If a new structure is defined with a name that already exists for another structure, what warnings are given? Can the data set of origin of a structure be identified? What happens if identical names for two structures defined on different data sets are used?

b. Unique properties

If the TPS distinguishes different types of anatomical structures (e.g. external surface, target volume, inhomogeneity) the structure type should be clearly defined and for one 
data set only one external surface can exist. Check the TPS response to the attempt to define a second structure with a different name, but also with the property external.

c. Structure contours

Check how 2-D contours, drawn in consecutive slices to define a structure, are related to the specific structure and whether there is a risk of confusion. Check how two separate contours, drawn in one slice to define the same structure, are handled.

d. Display modes

The mode of display and the colour of the structure should be consistent at any point in the TP process.

e. Maximum numbers

If there is a maximum number of anatomical structures per patient that may be reached in practice, the response of the system should be tested. The same applies for a maximum number of contours per structure.

\subsubsection{Automated contouring}

\section{Scope}

To test the accuracy of the segmentation algorithm that generates contours and to establish the optimum parameter settings.

\section{Background}

Each TPS has its specific algorithm to trace regions in a CT-slice with CT numbers within specified limits and to construct the encompassing contour. One should expect the algorithm to be able to handle the external surface, lung and bone tissues; smaller CT-number gradients may also be dealt with. For the specific type of CT-scan data, defined by scanner and scan protocol, and conversion method used, the optimum CT-number limits for each type of anatomical structure have to be derived in order to obtain the correct contours of the structure.

\section{Suggested tests}

\section{a. CT-scan geometry}

Make CT-scans of a test phantom with well-defined geometry and containing materials similar to lung, bone and soft tissue, e.g. the abdomen phantom of appendix A.2.2, with the different clinically applied scan protocols. Let the TPS generate the external, lung and bone contours and make a hardcopy plot of the axial slice through the centre of the structures (no partial volume effects). Compare the plot to a graph with the exact contours of the phantom. The two sets of contours should coincide within $1 \mathrm{~mm}$. 
b. Optimum scan parameters

Repeat the test with various settings of the segmentation algorithm parameters and investigate for each structure and scan protocol which settings yield the highest accuracy.

c. Maximum numbers

If there is a maximum number of contours per CT-slice that may be reached in practice, the response of the system should be tested.

\subsubsection{Manual contouring}

\section{Scope}

To test the accuracy of manual outlining tools that may be used to generate contours and to establish the optimum parameter settings.

\section{Background}

Target volumes will mostly be outlined manually (by mouse) and other anatomical structures for which automated contouring (in all or some slices) fails. The accuracy of the generated contour will depend on the skills of the user, but also on the level and window $(L, W)$ settings and on the size of the displayed image. For each type of structure the optimum L,W settings should be derived empirically and the range of appropriate display sizes (screen layout and zoom factors) should be established.

\section{Suggested tests}

a. Correct geometry

Use the CT-scan of the abdomen test phantom with well-defined geometry and contour the lung and the bone cylinder manually. Make a hardcopy plot and compare to the exact graph; the original contour and the average position of the manual contour should coincide within $1 \mathrm{~mm}$.

b. Contouring direction

If contours may be entered both clockwise (CW) and counter clockwise (CCW), enter the same contour in both directions and verify on hardcopy plots that the results are identical. Check that the computed volumes do not depend on whether the contours are entered CW or CCW, also when mixed (see section 2.3.5)

c. Optimum parameters

Repeat the test with various $(L, W)$ settings and investigate for each structure which settings yield the highest accuracy. Repeat the test for one structure on images with various display sizes and establish what screen layouts and zoom factors may be used. 
d. Maximum numbers

If there is a maximum number of points per contour that may be reached in practice, the response of the system should be tested.

e. Add margin to contour

The option to draw a contour with a predefined margin should be verified with the same phantom study. Note that this margin is defined in the plane that contains the contour (2D expansion), and thus a consecutive set of such contours will yield a different volume than will be obtained by a 3-D volume expansion (see section 2.3.5).

f. Distances

There will be a tool to read out co-ordinates of a point and to measure distances or angles by placing a cursor on the screen. Verify the correct functioning of these options with the phantom test.

\subsubsection{Manipulation of contours}

\section{Scope}

To ensure that all operations performed on contours yield correct results.

\section{Background}

The TPS will offer various tools to alter, copy or delete contours; the results of these operations and correct bookkeeping should be verified.

\section{Suggested tests}

a. Correcting

Edit contours obtained in a well-defined way. Change, delete and add points to the contours. Plot the result and verify that the new contour is correct. Store the new contours, then retrieve, plot and verify again.

b. Adding and deleting

Add a number of contours of one structure in various slices. Store the structure, then retrieve and verify that the set of contours is correct. Delete a number of contours of one structure in various slices. Store the structure, then retrieve and verify that the set of contours is correct.

c. Copying

Copy a contour from one slice to another within the same data set. Copy a contour from one data set to another. Verify the results.

d. Validation

Any change made in the contours of a structure should be detected by the TPS. Check if 
an existing 3-D surface of that structure is invalidated and preferably recalculated automatically.

\subsubsection{Construction of volumes}

\section{Scope}

To ensure correct computation of volumes, their 3-D surfaces and contours derived from these surfaces.

\section{Background}

On the basis of the consecutive set of 2-D contours in axial planes (CT-slices), the 3-D surface of the structure is computed. From this surface, 2-D contours may be constructed in sagittal, coronal and arbitrary planes of the same data set that intersect the structure. From this surface also 2-D contours may be constructed in planes of another data set. The structure may be expanded in 3-D with one predefined margin (isotropically) or different margins (anisotropically). One should verify that this expansion is truly $3-D$, i.e. margins are added along vectors perpendicular to the surface of the structure. These surfaces and contours are used to define anatomical structures, shape treatment fields, verify beam positions and evaluate dose distributions. The accuracy of the constructed surfaces and contours, however, will depend on the type of algorithms used, the spacing of the slices containing the original axial contours and the variation of shape in the original geometry. Tests of the reconstruction algorithm should therefore be carried out with minimum slice spacing; for clinical application the accuracy of reconstructed surfaces and contours obtained with larger slice spacings should be investigated for each anatomical site.

\section{Suggested tests}

a. 3-D surface computation

Use the CT-scanned abdomen test phantom and let the TPS compute surfaces from the derived contours. Check the correct shape of the surfaces in a qualitative way in 3-D display under various angles of view. Let the TPS compute the volumes inside the structure's surfaces and compare with the exact values considering the position of the first and the last slice through the phantom. If there is no separate option to compute volumes, the module to compute dose-volume-histograms may be used.

b. Non-regular spacing

Construct surfaces from contours in planes that are not regularly spaced. Therefore delete a number of contours of an original regular set, generate the surface and compare to the original surface. 
c. Non-axial contours

If the system allows surfaces to be constructed from contours in planes other than axial, repeat test $a$. for those orientations. Verify the consistency of surfaces constructed from sets of contours from planes with different orientations.

d. 2-D contour extraction

Construct the contours in a sagittal, coronal and two 45 degree oblique planes, make hardcopy plots and check the dimensions of the contours.

e. 2-D contour transfer

Construct the contours in various planes of another data set, make hardcopy plots and check the dimensions of the contours

f. Structure expansion

Expand one of the test phantom structures with a single margin and check the correct shape of the surface of the expanded structure in a qualitative way in 3-D display under various angles of view. Extract contours from the original and the expanded surface in various planes and measure the margins in those planes. Another approach to test 3-D expansion is given in appendix A.2.3

g. Copy structure

Construct a new structure as a copy of an existing one. Compare the surfaces of the new and the original structure in a qualitative way in 3-D display under various angles of view. Extract contours from the new and the original surface in various planes and compare. 


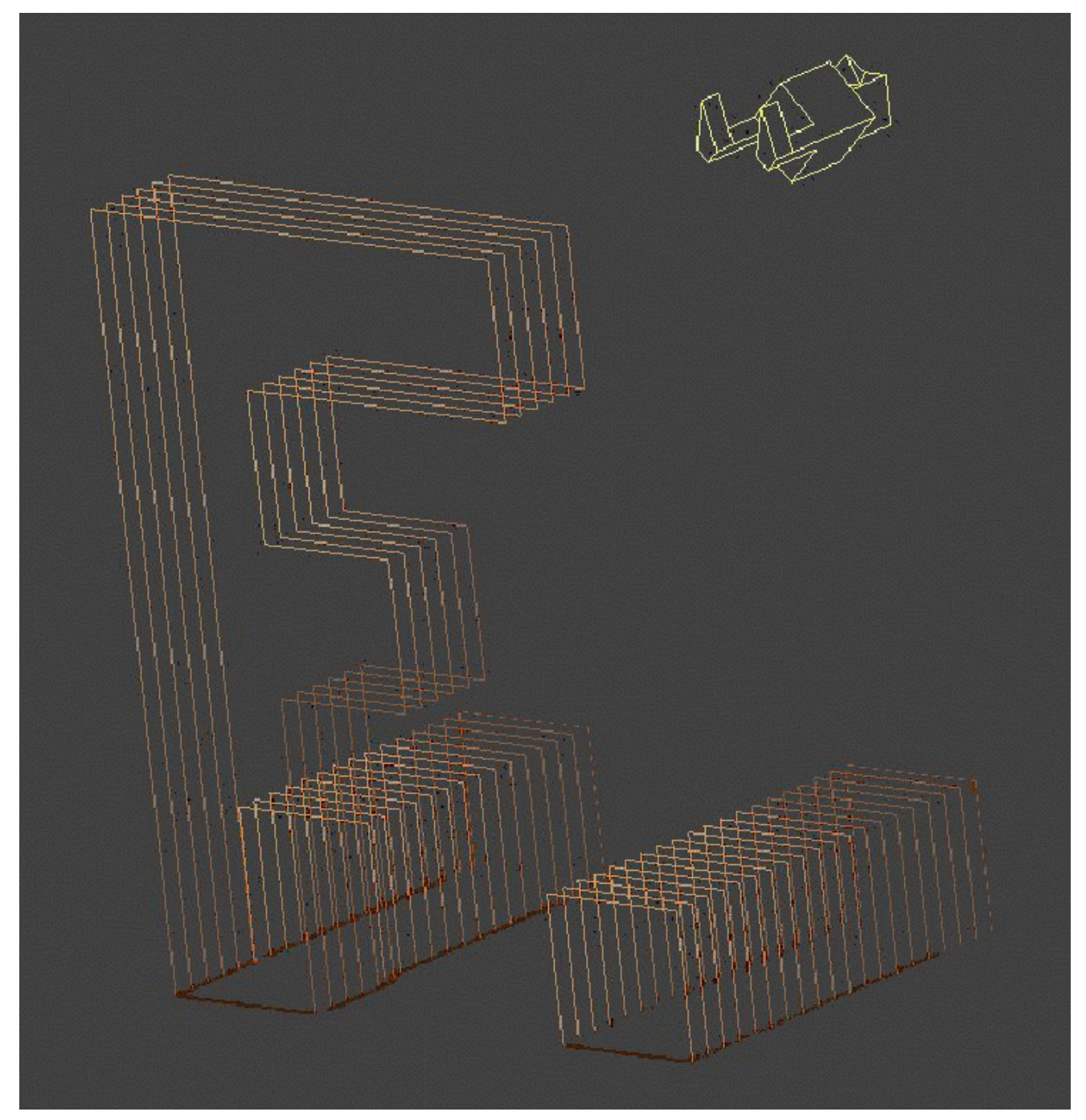

Fig. 2.1 ULF phantom used for checks of patient orientation. 


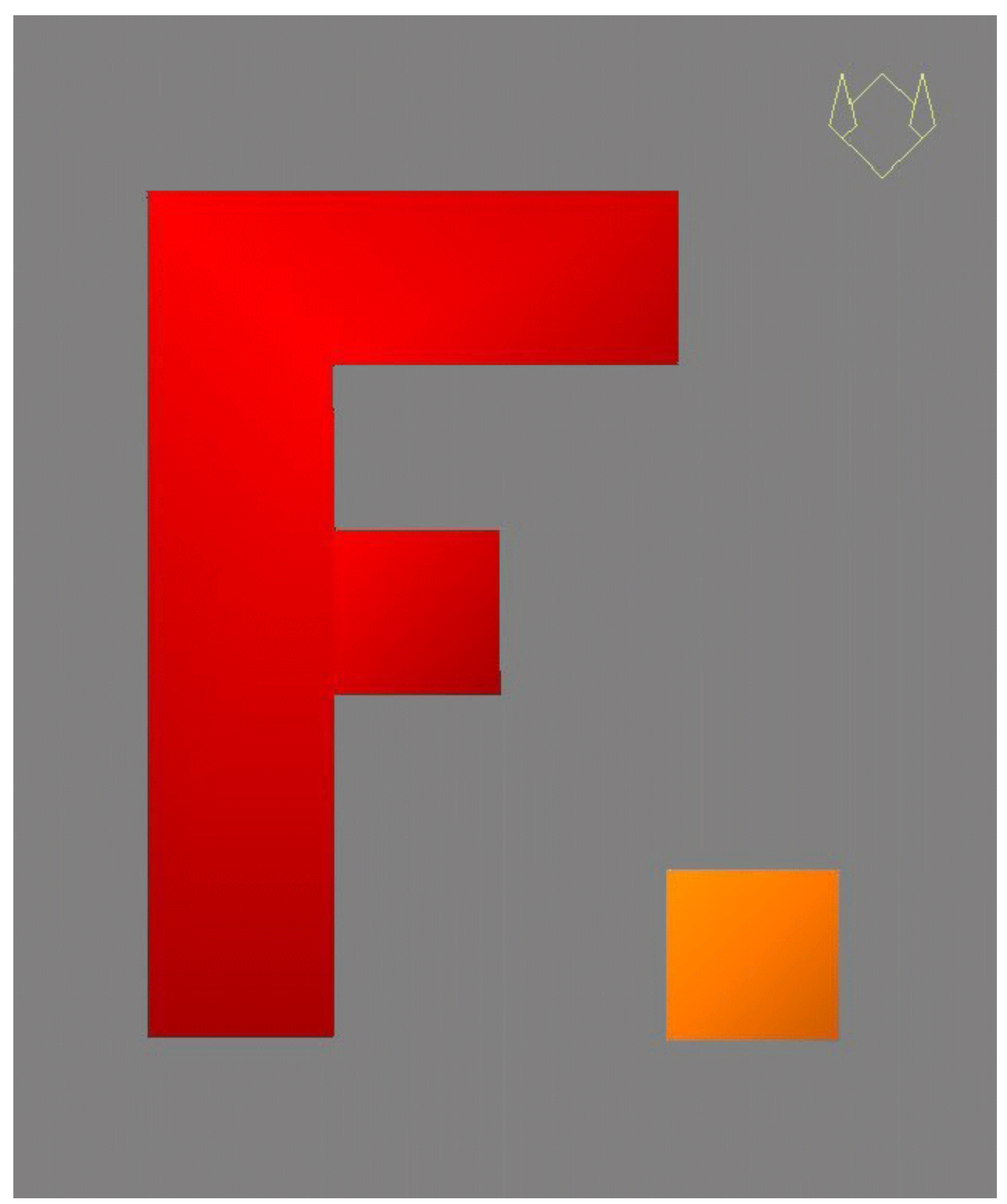

Fig. 2.2 Axial slice showing the ULF phantom in head-first supine position. The displayed F confirms the correct position representation. 

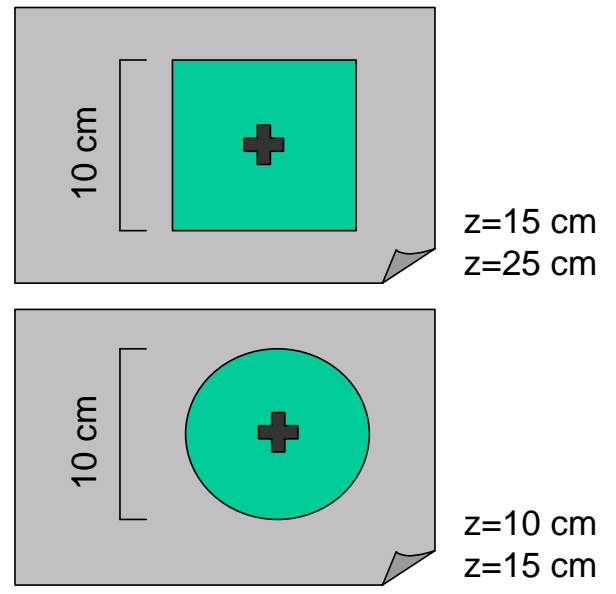

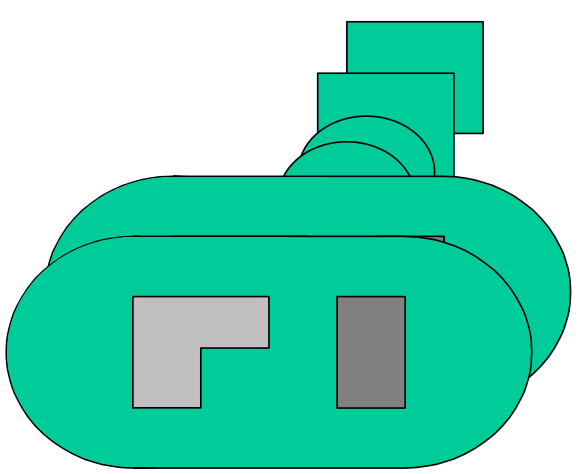

Semi-perspective view

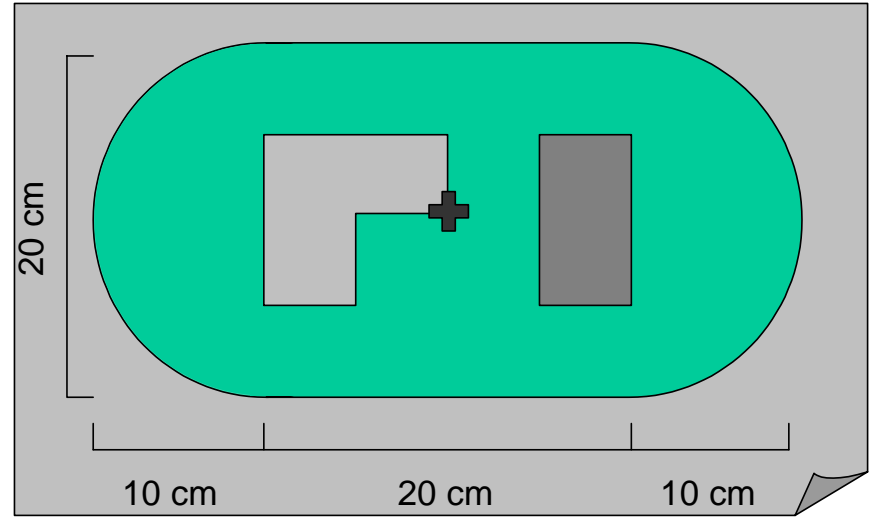

$\mathrm{z}=-10 \mathrm{~cm}$

$\mathrm{z}=0 \mathrm{~cm}$

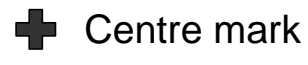

Fig. 2.3 Phantom to test the contour input. At the left side three contours are shown, each to be entered at two indicated axial levels. At the right side a pseudo-perspective view of the complete phantom is shown. 


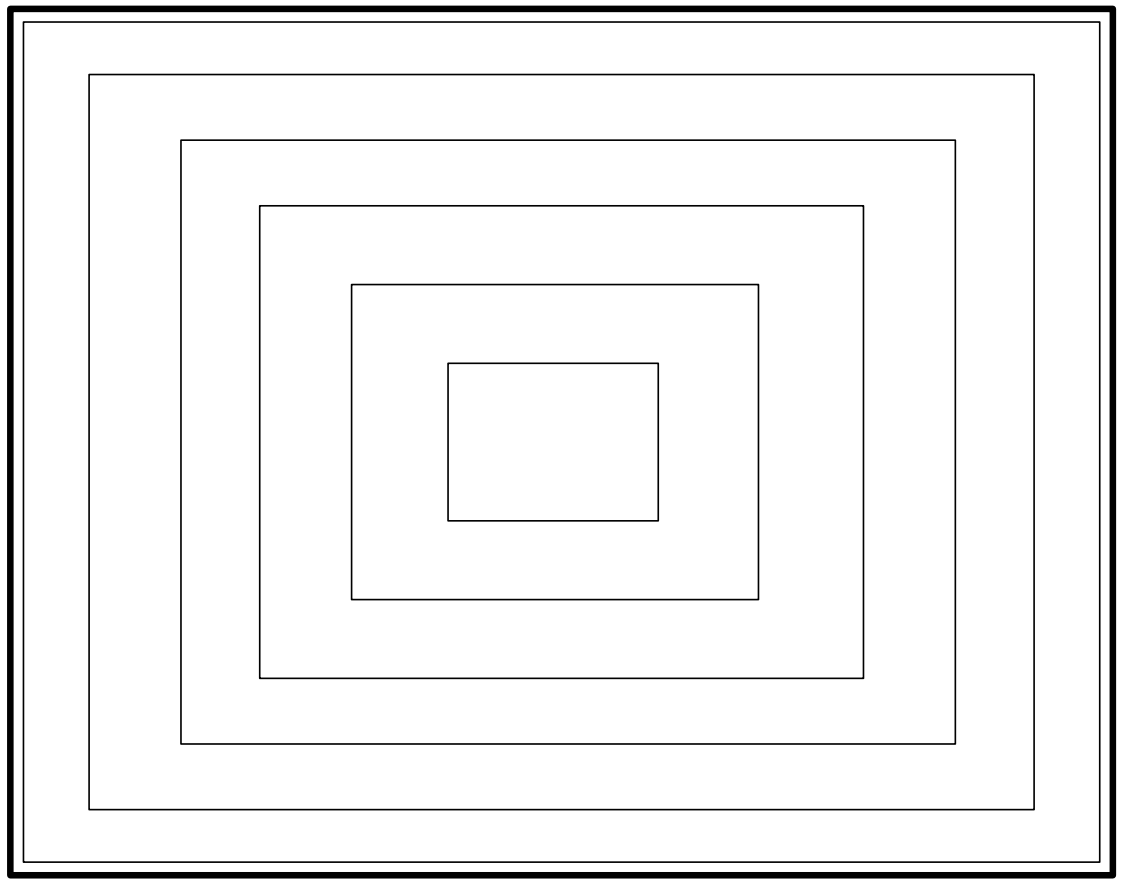

Digitizer active area

Fig. 2.4 Test set with six concentric rectangular contours to check the input of the entire active area of a digitizer. 


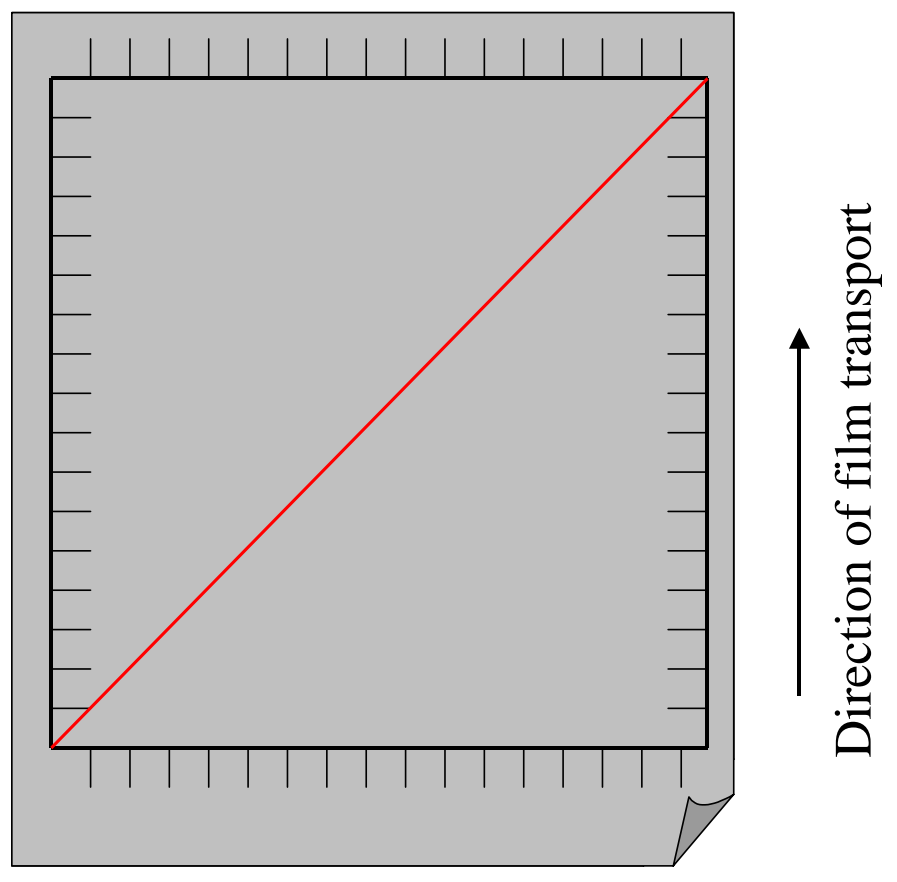

Fig. 2.5 Test set to check the linearity of a film scanner. Non-linear behaviour in either the transport direction or the direction perpendicular to that is noticed by a deformation of the straight diagonal line. The scales on the sides of this set allow quantification of the non-linearity. 

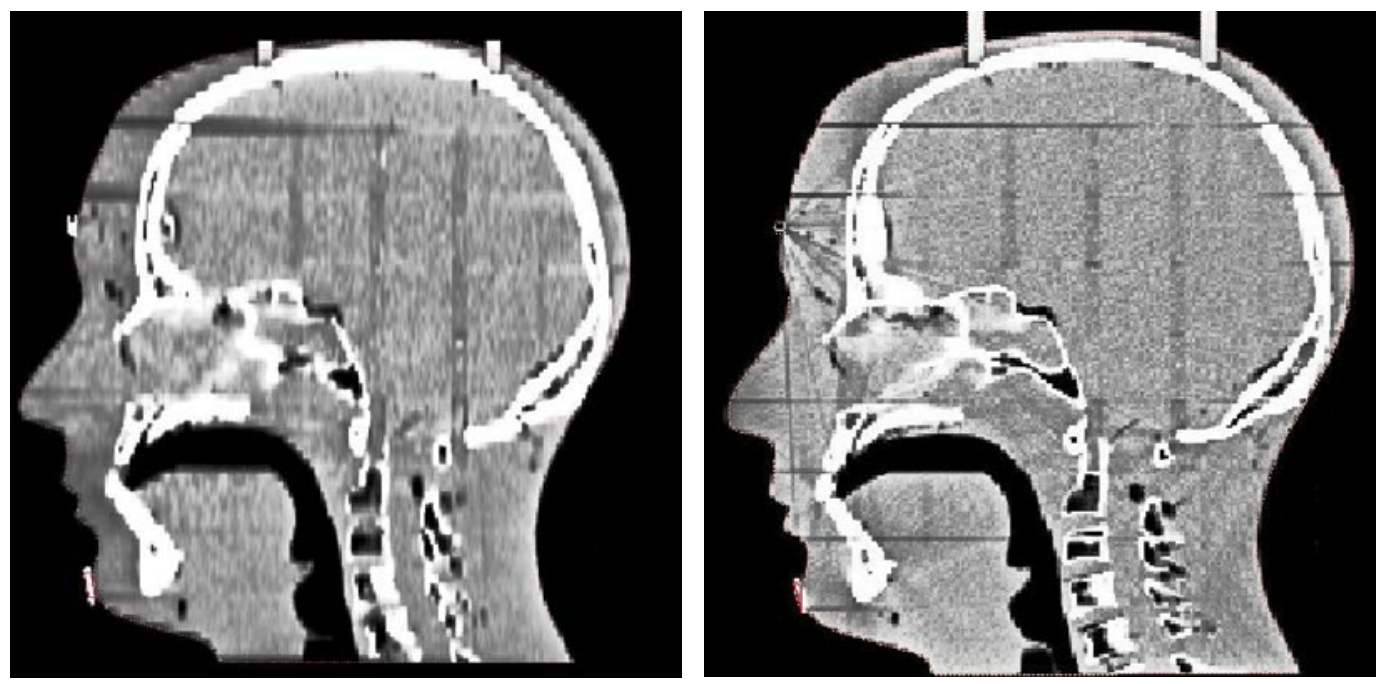

Fig. 2.6a (left) Sagittal reconstruction of the head of a Rando phantom . Fig. 2.6b (right) Slice from a scan of the Rando phantom in a sagittal orientation. 


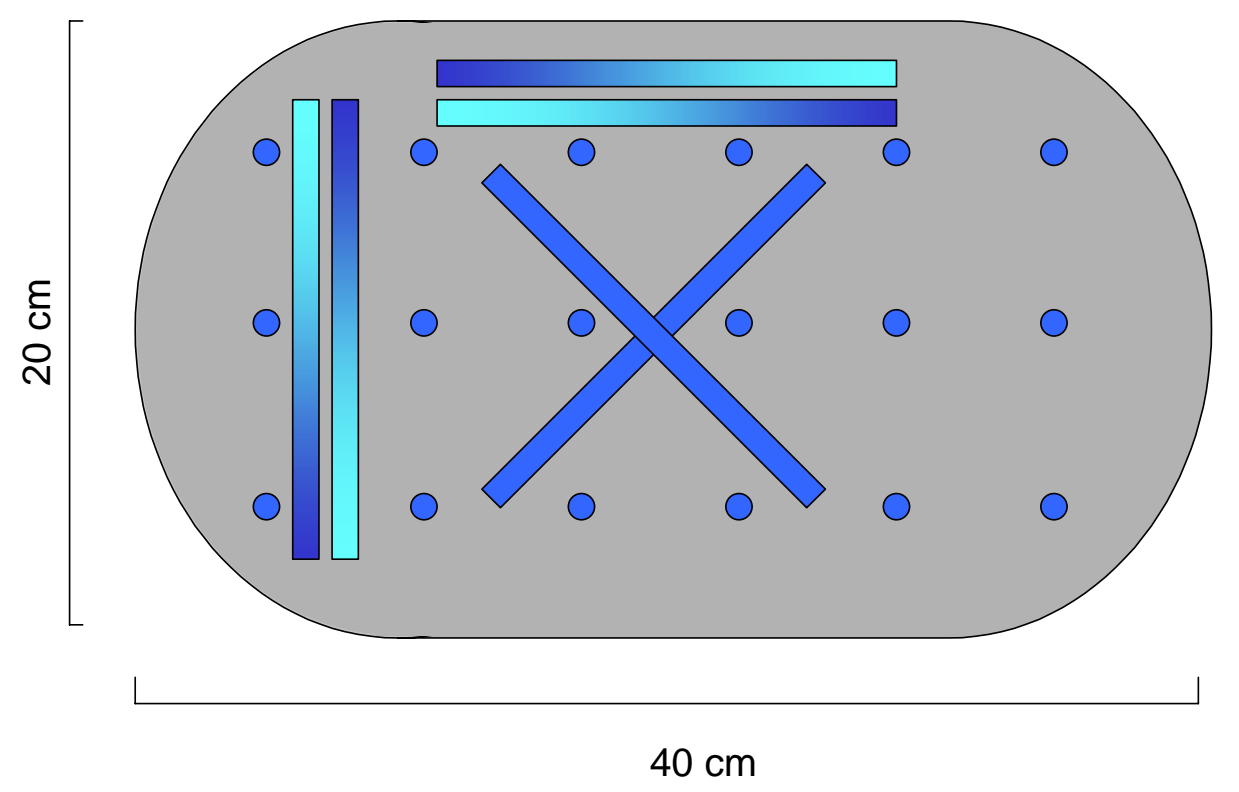

Fig. 2.7 Contour of a MRI test phantom. 18 rods run cranio-caudal, i.e. perpendicular to the plane of paper. The other six rods form an X when seen from the three main orientations, respectively. 


\section{Beam description}

External beam treatment planning requires the definition of geometrical properties of the specific treatment machines and beams in the configuration part of the TPS. During the treatment planning process beam arrangements are set up. Beam entries and directions are selected and field sizes and shapes are defined. In order to facilitate the beam set-up the user applies various types of beam and patient image display modes, e.g. images in axial or non-axial planes, beam's eye view (BEV) images and 3-D views. During beam set-up, the selected parameter values must correspond with the actual beam orientation in relation to the patient anatomy. In principle one can distinguish three aspects of beam description in a TPS: beam definition, beam display and beam geometry, which should be verified regarding functionality and accuracy. In practice, these aspects cannot be separated completely and some overlap is unavoidable.

In this chapter tests are presented to ensure that a treatment machine is defined correctly in the TPS, that beams are displayed correctly and that beam set-up options function correctly. The verification of dosimetric properties of treatment beams, which are also defined in the configuration part of the TPS, is discussed in chapter 4. For a description of 3D treatment planning systems see also [21].

\subsection{Beam definition}

In treatment machine configuration the beam parameters for a specific treatment machine must be defined, as well as their mechanical limitations. Some TPSs use a parameter convention system during interactive treatment planning, which is independent of the type of treatment machine (e.g. the convention system as defined in IEC Report 1217 [22]). Then for output on plot, print, etc. a conversion is provided to the beam parameters of the specific machine. Other TPSs allow direct definition of specific beam parameter systems for each treatment machine individually. Limits in beam parameter values are defined for a specific machine in order to simulate its mechanical movements and limitations and to avoid generation of impracticable treatment plans. These limits may be entered in the TPS either by using the general beam parameter system or the machine specific beam system.

Regarding electron beam collimation, some TPSs require the exact geometric data of the electron beam applicators, maybe including fixed air gap values between the applicator and the patient's skin. 


\subsubsection{Beam orientation}

\section{Scope}

To ensure that a particular treatment machine has been defined correctly regarding the geometrical parameters that define the orientation of the beam relative to the patient.

\section{Background}

The position and direction of a beam is defined by the location of the isocentre in the patient together with the gantry and table angles. The collimator angle defines the orientation of the beam around its direction. The collimator field size settings together with the source-axisdistance (SAD) and source-surface-distance (SSD) determine the outer beam size and its divergence. For electron beams it may be fixed applicator size and applicator-surface-distance that determine the latter two characteristics. Electron beam data may be stored for a set of discrete SSD values.

Correct handling by the TPS of the various beam orientation aspects depends on the entered parameter values and the correspondence of direction of translation or rotation to increase of values.

\section{Suggested tests}

a. Isocentre position

Position a beam with a rectangular shape on a 3-D-phantom with its isocentre $10 \mathrm{~cm}$ below the surface, but not at the origin of the phantom (fig 3.1). Select a gantry angle of $0^{\circ}$ (perpendicular incidence), a collimator angle of $0^{\circ}$ (standard position) and a table angle of $0^{\circ}$ (standard position). Verify the position of the isocentre position relative to the origin of the phantom. Check the SSD value as stated by the TPS.

b. Source-axis distance and field size

Measure the field size at the isocentre level in axial and sagittal planes and verify the agreement with the $X$ - and $Y$-values stated by the TPS.

Measure the field size at the phantom surface and verify the correct divergence. Repeat this test for various field sizes and check that the divergence varies linearly with field size.

c. Gantry rotation

Change the gantry angle from $0^{\circ}$ to $30^{\circ}$ and verify the correct direction of rotation. Verify the agreement of the beam angle on the plot with the value stated by the TPS.

d. Collimator rotation

Set the gantry angle to $0^{\circ}$ again and change the collimator angle to $30^{\circ}$. Verify the collimator 
angle and the correct direction of rotation by means of distance measurements in the beam display of the axial and the sagittal plane and in BEV display (fig. 3.2).

e. Table rotation

Set the collimator angle to $0^{\circ}$ again and change the table angle to $30^{\circ}$. Because the result of table rotation and collimator rotation are the same in BEV display for gantry angle $0^{\circ}$, one can verify the table angle and its direction of rotation using the field display in the axial and sagittal plane and in the BEV display.

f. Limits of parameters

Vary the SSD, the gantry, collimator and table angle to minimum and maximum values and check the system's response. This is to verify that entered limit values are taken into account correctly.

g. Electron applicator collision

If the geometry of the applicator is defined in the TPS, the system may check for collision with the skin. Verify this for various combinations of SSD, gantry, collimator and table angles.

\subsubsection{Beam size and shape}

\section{Scope}

To ensure that a particular treatment machine has been defined correctly regarding the geometrical parameters of the beam size shaping devices.

\section{Background}

The outer size of a photon beam is defined by the four jaws $\left(X_{1}, X_{2}, Y_{1}, Y_{2}\right)$ and the shape by the leaves of the multi-leave-collimator (MLC) or by a shielding block. The outer size of an electron beam may also be defined by the jaws or by a fixed size applicator; the shape is determined by a shielding insert.

\section{Suggested tests}

a. Field size of photon beams

Continue with the beam and phantom set-up as defined in test 3.1.1 a. Change the settings of the four separate jaws and verify the correspondence with $X_{1}, X_{2}, Y_{1}$ and $Y_{2}$. Select a range of settings of the four parameters, measure the distances at the definition level (in most cases isocentre level) in the display of the axial and sagittal plane and in the BEV display (fig. 3.3.) Compare to measured values to the parameter values stated by the TPS.

b. Field size of electron beams 
If the field sizes are defined by the jaws, repeat test a. for the electron beams. If the field sizes are defined by fixed applicator sizes, verify that the field sizes correspond to the selected applicator at definition level. This may either be the level of the isocentre or at the level of the bottom end of the applicator.

c. Multi-leaf collimator settings

Define an irregular field shape by the MLC, using an automated shaping option. Check the number of leaves used; the position, numbering and direction ( $X$ or $Y$ ) of the leaves; the leaf width, over-travel and maximum leaf position and interleaf distance. Check whether the jaws are automatically fitted to the largest leaf positions in the field.

d. Block position and size.

Define a field shape by a block, using a manual method specifying BEV field co-ordinates. Measure the block size at the isocentre level in axial and sagittal planes and verify the agreement with the entered shape. Measure the block size at the phantom surface and verify the correct divergence.

e. Limits of parameters

Vary the field size of the jaws in $X$ and $Y$ to minimum and maximum values, the over-travel of jaws for asymmetric fields to maximum value (for open and wedged fields), the MLC leaves settings to minimum and maximum positions, and check the system's response. Check the allowed applicator sizes for electron beams if applicable. This is to verify that entered limit values are taken into account correctly

f. Validity check

When a field shape has been conformed to a target volume, either by MLC or block, this is related to a specific beam orientation. Any change in gantry, collimator or table angle, should be followed by a warning by the TPS.

\subsubsection{Beam modifiers}

\section{Scope}

To ensure that a particular treatment machine has been defined correctly regarding the geometrical parameters of the beam modifiers.

\section{Background}

The fluence of a photon beam may be modified by a solid wedge that is part of the treatment head of the linear accelerator or that is inserted in a tray holder attached to the treatment head. The orientation of the wedge is determined by the collimator rotation of the beam and if possible 
by the insertion direction. Photon beam fluence may also be modified by a compensator inserted in a tray holder. Furthermore, in both photon and electron beams bolus material may be placed on the patient's skin to modify the fluence.

In the TPS bolus may be treated as part of the beam or as part of the patient. In this chapter we consider bolus as a beam related modifier; it is a layer with a fixed height and a specific density, initially defined within the aperture of the beam.

\section{Suggested tests}

a. Wedge insertion:

Check the possible directions of insertion of a wedge in a photon beam. Verify that the wedge direction changes with the collimator rotation angle, but that the insertion direction remains the same.

b. Bolus definition.

Define a bolus in a beam using BEV field co-ordinates. Verify the bolus presentation at the surface of the phantom in axial and sagittal planes; the generated bolus should exactly cover the defined area within the beam. Check bolus density and height throughout the entire beam aperture using CT-number and distance measurements.

c. Validity check

When a bolus has been defined in a beam, its position is related to a specific beam orientation. Any change in gantry, collimator or table angle, should be followed by a warning by the TPS.

\subsubsection{Beam parameter conversion}

\section{Scope}

To ensure that the conversion of beam parameters from the convention system of the TPS to the convention system of a particular treatment machine is carried out correctly. The test also verifies correct beam parameter transport to the machine and actual beam set-up. Besides this test may be used to check the BEV field set-up for beams with non-zero table angle. A detailed description on co-ordinate transformations for an accelerator is given in [23].

\section{Background}

If the TPS uses a fixed beam parameter system for the treatment planning part, one should check the conversion for the specific treatment machine. Non-consistent TPS output due to incorrect beam definition might lead to errors in preparing the actual treatment of a patient. For 
example, the radiation technologists at the treatment machine should not need to guess whether a table angle is $+5^{\circ}$ or $-5^{\circ}$, or have to make the conversion of the TPS output themselves. Especially when treatment parameters are sent directly to the treatment machine and the patient irradiation will be performed using automatic set-up, careful validation is essential.

This test also offers the possibility to verify the BEV display for particular fields with non-zero table angle. Therefore we define a non-axial beam orientation, using rotated gantry, collimator and table and an asymmetric field of which the field shape is fitted to a tilted 3D-target volume in a phantom.

\section{Suggested tests}

a. Test phantom

Define a rectangular phantom in 3-D and a target volume that extends in the IEC y-direction with an angle of $30^{\circ}$ to the table surface (intersection with table surface in-y-direction). The target volume has a cylindrical shape with a small cross section, which models for instance an oesophagus tumour.

b. Test beam

Define a beam with non-zero table angle, with its isocentre at the centre of the target volume and the beam axis in a plane perpendicular to the target volume. Rotate the collimator to let the symmetry axis of the field coincide with the projection of the target.

Select a gantry angle of $41^{\circ}$, a collimator angle of $49^{\circ}$ and a table angle of $41^{\circ}$ (IEC-scales).

c. TPS beam orientation

Verify in the TPS that the symmetry axis of the field coincides with the longitudinal axis of the target volume. Transfer this beam set-up to the actual treatment machine.

d. Test experiment

Position a piece of paper, with the drawing of the target area at $30^{\circ}$ to one of its sides, perpendicular on the table surface. Set up the beam, manually or automatically, with the output parameter values of the TPS.

e. Actual beam orientation

Verify that the symmetry axis of the field coincides with the longitudinal axis of the target volume (fig. 3.4).

Any error in the configuration of the beam in the TPS regarding gantry, collimator, table angle or jaw settings will be detected by this test. This test may be extended with a MLC defined field shape setting and a wedge insertion. 


\subsection{Beam display}

\section{Scope}

To ensure that the geometry of a treatment beam is displayed in correspondence with its parameter values and correctly in relation to the anatomy of the patient.

Background:

Beam display in a TPS provides the user with views on the orientation and shape of the treatment beams in relation to the patient. These display options are used during beam set-up in the TPS, taking the position and shape of the target volume and of organs at risk into account. Beams are displayed in axial, sagittal, coronal and oblique planes, in BEV, in digitally reconstructed radiographs (DRRs) and in 3-D views. The beam display may include the beam's central axis, the diverging beam edges, the diverging aperture edges defined by a MLC or block and wedges. Display options may also be used for beam matching in the TPS and for support or verification of actual beam set-up on a simulator or treatment machine.

\section{Suggested tests}

a. Test phantom

Define a water phantom of adequate size; e.g. width $40 \mathrm{~cm}$, height $25 \mathrm{~cm}$ and length $30 \mathrm{~cm}$, symmetrically around the origin. Add two cylinders with different densities (e.g.: lung density of $0.3 \mathrm{~g} / \mathrm{cm}^{3}$ and bone density of $1.8 \mathrm{~g} / \mathrm{cm}^{3}$ ).

b. BEV display

Position a beam with a symmetric field $(e .9 .10 \mathrm{~cm} \times 10 \mathrm{~cm})$ with its isocentre in the phantom's origin. Select a gantry, collimator and table angle of $0^{\circ}$. Verify in the BEV display the correct projection of the phantom's contours and the size and position of the field. Repeat this test for a set of SSD values.

c. Various displays

Use the same beam but change to an asymmetric field and to a gantry angle of $30^{\circ}$. Keep the collimator and table angle equal to $0^{\circ}$ and insert a wedge (fig 3.5).

e. Verify the correct display of the beam's axis, divergence lines, field aperture and wedge direction relative to the phantom contours in an axial and coronal plane through the isocentre, in BEV and in 3-D view.

f. DRR display

Verify the agreement of beam position between the DRR and the BEV. Verify the SSD as stated by the TPS

g. Block graphics 
Define a block shape manually using BEV field coordinates. Verify the block presentation in the various display types.

h. MLC graphics

Define a MLC field shape manually using BEV coordinates. Verify the MLC presentation in the various display types.

Repeat tests a to $\mathrm{h}$ for various gantry, collimator and table angles. In particular check whether the 'AP/PA', 'cranial/caudal' and 'left/right' indicators are correct in these displays.

i. Bolus

Define a bolus manually. Verify the bolus display on the patient's surface in BEV, axial, coronal and sagittal planes and in 3D view.

j. Light field projection

Construct a geometrical phantom with a $2 \mathrm{~cm}$ slice distance and a top surface at an angle of $45^{\circ}$ with a horizontal plane. Define a beam with $20 \mathrm{~cm} \times 20 \mathrm{~cm}$ field size and gantry angle $0^{0}$ incident on this angled surface. Compare the dimensions of the observed light field shape with calculations or with a set-up on a simulator (fig 3.6).

\subsection{Beam geometry}

Scope

To ensure correct orientation, size and shape of a beam in relation to the patient after using various beam set-up functions of the TPS.

\section{Background}

During the treatment planning process the user strongly relies on the geometrical accuracy of the beam set-up functions of the TPS. In sections 3.1 and 3.2 tests on beam parameters have been suggested to verify aspects primarily related to correct beam definition and display functions. In this section we focus on aspects related to correct beam manipulation functions and some issues not addressed previously. The tests should verify consistency in the beam parameters between various set-up or display modes and between the various input modes of parameter values. In addition attention is paid to tests of DRRs of which the most important features during beam set-up are linearity and divergence. DRR tests are also reported in [24, 
25].

\section{Suggested tests}

a. Phantom and beam

Continue with the phantom and beam set-up as defined in 3.1.1.a. Define a 3-D rectangular target volume.

b. Beam move

Move the beam by mouse in axial plane or BEV display mode. Check the accuracy of the displacement using coordinate and distance measurements. Repeat this test entering values by keyboard .

c. Beam functions

Use the various beam input, change and edit functions like shift beam, mirror beam, oppose beam, change z-coordinate, etc. Check the accuracy of the results in relation to the patient co-ordinate system and the consistency between results using different functions.

d. Change SSD.

Vary the SSD of a fixed SSD beam (if an option in the TPS) and check the field size at the surface and the divergence. Verify for SSD values smaller than the SAD the agreement in display of the fixed SSD beam compared to an isocentric set-up beam.

e. Automated block shape

Define a block in BEV, conformal around the target, using a predefined margin. Verify the position of the block, in an axial and coronal plane.

f. Automated MLC shape.

Define a MLC shaped field in BEV, conformal around the target, using a predefined margin. Verify the position of the leaves of the MLC in an axial and coronal plane. Repeat this for inside, middle and outside settings of the leaves. Verify the algorithm that sets the jaws positions close to the most outer leaves.

g. Block functions

Use the various block input, change and edit-functions such as: shift block, mirror block, copy block, read block from file, from film, etc. Check the accuracy of the results in relation to the patient co-ordinate system and the consistency between results using different functions.

h. DRR generation.

Create a phantom for DRR generation checks. This phantom has different densities shaped by the size and the divergence of a pre-defined beam (see fig 3.7.). The black volumes are empty (density 0), the grey volumes have density 1 (water) and the white have density 2. Position the beam exactly at the centre of the diverging volumes (see fig. 3.7.), calculate a 
DRR and verify the calculated grey value and the divergence by the sharp projected edges of the volumes. The result should be as shown in fig 3.8.: the same grey-level for the regions 3,4 and 5 and sharp transitions between the other regions. Repeat this test for other SSDand field size settings and using the same and different phantoms .

i. Bolus generation

Bolus may be defined slice by slice or in the BEV. BEV definition is much easier than the slice by slice procedure. Anyhow, the transfer from BEV definition to positions in axial slices regarding position, size (extension) and the thickness of the bolus should be verified.

Define a bolus in BEV mode and verify the correct position and height in axial planes. Verify the connection of the bolus to the body contour (no air gaps in between) and the extension of the bolus in the length direction of the patient (number of slices containing the bolus) 


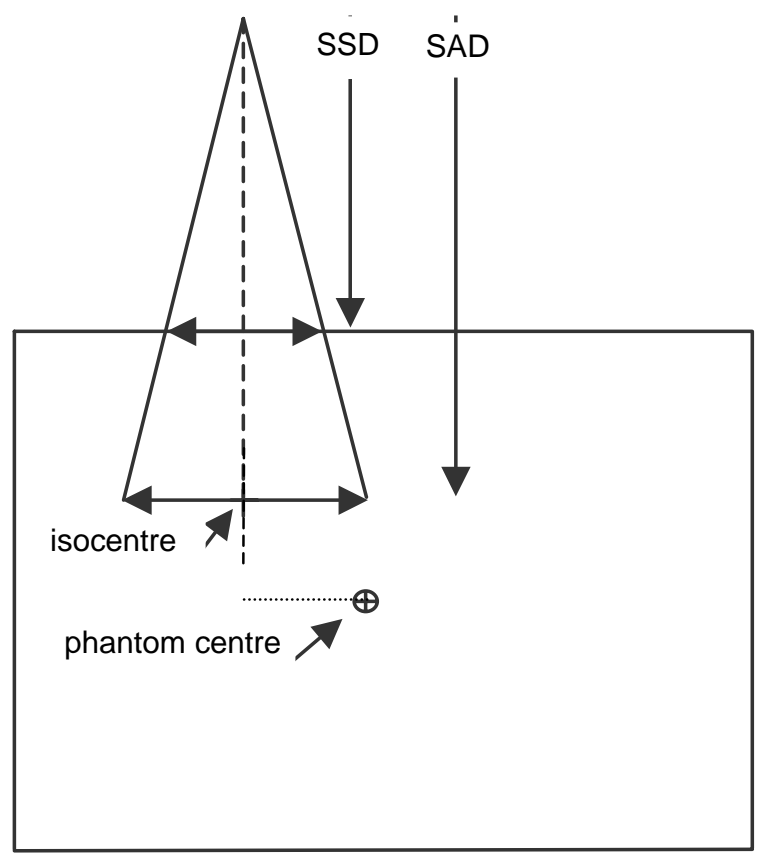

Fig 3.1.Isocentre co-ordinate and field geometry test 


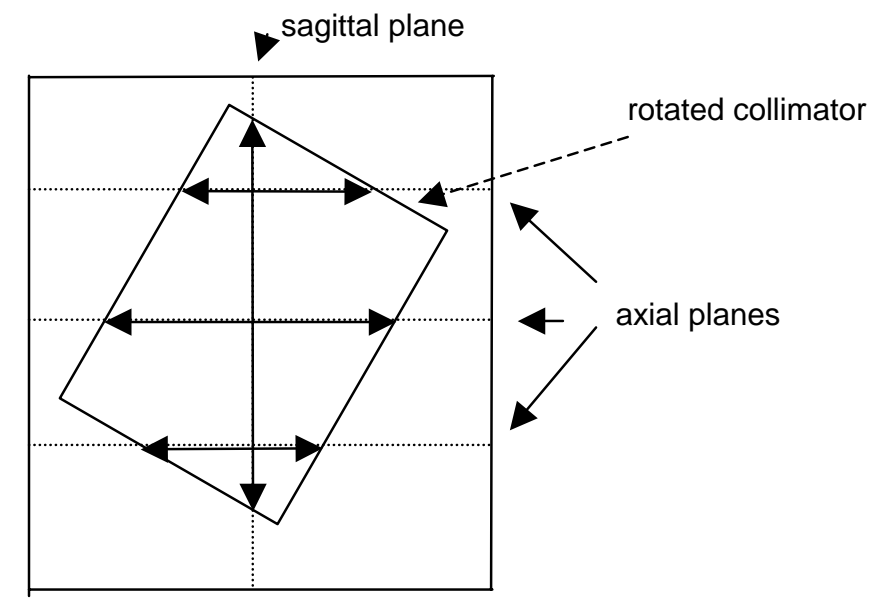

Fig 3.2. BEV field display. Agreement between field shape in BEV and axial plane. 


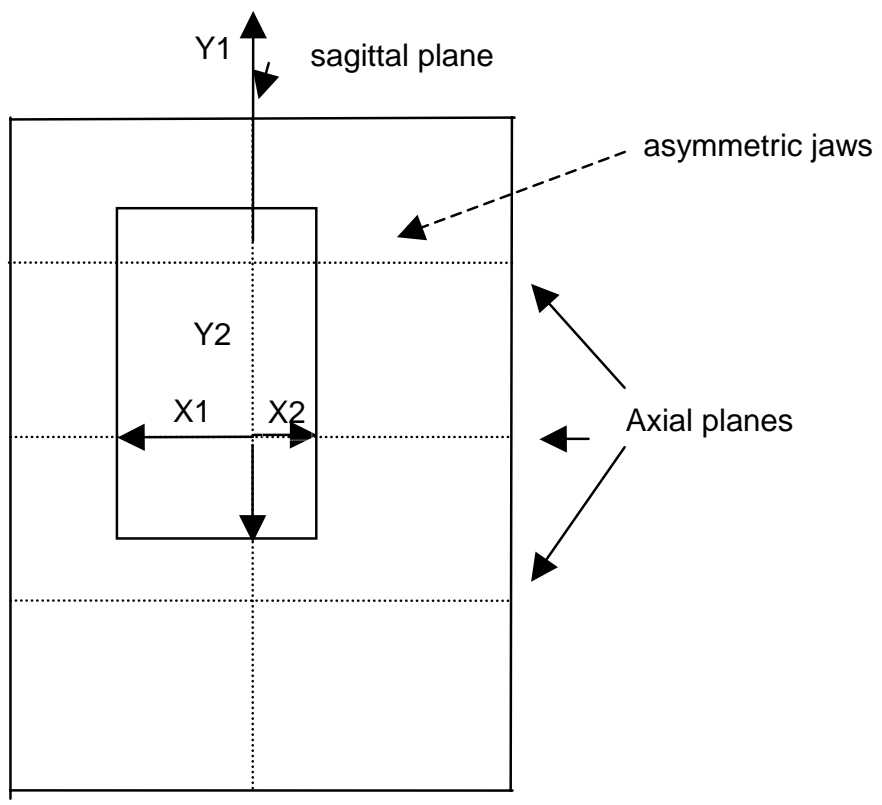

Fig 3.3. Jaw setting and beam co-ordinate test 


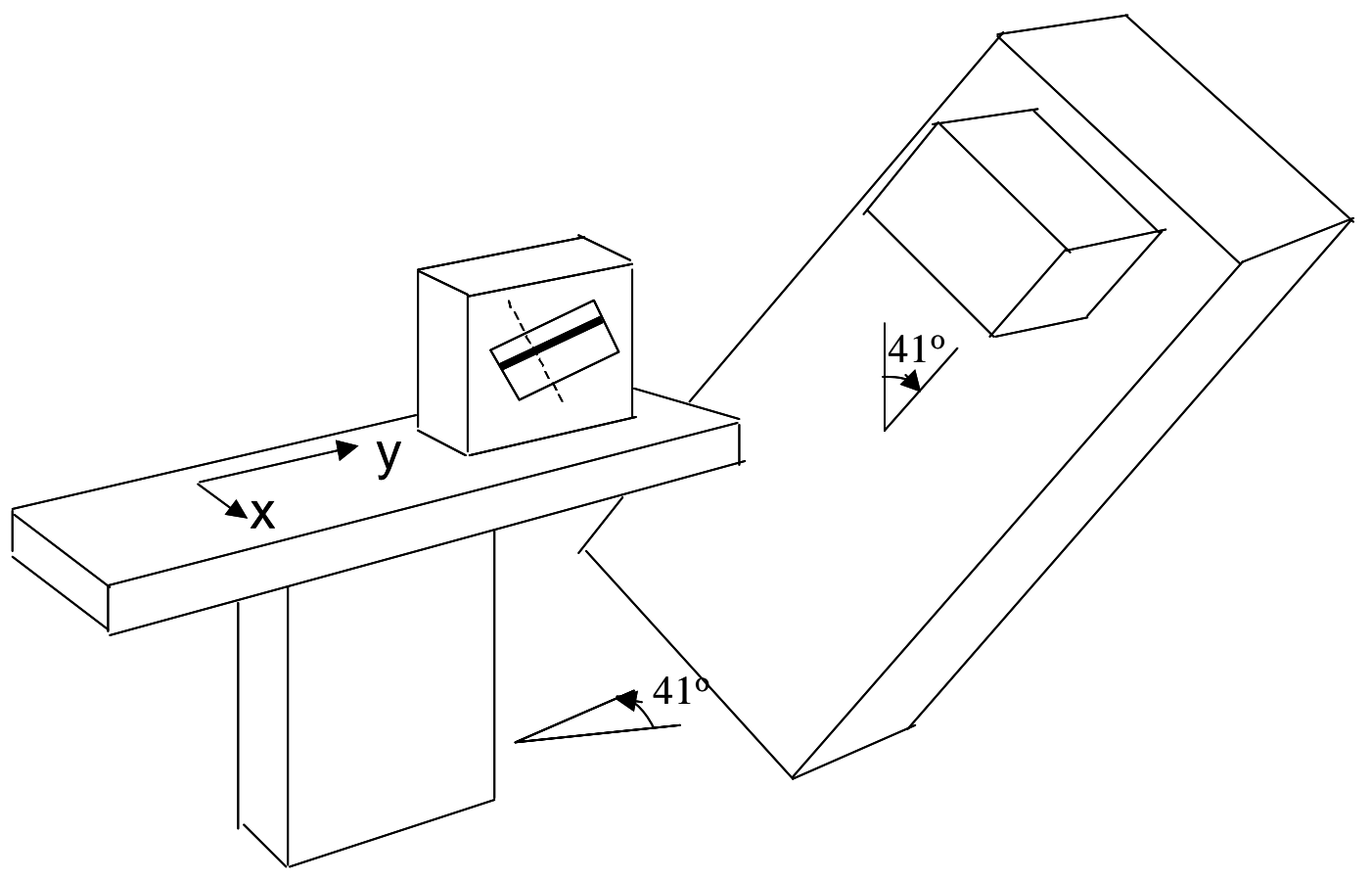

Fig 3.4. Conversion of TPS co-ordinates to accelerator co-ordinates 


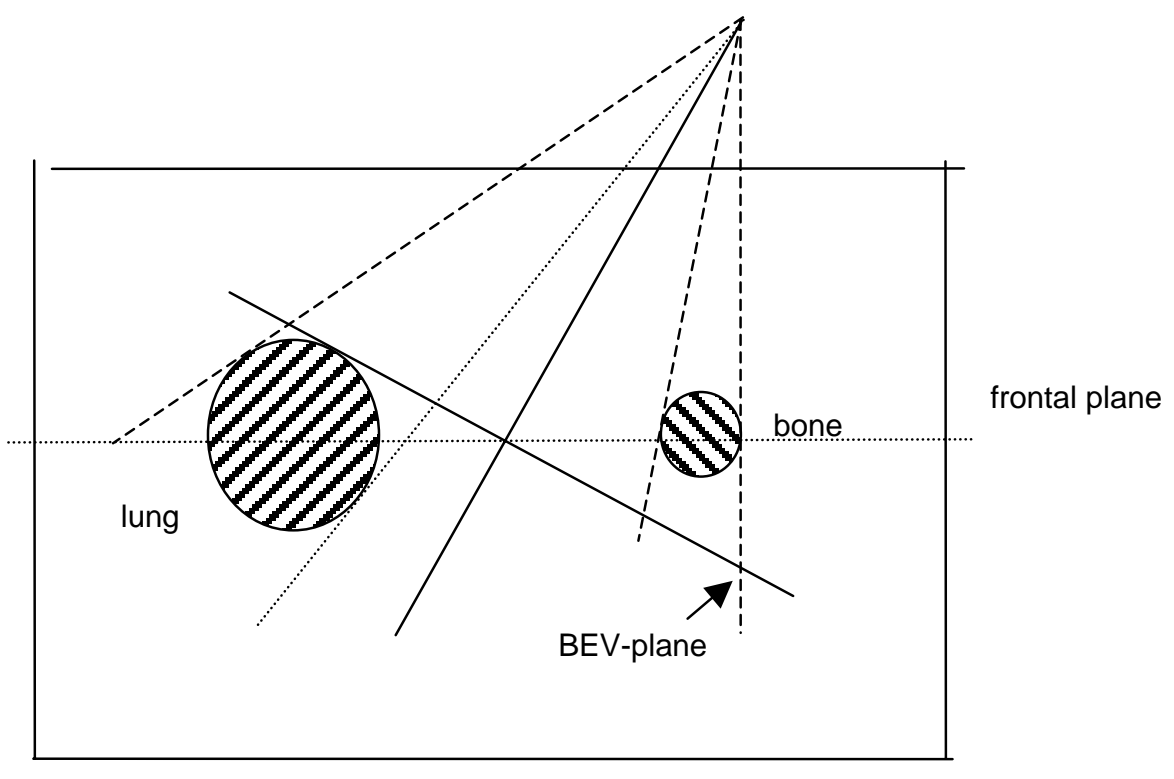

Fig 3.5.: Beam display test 


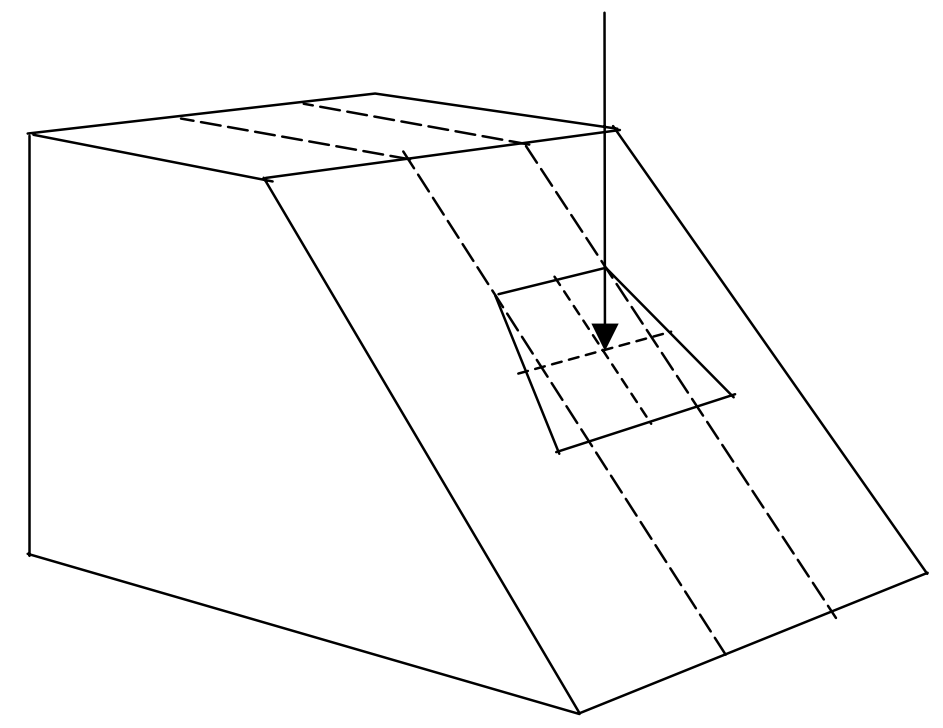

Fig 3.6.: Light field shape test 


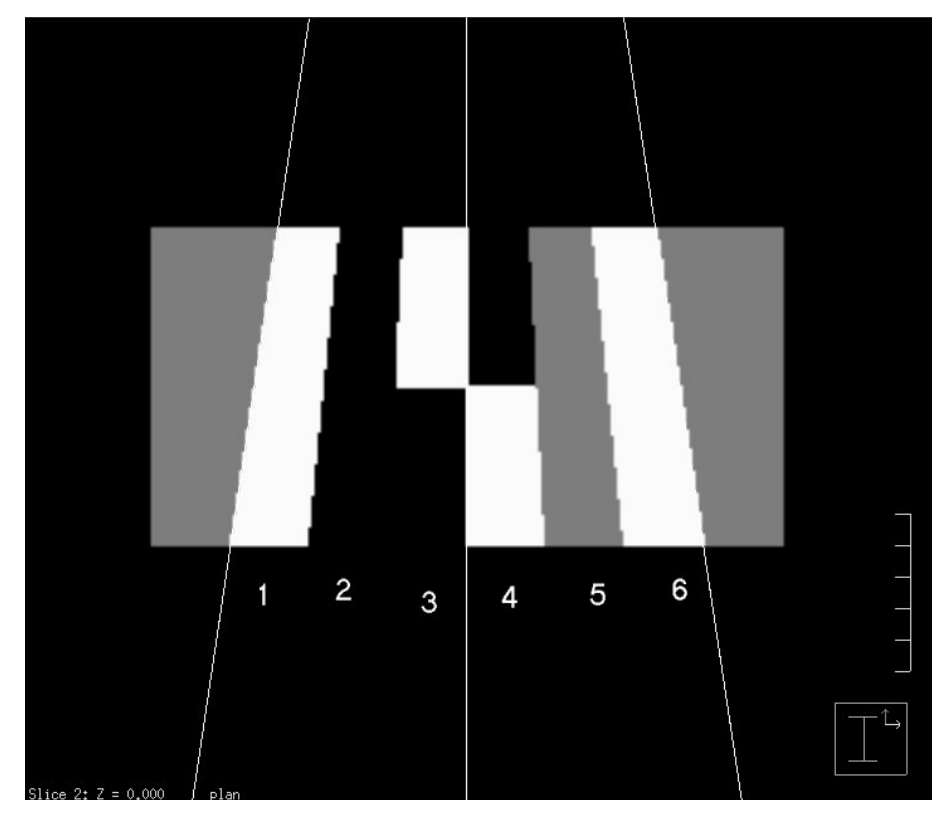

Fig 3.7. Phantom for DRR-test 


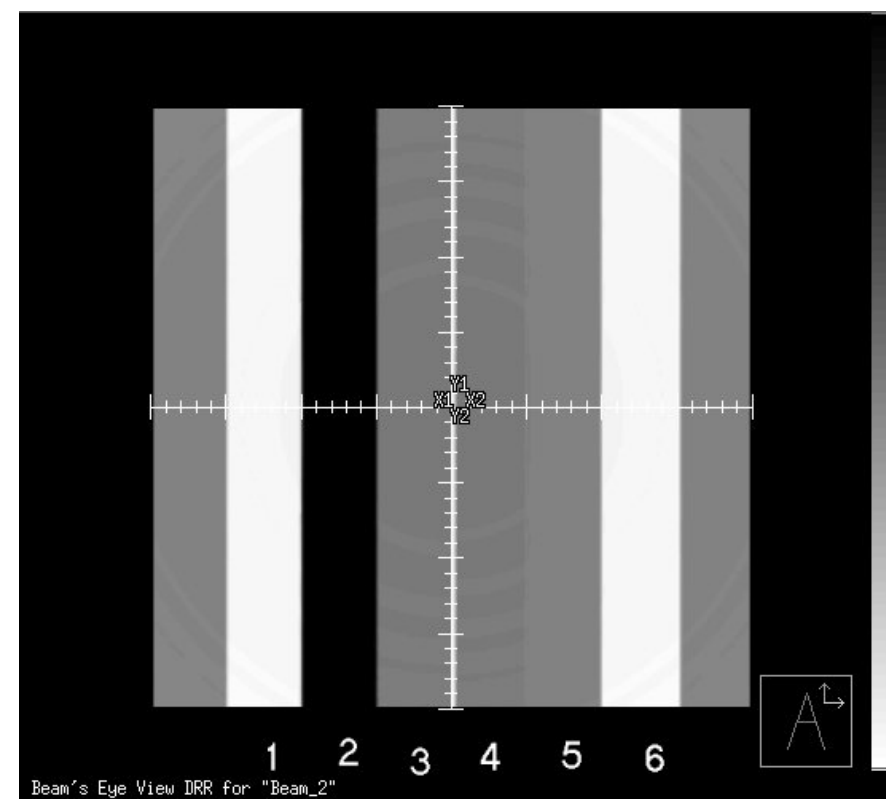

Fig 3.8. Result of DRR-test 


\section{Dose calculation}

QA of a TPS requires methods to check and verify the accuracy of the dose calculations for photon and electron beams performed by a specific release of that system. The accuracy of dose firstly affects the uncertainty in the evaluation of treatment plans, for which target dose homogeneity and dose to organs at risk are the criteria. This concerns the accuracy in the calculation of relative dose distributions. Secondly the accuracy of dose affects the uncertainty in dose delivery, in particular the dose to the treatment plan normalization point. This concerns the accuracy in the calculation of monitor units (MUs) for a specified absolute dose value. The uncertainty in dose delivery to an arbitrary point concerns the combined accuracy in the calculation of MUs and relative dose distributions.

It should be noted that any dose verification measurement includes uncertainties due to the measurement itself as a result of limitations of the detector system, the set-up used for the measurements and the output of the treatment machine. Estimates of the measurement uncertainty should be included in any experimental verification of a dose calculation algorithm. In order to exclude the influence of output variation of the treatment machine on the result of the test, it is recommended to always perform an absolute dose measurement under reference conditions, e.g. at $10 \mathrm{~cm}$ depth in a $10 \mathrm{~cm} \times 10 \mathrm{~cm}$ field at $100 \mathrm{~cm}$ SDD, during a series of dose measurements. By also irradiating the detector used for the verification measurements, for instance a small ionization chamber or a diode, under these reference conditions, it is possible to convert the verification measurements to absolute dose values. In this way both relative dose distributions and absolute dose per $\mathrm{MU}$ at the treatment plan specification point are verified simultaneously during a specific test.

The relationship between dosimetric tests to be performed by the vendor, user groups and an individual user is in principle identical to that for other tests of that system. This has been elucidated in Chapter 1. Dosimetric accuracy depends on the quality of the beam data input (Section 4.1) and the dose calculation algorithms (Section 4.2). The set of verification measurements for photon beams presented in this report is based on a set of test data that was previously proposed by the American Association of Physicists in Medicine Task Group 23 (AAPM [4]). This set has been modified to include the new possibilities offered by more modern radiation therapy equipment (Venselaar and Welleweerd [26]). Approaches to handle differences between measurements and calculations will be discussed in Section 4.3. Finally criteria for acceptance are presented in Appendix A.4.1. 


\subsection{Beam data input}

\section{Scope}

To ensure the use of consistent datasets for beam modelling in a TPS and for the purpose of algorithm testing. These datasets are first defined, including the ways to handle the data in clinical routine.

\subsubsection{Basic beam data}

\section{Background}

It should be clearly indicated for which purpose a set of beam data is acquired. For example, basic beam data are entered in a TPS to model the beam. Other beam data can be used, for instance, for checking the outcome of dose calculations against directly measured data (Section 4.2). The latter data may consist of dose data, in Gy, for a specified number of MUs, determined at several points in a phantom for various clinically relevant beam geometries. We will refer to such data as test beam data. If the geometry of these beams is the same as the geometry of the beams of the basic beam dataset (e.g., open square fields), the data should be identical. For other geometries consistency should exist between the data of both sets.

Basic beam data are often collected over a prolonged period of time and by different investigators for a variety of, practical, reasons. Uncertainties in beam data caused by time intervals between measurements and by differences in procedures should be minimized as much as possible, in particular by using the same detector set. One should aim at a reproducibility of the data in: absolute dose values at the reference point to within 1.0\%; PDD values to within $0.5 \%$; dose profiles to within $1.0 \%$.

\section{Suggested tests}

a. Verify the consistency of the datasets by direct inspection of measured data, either in tabular or graphical form.

b. Check the (versions of) datasets for differences. If any differences are found, these should be analyzed and corrected.

c. The final result of these procedures is a complete reference set of beam describing data that can be used as input data for a TPS. 
In Appendix A.4.2 a more detailed description is given of the set of basic beam data. It should be noted that different types of TPSs might require different sets of beam data while also the format of the input data might differ.

\subsubsection{Documentation of beam data}

\section{Background}

The purpose of documentation is to have a transparent system describing in detail how the data have been obtained, how data have been implemented, and how these data have been stored. Other users should be able to reproduce the entire process of data manipulation.

\section{Suggested steps}

a. At data collection, prepare a complete written description of: dates and time (sequence) of measurements; names of the investigators; methods of measurement; equipment used for data collection; software version of this equipment; methods used for data manipulation, like averaging, smoothing, etc..

b. Store a copy of the original data before any data manipulation is started.

C. Document the final results on print and/or plot and store in a digital form, e.g., on a CD, if applicable.

d. Document where data are filed. Special attention should be given to electronic files, i.e. file names, back-up files, version numbers, although such transparancy is also required for paper files.

e. Document all events, measurements and changes concerning the TPS in a logbook.

\subsubsection{Verification of the input process}

\section{Background}

One should verify whether the data were implemented correctly in the TPS. Often the system can generate prints and/or plots of the input data, sometimes in combination with the result of beam modelling. The correspondence between calculated and input data should be checked immediately after the beam modelling process for a number of fields. These checks should encompass the beam profiles, percentage depth dose curves, output factors and wedge factors. The comparison is carried out for relatively simple geometries only, i.e. in the large water phantom and without inhomogeneities. 


\section{Suggested steps}

a. Check the calculations in simple geometries. Only if these comparisons are satisfactory, next steps in beam modelling can be made.

b. Perform the same comparison in cases where datasets are copied, for example after installation of a new software release.

c. If the set of input data and the results of beam modelling by the TPS are accepted, this set should be considered as the basic beam dataset and uniquely documented. This set should be used for future quality control of the TPS, but also of the treatment machine.

Many TPSs offer special tools to review the data, for example on the display monitor. Graphs of the data versus field size and/or depth of measuerment are generally shown. Irregularities can easily be found with these tools. These irregularities should be eliminated before accepting a beam model for patient treatment planning. Such irregularities may require a renewed process of measuring or collecting (a part of) the dataset. If the manufacturer takes care of the beam modelling process, others may perform several (parts of these) steps, but the final result should always be checked and approved by the user.

\subsection{Dose calculations}

\section{Scope}

To determine the accuracy of the dose calculation performed by the TPS. One may distinguish verification of the type of dose calculation algorithm, and its specific implementation in the TPS. In addition, one should establish the limits of clinical use; i.e. the range of application with acceptable accuracy.

\subsubsection{General requirements}

\section{Background}

The type of work described in this section 4.2 can be shared by an individual user of a TPS and by user groups of the same TPS or joint working parties. For example, a group of users may define a set of basic beam data of a commonly used type of treatment machine. Then, a set of tests may be developed of which the results are applicable for all users of this system and can be shared and communicated. In this case, the tests for a particular radiotherapy 
department may be limited to a subset of the recommended set of tests. Otherwise the department itself should undertake complete testing.

\section{Suggestions}

a. The user should verify that all dose calculation algorithms and specific implementation aspects included in the TPS are well documented. The vendor should provide a description of all factors taken into account in the algorithms, the mathematical equations which form the basis of the calculation, and the limits of all variables used in the equations. The description should include references to the relevant literature.

b. Where a choice of algorithms is provided for a particular calculation, the instructions for use shall clearly identify the relative advantages and disadvantages of the different algorithms.

c. The uncertainty associated with the measured data, data entry and output should be understood by the user, through publications and/or vendor documentation.

The comparisons between measurement and calculation should be performed and analyzed applying welldefined criteria. A recommended set of criteria is discussed in Appendix A.4.1.

\subsubsection{User group tests}

\section{Background}

In a 3-D, as well as in a 2-D TPS, dose distributions are calculated for far more complex situations than those in which the basic beam data were measured. Therefore, additional measurements are needed to verify the accuracy of the algorithms for cases where interpolations and extrapolations are applied, covering the range of clinical applications in the radiotherapy department: the test beam data set. Such a set of measured data should be modular and 'dynamic', i.e. new tests can be added to the set, if new technical developments either in treatment machine design or in TPS software become available. The data should be generated in close co-operation with the vendor of that system and made available, in principle, to all users of that type of TPS.

\section{Suggested tests}

a. Describe the measurements needed for a complete test of the modeled beams which are applied clinically. For pragmatic reasons these measurements can best be performed during the commissioning of an accelerator. 
b. The beam data should consist of absolute dose values at specific points, central axis depth dose values and beam profiles for:

- square and elongated fields;

- different SSDs;

- extremely large and extremely small field sizes;

- beam modifiers: wedges, trays, trays with blocks, inserts for electron beams;

- oblique incidence, tangential beams;

- inhomogeneities: bone, air cavities or lung;

- asymmetrical collimator settings (with and without wedges);

- MLC shaped fields;

- collimator rotation.

c. Test configurations are proposed in detail for photon and electron beams in Appendix A.4.2. These tests should be considered as a first suggestion to be applied by user groups of a particular system. Individual users should define other geometries appropriate for specific treatment techniques.

\subsubsection{Individual user tests}

In addition to this general approach, each individual user should define a (sub)set of test situations, which are considered relevant for clinical applications in that particular department.

- This set may be limited to a subset of the tests given, for instance, by AAPM TG 23 [4], the set of tests applied by the NCS [26] and given in Appendix A.4.2.

- The number of points (depths) used in each test situation may be limited.

- The subset should be measured for each available photon beam quality, while some additional verification measurements are recommended for identical machines.

\subsubsection{Comparison of measured and calculated data}

\section{Scope}

To provide tools to compare dose measurements with dose calculations performed by a TPS. 


\section{Background}

For the analysis of a large amount of data, which is common practice in a complete dosimetric verification of a modern TPS, several approaches are possible. Dosimetric verfication may be divided in: checks at points, including absolute dose verification, checks along lines (depth dose curves and beam profiles), checks in planes (isodose lines) or checks in three dimensions ( isodose surfaces and dose-volume histograms). Dose values are often available for points at $1 \mathrm{~cm}$ grids or even smaller grid sizes.

If a study is performed in which the dose calculation in many points of comparable situations is evaluated, in some of these points the tolerance may be exceeded but the overall result may still be quite satisfactory. This may happen when a set of test data such as the AAPM [4] or the NCS [26] test dataset is used to evaluate a new TPS or software release. If the difference between measured and calculated dose in a single point exceeds the tolerance value, this does not necessarily lead to a negative overall result if other comparable points are well within the tolerance. Furthermore, it is not always a simple task to report on the results of an extensive test procedure, containing many data points. The results for very different test situations must then be compared. In these situations the use of statistical methods to support the final conclusions is needed.

\section{Suggestions}

a. The quantity confidence limit has been proposed as an overall quality parameter to compare a set of measured and calculated data (e.g. Venselaar et al. [18]). The confidence limit is based on the average deviation (systematic error) between calculation and measurement for a number of data points in a comparable situation, and the standard deviation (SD ) of the differences (random error). The confidence limit, $\Delta$, can be defined as:

$$
\Delta=\mid \text { average deviation } \mid+1.5 \times \mathrm{SD}
$$

b. For a specific test situation, one can determine the confidence limit and judge the quality of the calculations with only one parameter. The confidence limit can be exceeded because the average deviation of all points is too large, but also in cases where a few data points show extreme deviations and therefore do increase the standard deviation. The factor 1.5 in this expression is based on experience and is shown to be an appropriate choice for clinical practice [26]. A factor $>1.5$ would emphasize the effect of random errors too much, while a factor $<1.5$ would stress the relative importance of systematic deviations. 
c. It should be noted that, when applying the concept of the confidence limit with a value of the multiplication factor of 1.5 , an acceptable outcome of a test may imply that still $6.5 \%$ of the individual data points exceeds the tolerance value for that particular situation.

d. For the comparison of measured and calculated depth dose data or beam profiles, the confidence limit may not always be the best quantity to express differences. In regions with a large dose gradient, an accuracy criterion based on distance, i.e., the distance-toagreement between calculation and measurement, is a better quantity.

e. A function that combines dose and distance discrepancies, the field accuracy, has been proposed by van 't Veld [27] and is expressed in [\%;mm]. The underlying assumption is that in many situations dose and distance criteria are numerically close to each other, e.g., $3 \%$ and $3 \mathrm{~mm}$, which will then result in a field accuracy of $3[\% ; \mathrm{mm}]$.

$\mathrm{f}$. The idea of combining dose-differences and distances-to-agreement between two sets of data has been further explored for two-dimensional dose distributions (e.g. Harms et al. [28] Low et al. [29]). These authors combined both types of deviation in one quantity called gamma index, which was subsequently applied to verify dose distributions in planes. In this way dose distributions, measured for instance with film, can be compared with dose distributions calculated in the film plane, identifying areas where predefined criteria (in \% or $\mathrm{mm}$ ) could not be reached; i.e. where the gamma index was higher than one.

A rapidly increasing number of dose verification tools such as described above is currently becoming commercially available by TPS manufacturers and vendors of treatment verification equipment. Particularly the wide-scale introduction of IMRT has increased the need for such verification methods.

\subsection{Monitor unit calculation}

\subsubsection{MU calculations performed by the TPS}

\section{Scope}

To verify the outcome of MU calculations performed by the TPS with measurements.

\section{Background}

Most treatment planning systems generate both a 3-D dose distribution and the number of MUs for each beam required for a pre-defined dose value at a specified point. The 3-D dose 
distribution is relative to some normalization point, for which there may be a wide range of choices, such as the ICRU dose specification point [30] [31], the centre of the open field, the isocentre, the depth of dose maximum, $d_{\max }$, or a reference depth on the central beam axis, $d_{\text {ref. }}$ Then, the TPS calculates the number of MUs for a given reference dose at the dose normalization point. It is essential for the user of a TPS to understand the principles of the algorithms employed in these MU calculations. For that purpose dose measurements at the dose normalization point can be compared with MU calculations. Sources of deviation between calculation and measurement are: the uncertainties involved in the test measurements; the algorithm and the data used to calculate the relative dose and the computation of the number of MUs.

\section{Suggestions}

The evaluation of the performance of the TPS as discussed in the previous paragraphs will preferably include all steps. Suggestions for tolerances for the accuracy of dose calculations are given in Appendix A.4.1. It should be noted that these values concern the combined error in the monitor unit and relative dose calculation.

In case the TPS fails to meet these accuracy requirements, there are several options to be considered by the user. These include the following:

a. Check the basic beam data entered in the TPS.

b. Check the test beam data set.

c. Adjust the model parameters.

d. Restrict the clinical use of the TPS to geometries that passed the test.

e. Inform the vendor about the findings.

It is the responsibility of the user to decide on the appropriate steps before accepting the TPS for clinical use.

\subsubsection{Independent MU calculation}

\section{Scope}

To verify the outcome of MU calculations performed by the TPS by means of an independent MU calculation method.

\section{Background}

We recommend to use a second independent $\mathrm{MU}$ calculation method to verify the $\mathrm{MU}$ data produced by the TPS. A clinical QA protocol, therefore, should include such an independent calculation for the beams of each treatment plan. This calculation can either be performed by 
hand, e.g. using tables and graphs of machine data, or by using a dedicated computer program. In both cases, the accuracy may be less than that of the MU calculation by the TPS; the method serves only to trace gross errors. In section 4.3.1 the MU calculation algorithm of the TPS is implicitly checked in the test cases by measurement of the point doses. This section discusses the need for verification of the results of $\mathrm{MU}$ calculations in clinical conditions, using patient data.

For many years formalisms have been in use for manual dose calculations for megavoltage photon beams. See for example Supplements 11, 17 and 25 of the British Journal of Radiology (BIR [32] [33] [34]) and Khan [35]. We recommend following the outlines of the calculation methods developed in the ESTRO Booklets 3 and 6 (e.g. Dutreix et al. [36], Mijnheer et al., [37]) and in the previously published Report 12 of NCS ([38]). In the ESTRO and NCS formalisms, basically the following three issues are considered:

- a reference depth of $10 \mathrm{~cm}$ for measurement and calculation of output factors of a megavoltage photon beam;

- separation of the output factor into the collimator and phantom scatter correction factor;

- use of a mini-phantom to measure the collimator scatter correction factor.

In this way the uncertainty involved in the definition of the depth of maximum absorbed dose, $d_{\max }$, as used in the conventional approaches is eliminated. Consequently, the influence of contaminant electrons in the beam is eliminated. Thus, the influence of blocks or "missing tissue" in the beam, non-standard SSDs can be taken into account with more accuracy. A formalism for a MU calculation based on these principles can be implemented in a PC based algorithm and used as an independent check of the outcome of the more sophisticated TPS programs.

\section{Suggested steps}

a. Develop a MU calculation program, either for manual calculation or using a computer program, based on the formalisms given in ESTRO Booklets 3 [36] and 6 [37] or NCS Report 12 [38]. See also Venselaar et al. [39].

b. Include in the program the dependence on depth (using the percentage depth-dose, PDD, or tissue-phantom ratio, TPR), SSD, field size, and preferably taking the collimator exchange effect into account.

c. Take into account the dose variation with field size in case of the presence in the beam of a wedge or a blocking tray by using field size dependent correction factors for the wedge, $k_{w}(d, c)$, for the blocking tray, $k_{o, t}(c)$ or $k_{t}(c)$, and eventually for the presence of a block, 
$\mathrm{k}_{\mathrm{o}, \mathrm{b}}(\mathrm{c})$. In this nomenclature, $\mathrm{d}$ is the depth in the phantom, and $\mathrm{c}$ is the size of the field. (Dutreix et al. [36])

d. For more complex situations involving tissue inhomogeneities, off-axis situations and MLC-shaped fields, more sophisticated algorithms are required. Several groups are currently in the process of developing these algorithms.

Output factors of the beam must be measured in a full scatter phantom ( $\mathrm{fsp}$ ), leading to the output ratio or total scatter correction factor $\mathrm{S}_{\mathrm{cp}}(\mathrm{c})$, and in a mini-phantom $(\mathrm{mp})$, leading to the collimator scatter correction factor $S_{c}(c)$. From these latter two correction factors, the phantom scatter correction factor $S_{p}$ is derived by taking $S_{c p}(c) / S_{c}(c)$. $S_{p}$ data, determined in this way from measurements, may be compared with published data as presented for instance by Storchi and van Gasteren [40].

Table 4.1 summarizes the data that must be measured for setting up and checking the MU calculation process. These include depth-dose data, output factors, wedge factors and tray factors.

\subsection{3. $\mathrm{MU}$ calculations for electron beams}

MU calculations for electron fields are generally checked by direct comparison with measured data. The points to be checked are the $d_{\max }$ points on the central beam axis of the electron beams. For those cases where standard beam inserts are clinically used, tables of output factors should be made available. The influence of small blocks in the fields is usually negligible, except for small fields depending on the energy of the electrons of the beam.

Certain machine types can define arbitrary rectangular field sizes by using a variable trimmer system. For example, fields can be defined with settings of the $X$ and $Y$ trimmers between 2 and $30 \mathrm{~cm}$. A simple approach to be followed in a monitor unit calculation algorithm is to use the assumption that the influences of the $X$ and $Y$ settings on the output factor are independent: $\mathrm{OF}(X, Y)=\mathrm{OF}(X, 10) \times \mathrm{OF}(10, Y)$, where a $10 \times 10 \mathrm{~cm}^{2}$ is the reference. This algorithm can be validated with a limited set of measurements for each beam energy, for each machine type. (e.g. Mills et al. [41]).

Another variable that must be checked carefully for its influence on the dose calculation is the source surface distance. Within the range of SSDs used clinically, e.g. from $95 \mathrm{~cm}$ up to a maximum between $110-120 \mathrm{~cm}$, the inverse square law can be applied. If deviations occur between calculated and measured dose, a virtual source position can be inserted in the algorithm to obtain a proper correction. 
Table 4.1 Summary of measurements, parameters and conditions to obtain the photon beam data required for monitor unit calculations.

\begin{tabular}{|c|c|c|c|c|c|}
\hline Quantity & $\begin{array}{c}\text { Field } \\
\text { description }\end{array}$ & Square fields $\mathbf{s}^{1)}$ & $\begin{array}{l}\text { Rectangular } \\
\text { fields }\end{array}$ & $\begin{array}{c}\text { Source-detector } \\
\text { distance }\end{array}$ & Phantom $^{2}$ \\
\hline \multirow[t]{3}{*}{$P D D, T P R$} & open & + & $t^{3)}$ & 100 & fsp \\
\hline & Wedged & + & $++^{3)}$ & 100 & Fsp \\
\hline & Tray & + & - & 100 and 80 & $\mathrm{mp}$ or fsp \\
\hline \multirow[t]{2}{*}{$S_{c p}$} & Open & + & $+3)$ & 100 & fsp \\
\hline & Wedged & + & $++^{3)}$ & 100 & fsp \\
\hline \multirow[t]{3}{*}{$S_{c}$} & Open & + & $t^{3,4)}$ & 100 & $\mathrm{mp}$ \\
\hline & Wedged & + & $+^{3)}$ & 100 & $\mathrm{mp}$ \\
\hline & Tray & + & - & 100 & $\mathrm{mp}$ \\
\hline$k_{w}(d, c)$ & wedged/open & + & - & 100 & fsp \\
\hline $\begin{array}{c}k_{o, t}(c) \text { or } \\
k_{t}(c)\end{array}$ & tray/open & + & - & 100 & $\mathrm{mp}$ or fsp \\
\hline
\end{tabular}

1. For these measurements the side of the square fields may be set to $4,5,6,7,8,10,12,15,20,25,30,35$ and $40 \mathrm{~cm}$.

2. $\mathrm{fsp}=$ full scatter phantom; $\mathrm{mp}=$ mini-phantom

3. For these sets of measurements a limited number of elongated fields has to be chosen for the purpose of checking the data against published data and/or confirmation of the outcome of calculations; for example, the application of the equivalent square field method.

4. A full set of rectangular fields can be measured, with independent setting of the $X$ - and Y-collimator, e.g., at 4, $5,6,7,8,10,12,15,20,25,30,35$ and $40 \mathrm{~cm}$. Fitting procedures can be applied to limit the number of measurements of rectangular fields. 


\section{Plan evaluation and optimisation}

Conventional treatment planning is an iterative process, starting with the definition of the planning target volume and the organs at risk: the dose defining structures. In the next step, an initial beam set-up is chosen in combination with a set of beam weights. Subsequently, a dose distribution is calculated. Based on the displayed dose distribution in relation to the dose defining structures, the beam set-up parameters will be adjusted and the dose distribution is calculated again. The dose distribution is evaluated once more and this process is continued until an optimum result is obtained. This evaluation usually concerns minimum and maximum target dose and maximum or mean dose to the organs at risk. These parameters may be assessed in various display modes of dose and anatomy (section 5.1) or in graphs of dose versus volume (section 5.2). Besides dose-based optimisation also biology-based parameters may be used (section 5.3).

Correct presentation of the calculated dose distribution in combination with the anatomical structures is of crucial importance in this optimisation process. For example, an erroneous interpolation algorithm in the construction of the 95\% isodose line may easily result in insufficient target coverage, even if the underlying dose distribution was correctly calculated. In this chapter we present a number of tests to verify the accuracy of the plan evaluation tools in the TPS. It is important to note that presented dose values should be compared with calculated dose values and not with the measured dose data. Verification of the agreement between calculated and measured dose values has been discussed in chapter 4 .

\subsection{Dose Display}

\section{Scope}

To ensure that the various dose representations are consistent and displayed correctly in relation to the patient's anatomy.

\section{Background}

Decisions during treatment plan optimisation are often based on the analysis of dose displays in relation to anatomical data. Dose display can be either 0-D: dose points, 1-D: dose profiles, 2-D: isodose lines or 3-D: isodose surfaces. A dose point is a user defined single point in 3-D space in which the dose value is either interpolated between the dose values of grid points or is calculated independently. A dose profile is distribution of dose values along a user defined line which are either interpolated between dose values of a 2-D 
or 3-D dose grid or are calculated independently. Isodose lines are generally constructed from dose values in a 2-D or 3-D dose grid and are used to evaluate the dose distribution in a plane. Isodose surfaces constructed from dose values in a 3-D dose grid and are used to evaluate dose distributions in a volume.

The various types of dose display are best checked on the basis of a user defined 3-D distribution of dose values. Such a procedure has the advantage that any possible deviation has to be the result of an improper display function and is, for example, not affected by inaccuracies in the dose calculation algorithm. It also gives the user the opportunity to see how the dose display tools handle extreme dose distribution. Figure 5.1 shows how a treatment planning system could respond to a 2D block-shaped dose distribution. Special attention should be paid to interpolation of dose values between points on either side of the body contour, since in many TPSs the dose may not be defined outside the patient and is set to zero. Consequently, the dose interpolation between the body contour and the nearest grid points inside the patient is not straightforward in these cases.

Although not always possible, it may be worthwhile to perform the following tests using 3-D dose distributions with known characteristics, created outside the TPS with custom software. The advantage of externally created dose distributions is twofold. Firstly, the relation between position and dose is known beforehand and secondly, custom-made dose distributions can be shaped in any way (e.g. cubical shaped) and are not governed by beam characteristics.

\section{Suggested tests}

a. Dose points

Define a dose point in the various ways possible. Check that the point is defined at the desired 3-D co-ordinates and is displayed at the correct 3-D location. Verify that the dose value is calculated or interpolated consistently and is displayed correctly. If possible compare the display with numerical text output. Check that the dose value is always updated correctly after any change that may affect the calculation outcome. When a dose point is stored, it should be uniquely labelled with a name or number.

b. Dose profiles

Define a dose line in the various ways possible. Check that the begin and end points are defined at the desired 3-D co-ordinates and the line is displayed correctly. Verify that the dose values along the line are interpolated and displayed correctly, if the profile is derived from a dose grid. If possible compare the display with numerical text output. Verify that the dose profiles are plotted correctly with regard to the axes and legends. 
Check that the dose values are always updated correctly after any change that may affect the calculation outcome. When a dose profile is stored, it should be uniquely labelled with a name or number.

c. Isodose lines

Check the correct display of isodose lines in axial, sagittal, coronal and oblique planes. Verify that the isodose lines are consistently displayed at the intersection of differently oriented planes. If the point dose can be displayed by means of a mouse-click, validate that the dose of various points at the isodose lines are consistent with the indicated dose level. Verify that the colour wash display corresponds with the isodose lines and agrees with the point dose display. Check that the isodose lines are always updated correctly after any change that may affect the calculation outcome.

d. Isodose surfaces

Check the correct display of isodose surfaces in 3-D views and that the isodose surfaces are consistent with the isodose lines in various planes (see Fig. 5.2). Verify that isodose surfaces break up in unattached volumes for higher dose values in the cases where isodose lines break up.

\subsection{Dose volume histograms}

\section{Scope}

To ensure that a 3-D dose distribution over a 3-D anatomical structure is accurately represented in a dose volume histogram.

\section{Background}

The dose volume histogram (DVH) is an important plan evaluation tool in 3-D treatment planning. In general, a 3-D dose distribution and a 3-D anatomical structure are needed in order to generate a DVH. Firstly one should verify the agreement of the calculated volume of a given structur with its actual volume. The algorithms used in most TPSs are either based on grid sampling or random sampling. Depending on the algorithm, alignment effects can occur (e.g. Van 't Veld and Bruinvis [42]). Therefore, not only cube-like structures have to be examined, but also (irregular) structures defined by a large number of points, which are less sensitive to grid-based artefacts (e.g., spheres). In addition the accuracy of computed volumes of combinations of structures (using Boolean logic) should be examined. 
Secondly one should verify that the different voxel-dose-elements are distributed over the correct dose bins of the DVH. This requires a well-defined 3-D dose distribution of simple geometry. If the user has access to the file format of the 3-D dose distributions, then the user can directly create a 3-D matrix of dose values.

Finally the user should verify that the calculated DVHs are always updated correctly after any change that affects the calculation outcome, i.e. a change in structure definition, beam set-up or dose calculation.

\subsubsection{Volume computation}

\section{Suggested tests}

\section{a. Structures}

Define various structures with known dimensions: cubes of different sizes and orientations, spheres with different radii. If a sphere is defined in $2 N+1$ equidistant slices (with slice $-N$ and $N$ the tangent planes) and by $P$ points in each slice (see fig 5.3), the volume of the sphere can be computed analytically (see appendix A.5)

b. Volumes

Compare the volumes calculated by the TPS with the exact volumes for the various structures. Vary the number of DVH sampling points.

c. Accuracy

One should require 1\% agreement for irregular and sphere-like structures with more than 1000 (random or grid based) sampling points; 1\% agreement for rectangular structures with more than 1000 random sampling points; 3\% agreement for rectangular structures with more than 1000 grid based sampling points.

d. Composite structures

Define different intersecting structures ( $A$ and $B$ ) of known dimensions and known overlap. Analyse the volumes of $A, B, A \cup B$ and $A \cap B$. Verify that $V_{A}+V_{B}=V_{A \cup B}+V_{A \cap B}$

\subsubsection{Dose binning}

\section{Suggested tests}

a. Created dose grid values 
Define a structure with known dimensions and orientation and a 3-D block-shaped dose distribution with only two dose values. Calculate a differential and cumulative DVH and analyse the result. Recommended accuracy requirement: $1 \%$.

If the user cannot define the dose grid values, the next two tests are recommended

b. Calculated dose grid values (1)

Set-up a $10 \mathrm{~cm} \times 10 \mathrm{~cm}$ square field $(S S D=100 \mathrm{~cm}$ ) and calculate the $3-D$ dose distribution; Define a rectangular structure, around the central beam axis, with $1 \mathrm{~cm}$ length, $1 \mathrm{~cm}$ width and approximately $20 \mathrm{~cm}$ height, starting at depth $d_{\max }$ (see fig 5.4).

c. DVH verification

Calculate a differential and cumulative DVH and compare these with DVHs obtained from the corresponding PDD table. Use a spread sheet programme to generate a frequency distribution with a dose-interval corresponding to the DVH obtained from the TPS. Recommended accuracy requirement: 0.5\%

d. Dose grid values (2)

Define a cylindrical phantom with radius $15 \mathrm{~cm}$ and a rectangular structure of $20 \mathrm{~cm} \times 20$ $\mathrm{cm} \times 5 \mathrm{~cm}$. Set-up a full arc technique with arc length: $0^{\circ}-360^{\circ}$ and field size $10 \mathrm{~cm} \times 10$ $\mathrm{cm}$. Calculate the 3-D dose distribution (Fig. 5.5).

e. DVH verification

Calculate the cumulative DVH of the rectangular structure. Determine the volume at several isodose values. Verify that the volume difference is equal to $\pi \cdot\left(R_{1}{ }^{2}-R_{2}{ }^{2}\right) \cdot d$, where $R_{1}$ and $R_{2}$ the radii of the cylinders with the corresponding isodose lines and $d$ the width of the structure (i.e. $5 \mathrm{~cm}$ ). Recommended accuracy requirement: $\pm 5 \%$

\section{$5.3 \quad N T C P$ and TCP calculations}

\section{Scope}

To ensure that the presented NTCP and TCP values are in agreement with the underlying model

\section{Background}

Modern TPSs often provide estimates of the biological effects of a specific dose distribution based on calculations with normal tissue complication probability (NTCP) and tumor control probability (TCP) models. It should be stressed that these models are theoretical and thus approximate. They require validation with clinical data. If such tools are used for clinical 
treatment planning purposes, it is essential that they are included in the QA program. The input of these models generally consists of a DVH in combination with a number of model parameter values describing the dose response characteristics of a specific organ or tumour.

\section{Suggested tests}

a. Calculate the NTCP-value and TCP-value for an arbitrary DVH in combination with some extreme model parameter values. For the Lyman-Kutcher-Burman model (e.g. Lyman [43], Kutcher [44]), the parameter values listed in Table 5.1 should yield the NTCP-value in the rightmost column. Recommended accuracy requirement: none, except for interpolation effects.

b. Generate a simple DVH with one or two dose bins. Calculate the corresponding NTCPvalue and/or TCP-value. Perform in addition an independent calculation by hand. Check both results. Recommended accuracy requirement: none

\section{$5.4 \quad$ Composite dose distributions}

\section{Scope}

To ensure that dose distributions composed of a number of dose distributions from different treatment plans are correct.

\section{Background}

The TPS may have the possibility to add dose distributions of two or more separate treatment plans. This can be a very useful tool when evaluating the total dose distribution over the entire treatment course. The possibility of subtracting dose distributions is practical for comparing two different treatment plans. Aspects that influence the resulting composite dose distribution are: the dose prescription for each component, the composite and original dose grid spacings, the accuracy of addition/subtraction.

\section{Suggested tests}

a. Set-up the following isocentric techniques: (1) one AP beam with field size $10 \mathrm{~cm} \times 10 \mathrm{~cm}$ and gantry angle $0^{\circ}$, delivering $1 \mathrm{~Gy}$ at the isocentre; (2) one PA beam with field size 10 $\mathrm{cm} \times 10 \mathrm{~cm}$ and gantry angle $180^{\circ}$, delivering $1 \mathrm{~Gy}$ at the isocentre; (3) one AP and one PA beam as in (1) and (2), delivering $2 \mathrm{~Gy}$ at the isocentre. 
b. Verify that the dose of the composite technique of (1) + (2) - (3) equals zero throughout the entire irradiated volume (Fig. 5.6).

c. Repeat this test for different dose grid spacings and types of dose prescriptions (Gy, cGy, $\%)$.

d. Recommended accuracy requirement: $\pm 0.5 \mathrm{cGy}$

Table 5.1: the NTCP-value of a dose volume histogram, as a function of some extreme non-realistic parameters in the Lyman-Kutcher-Burman model. $\mathrm{n}$ is the volume exponent, $\mathrm{m}$ is the slope of the dose effect curve.

\begin{tabular}{llll}
\hline $\mathrm{TD}_{50}$ & $\mathrm{n}$ & $\mathrm{m}$ & $\mathrm{NTCP}$ \\
\hline 0 & - & - & 1 \\
$\infty$ & - & - & 0 \\
$<\mathrm{D}_{\max }$ & 0 & 0 & 1 \\
$>\mathrm{D}_{\max }$ & 0 & 0 & 0 \\
$>\mathrm{D}_{\min }$ & $\infty$ & - & 0 \\
- & - & $\infty$ & 0.5 \\
\hline
\end{tabular}




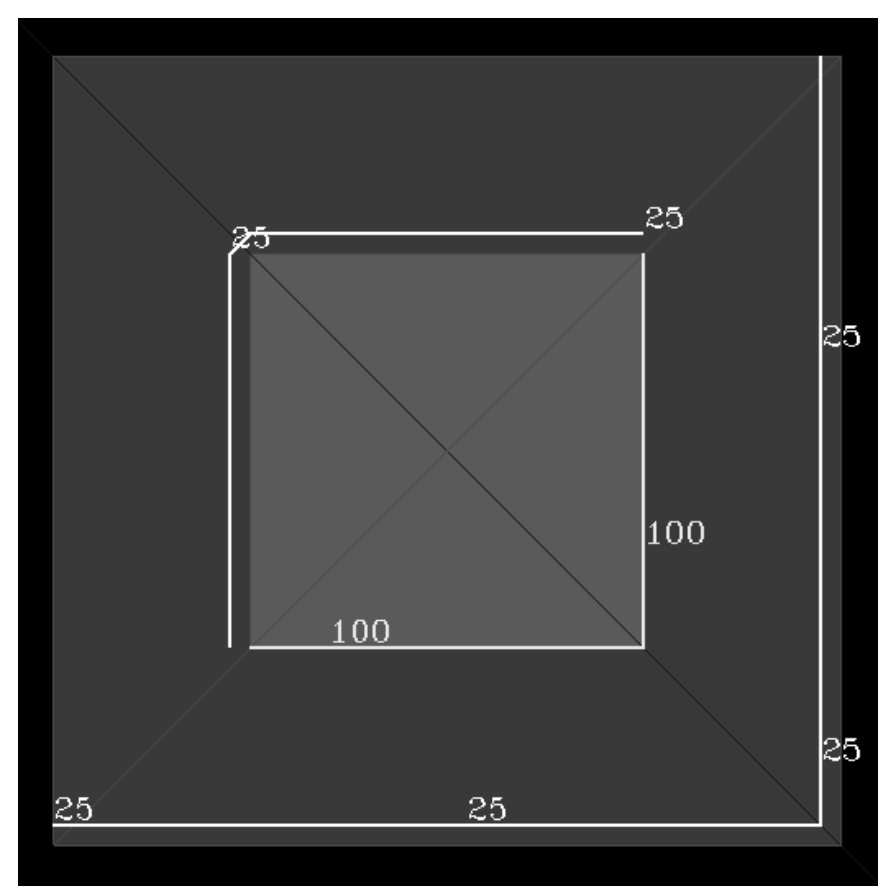

Fig 5.1: Dose distribution showing non-closed isodose lines. The different grey-scale-areas represent different dose areas (0\%=black, 25\%=grey, 100\%=light-grey). 


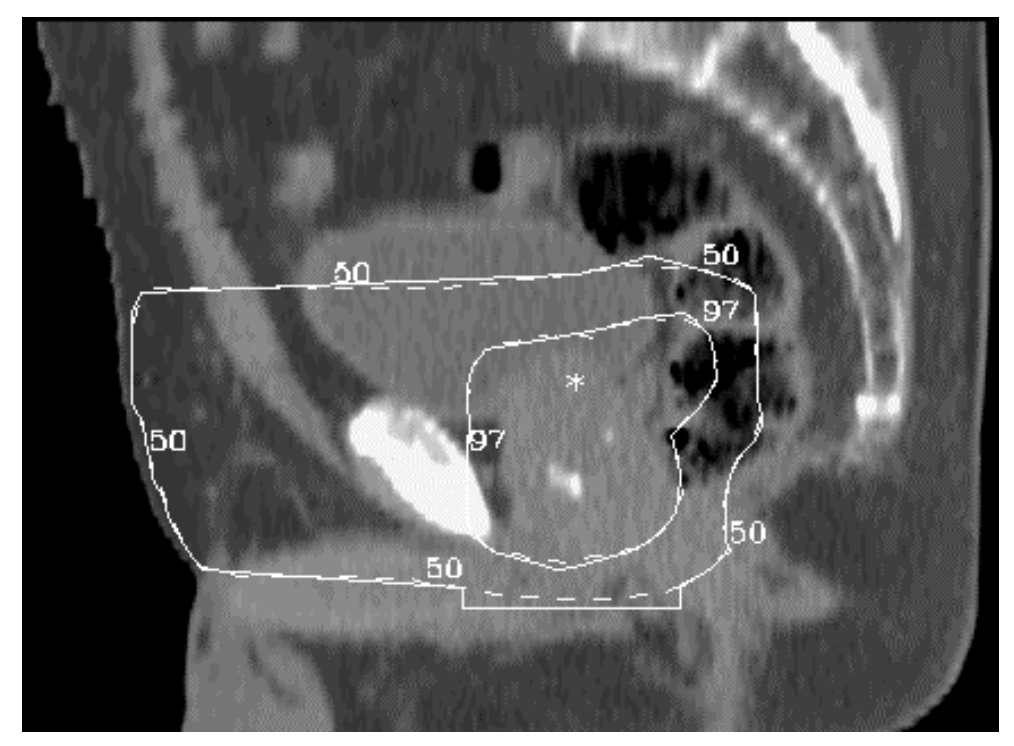

Fig 5.2: Sagittal plane with the original $50 \%$ and $97 \%$ isodose lines (solid) and the sagittal projections of the corresponding isodose surfaces (dashed). In this case, a difference can be noticed at the caudal part of the $50 \%$ isodose. 


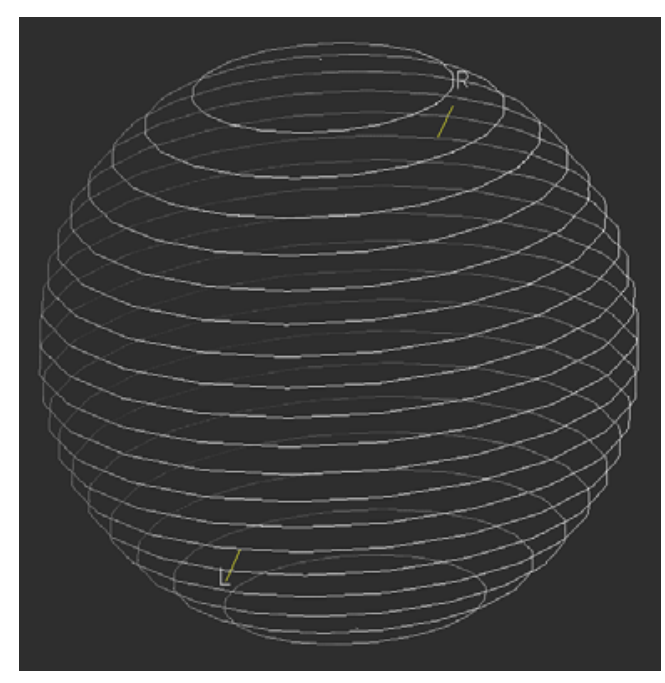

Fig 5.3: Example of a sphere approximation, defined with 20 points in each of the 21 equidistant slices 


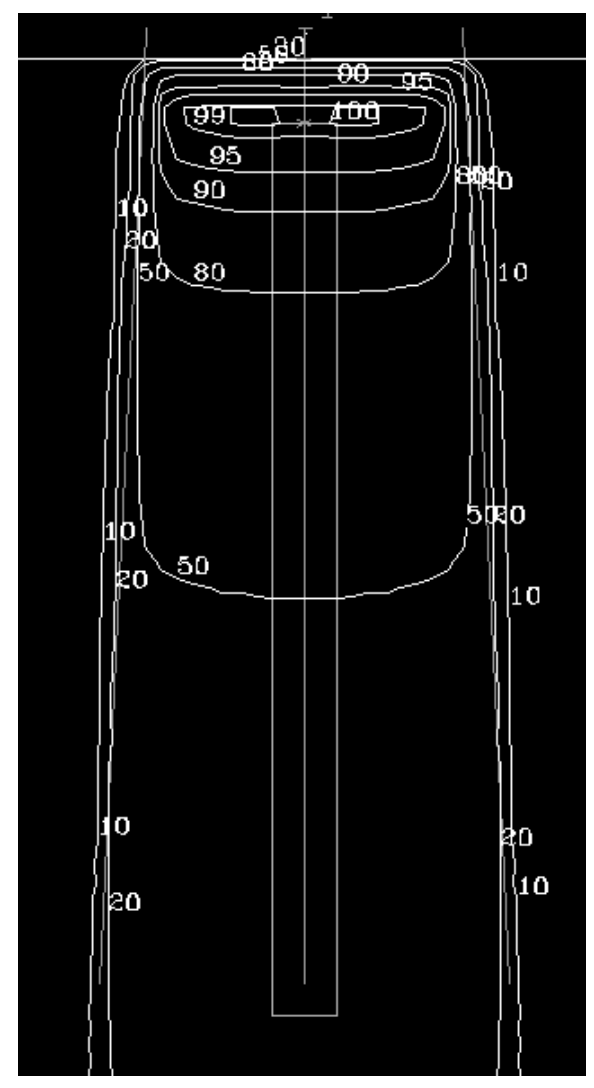

Fig 5.4: Elongated structure defined along the central beam axis 

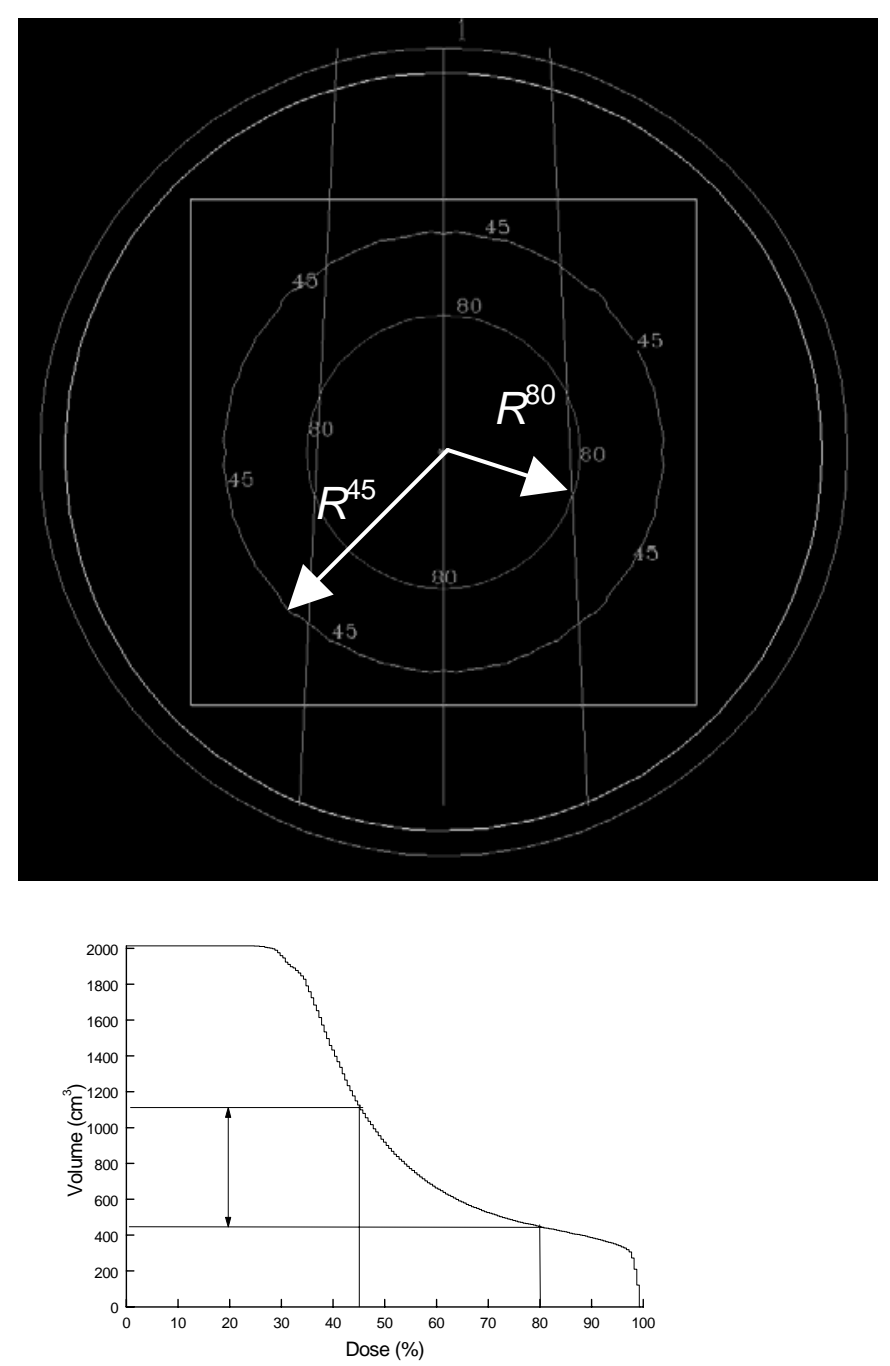

Fig.5.5: Circular dose distribution of an arc technique of a square structure encompassing the $45 \%$ isodose line. The volume between the $80 \%$ isodose line and the $45 \%$ isodose line in the cylinder has to be equal to $\pi\left(R_{45}{ }^{2}-R_{80}{ }^{2}\right)$ d, with $R_{45}$ and $R_{80}$ the radii of the $45 \%$ and $80 \%$ isodose line, respectively, and $d$ the width of the structure 


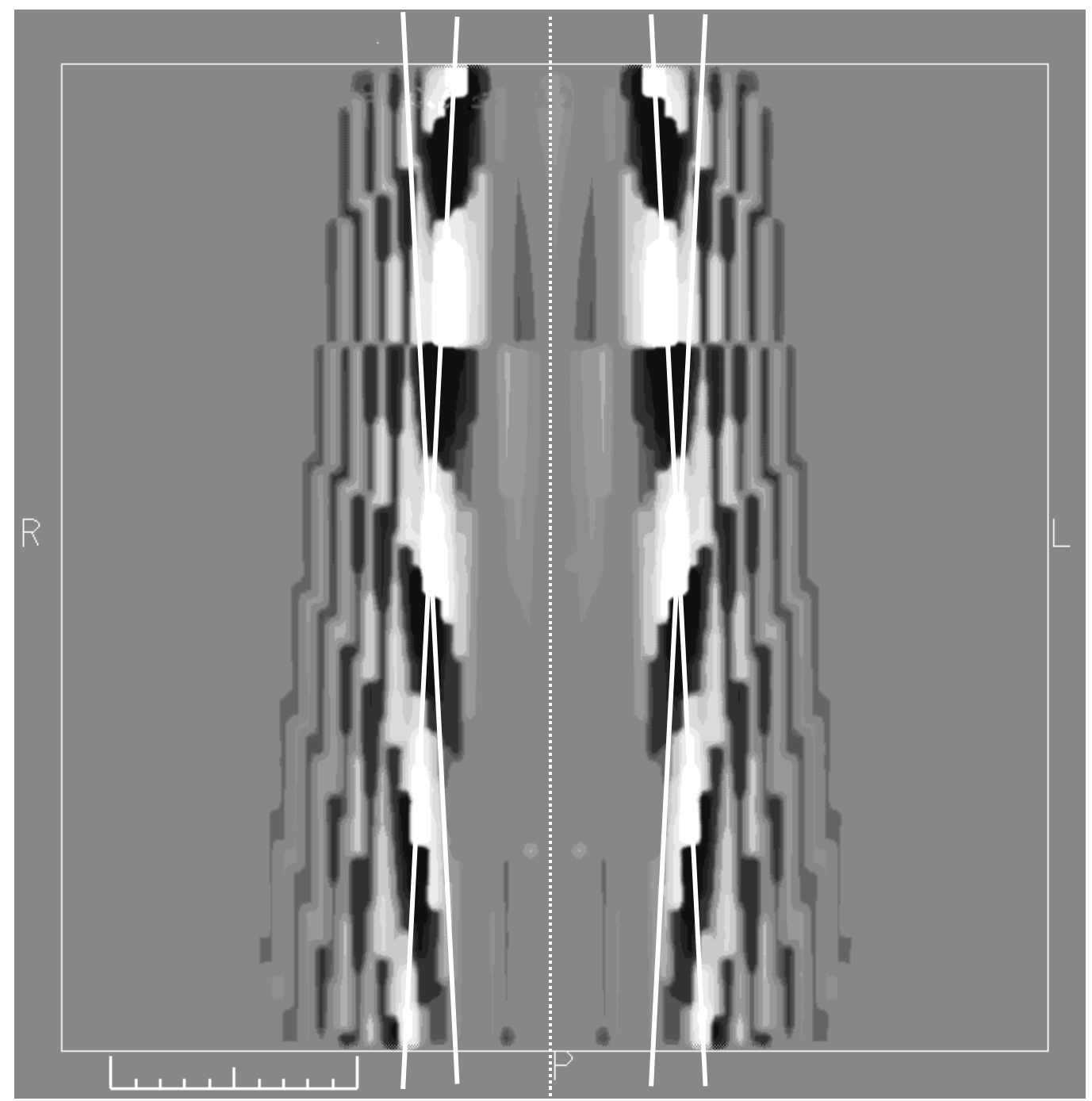

Fig 5.6: Example of an error that could occur in the composite dose distribution. The dark and light grey areas denote positive and negative dose values, respectively. 


\section{Treatment plan description}

After the treatment planning process has been completed on one (or more) of the TPS computer workstations, first of all the final result has to be documented and stored in the TPS database. This documentation should describe the exact treatment plan and all (definite) choices made by the treatment planner should be traceable. The information may be used by a radiation oncologist to evaluate the plan and to decide on approval. The data may also be used by a clinical physicist to carry out quality control procedures. The documentation may be examined, displayed on a computer screen or printed and plotted on paper.

Secondly, the treatment prescription data have to be transferred to the linear accelerator for actual treatment of the patient or maybe first to a simulator for verification purposes. This transfer may be carried out via printed documentation, but more and more direct electronic data transfer is used. The data may also be transferred to other computer systems of e.g. a block cutting machine or an electronic portal imaging system.

Finally, the treatment plan has to be archived for future clinical or research use. For all purposes the data in this treatment plan documentation should correspond exactly to the data of the plan as generated on the TPS. The information should be clear, unambiguous and complete. The data should be uniquely linked to the specific treatment plan and to the specific patient.

\subsection{Print and plot output}

\section{Scope}

To ensure a complete and correct description on hardcopy of a treatment plan for evaluation, quality control and implementation on the treatment machine

\section{Background}

In general the output consists of a printed list of treatment technique parameters, dose prescription and dose calculation aspects that determine the actual treatment plan. This list of alphanumerical data will also specify what patient anatomy data is used; results of NTCP and TCP computations may also be included. The TPS will further produce graphical data of geometrical and dosimetric aspects of the treatment plan such as plots with isodose lines, DVHs, BEVs, DRRs, 3-D views of patient anatomy, beam set-up and dose distributions. One may distinguish treatment plan prescription and treatment plan evaluation data; the suggested tests describe the minimum of information that is required. 


\subsubsection{Treatment plan prescription data}

\section{Suggested tests}

a. General

Check consistency of: patient name, patient identification (ID), plan ID (name or number), date and time of plan, ID of treatment planner, TPS software version ID.

b. Plan setup

Check consistency of: patient data set ID (used for calculations), type of density correction (voxel based or bulk), parameters of dose grid geometry, patient position (i.e. prone, supine).

c. Dose prescription

Check consistency of: dose per fraction, number of fractions or total dose, fractions per week, coordinates dose prescription point (plan normalization point); beam weight values and points.

d. Beam set-up

Check consistency for each beam of: treatment machine ID, beam ID, radiation modality, energy; isocentre coordinates, gantry, collimator and table angle, SSD; jaw settings $\left(X_{1}\right.$, $\mathrm{X}_{2}, \mathrm{Y}_{1}, \mathrm{Y}_{2}$ ), MLC settings, type and ID of blocks; wedge name or angle, wedge orientation, bolus, compensator.

e. Dose / MU calculation

Check consistency of: date and time of dose calculation, dose calculation algorithm used, calculation parameters (e.g. trayfactor, phantom scatter factor, collimator scatter factor, equivalent field size), number of MUs.

\subsubsection{Treatment plan evaluation data}

\section{Suggested tests}

a. General

Check consistency of: patient name, patient ID, plan ID, date and time of plan.

b. Geometry

Check in BEV and DRR plots consistency of: beam ID, orientation of beam, orientation of patient, scaling of plot.

c. Dosimetry 
Check in isodose and 3-D view plots consistency of: date and time of dose calculation, orientation of patient, scaling of plot.

\subsection{Electronic data export}

\section{Scope}

To ensure a complete and correct description of a treatment plan in an electronic transfer format for processing by another computer system and correct transfer of the file.

\section{Background}

Electronic transfer of treatment plan information to various other computer systems is often required. These systems include among others: another TPS, software programs used for block cutting, bolus or compensator manufacturing, treatment machine control software, record and verify system, electronic portal image system. For each of these systems the required information will be different, but we will assume for simplicity that the TPS writes out one type of format with alphanumerical data. For all data transfer paths the user should be certain that the TPS writes out the required information and that this information is correct. The user should verify that the data is received and read into the other system correctly; although this actually exceeds the concern of TPS QA. Any exported electronic file, used for purposes outside the TPS, should be checked that a one to one relationship between the patient specific treatment plan and the file exists.

\subsubsection{Treatment plan prescription data}

\section{Suggested tests}

a. General Check consistency of: patient name, patient ID, plan ID, date and time of plan.

b. Plan setup

Check consistency of: patient position (e.g. prone, supine, head first, feet first).

c. Dose prescription

Check consistency of: dose per fraction, number of fractions or total dose, fractions per week. 
d. Beam set-up

Check consistency for each beam of: treatment machine ID, beam ID, radiation modality, energy; gantry, collimator and table angle; jaw settings $\left(X_{1}, X_{2}, Y_{1}, Y_{2}\right)$, MLC settings, type and ID of blocks; wedge name or angle, wedge orientation, bolus, compensator.

e. Dose / MU calculation

Check consistency of: date and time of dose calculation, number of MUs.

\subsubsection{Treatment plan evaluation data}

\section{Suggested tests}

a. General

Check consistency of: patient name, patient ID, plan ID, date and time of plan.

b. Geometry

Check in files with BEV and DRR images consistency of: beam ID, orientation of beam, orientation of patient.

\subsection{Treatment plan archiving}

\section{Scope}

To ensure a complete and correct description of a treatment plan in a set of electronic files for archiving in a patient database.

\section{Background}

After the treatment of the patient is completed the information on the treatment plan should be stored as part of the documentation of the treatment, for possible other future treatments, for clinical evaluation of type of treatments or scientific research. The user should verify that the TPS writes out all relevant information in electronic files, that this information is correct and that after retrieval of the stored files the information is read in correctly by the TPS.

\subsubsection{Treatment plan prescription data}

\section{Suggested tests}

a. General

Check consistency of: patient name, patient identification (ID), plan ID (name or number), date and time of plan, ID of treatment planner, TPS software version ID. 
b. Plan setup

Check consistency of: patient data set ID (used for calculations), type of density correction (voxel based or bulk), parameters of dose grid geometry, patient position (i.e. prone, supine).

c. Dose prescription

Check consistency of: dose per fraction, number of fractions or total dose, fractions per week, coordinates dose prescription point (plan normalization point); beam weight values and points.

d. Beam set-up

Check consistency for each beam of: treatment machine ID, beam ID, radiation modality, energy; isocentre coordinates, gantry, collimator and table angle, SSD; jaw settings $\left(\mathrm{X}_{1}\right.$, $X_{2}, Y_{1}, Y_{2}$ ), MLC settings, type and ID of blocks; wedge name or angle, wedge orientation, bolus, compensator.

e. Dose / MU calculation

Check consistency of: date and time of dose calculation, dose calculation algorithm used, calculation parameters (e.g. trayfactor, phantom scatter factor, collimator scatter factor, equivalent field size), number of MUs.

\subsubsection{Treatment plan evaluation data}

\section{Suggested tests}

a. General

Check consistency of: patient name, patient ID, plan ID, date and time of plan.

b. Geometry

Check in BEV and DRR plots consistency of: beam ID, orientation of beam, orientation of patient, scaling of plot.

c. Dosimetry

Check in isodose and 3-D view plots consistency of: date and time of dose calculation, orientation of patient, scaling of plot. 


\section{Periodic quality control}

In chapters 2 to 6 the various functions of a TPS have been discussed and tests have been suggested to cover the initial quality assurance of a new TPS or software version. If the results of such tests are satisfactory, the system will be released for clinical use and treatment plans will be produced every day. Due to the enormous increase in computing power of the TPSs in combination with the technological advancement in diagnostic and therapy equipment, the designed treatment techniques have in many cases become rather complex. With this increase in complexity, unfortunately, it has become much more difficult for the treatment planner or technologist to detect geometric or dosimetric errors after the treatment planning process. This means that one must be able to rely completely on the outcome of the TPS; it therefore has become very important to verify the correct performance of the TPS periodically. Malfunctioning computer hardware, unintended changes in program executables or in data files may cause small or large errors in the results for an individual patient or a group of patients.

\subsection{Treatment planning workstation}

Treatment planning will be performed on some type of workstation, consisting of computer hardware, system software, program software and various data files. Program software and data files may all be stored on the same computer, but may also be located on a central server. To ensure correct performance of the complete system one must verify the correct functioning of the workstation computer, the server computer, the communication between the two systems, the integrity of the program software executables, the integrity of the data files and finally that all these parts function correctly together. This may be verified by tests performed on the individual components and by tests on the complete configuration.

\subsubsection{Computer system}

\section{Scope}

To ensure proper functioning of the workstation. 


\section{Background}

This aspect is not specific for a TPS, so any testing method of computer hardware may be applied. A serious hardware failure will be most likely detected by the user because of distinct malfunctioning of the system, but small problems may require a very sensitive method to be found. We therefore recommend systematic internal checks.

\section{Suggested test}

a. Run a software program that performs a set of mathematical operations that constitutes a heavy workload on the central processor.

b. This program should be executed automatically every night and the results of the operations should always be identical.

c. If any difference in result a warning message should be sent to all users that might log in to the system the next day.

\subsubsection{Program software and data files}

\section{Scope}

To ensure the integrity of all files used by the TPS that contain program software, system configuration parameters and beam data.

\section{Background}

Once the TPS has been commissioned it is very unlikely that the contents of files changes spontaneously. However, changes may be made to a file a by some user by mistake or a file is copied to the wrong directory. Good system security will reduce such chances to a minimum, but one should not rely on that. A so-called checksum test consists of one or more mathematical operations on the data in a file and yields one or more exact numbers. Any change in a file will cause a different number as outcome.

\section{Suggested test}

a. Perform a checksum test on all software and data files. Each file or dataset must have an individual checksum, so that any changes can easily be traced.

b. This test should be performed automatically every night and the results should always be identical.

c. If any difference in result a warning message should be sent to all users that might log in to the system the next day. 


\subsubsection{Complete workstation}

\section{Scope}

To ensure proper functioning of the complete configuration of computer hardware, system software, program software and various data files.

\section{Background}

Correct functioning of the workstation computer, integrity of the program software executables and integrity of the data files in principle does not guarantee correct performance of the complete system. The latter entails that the software program running on the workstation reads the input files, receives user commands, performs computations and produces output files, all in the correct way. We therefore recommend adding to the tests suggested above some clinical user tests, for example a set of standard treatment plans.

\section{Suggested test}

a. Define a number of treatment planning cases that represent the range of clinical applications of the TPS and that use different data files as input. Archive these cases, i.e. patient data and treatment technique, as a standard plan and store the output files that document the dose computations.

b. For each test retrieve an archived case, perform the dose calculations and compare the output files with the stored ones.

c. Perform such a test weekly; the results should always be identical.

\subsection{Data input and output devices}

Patient CT or contour data and field or block shape data may be entered in the TPS by electronic file via a computer network, cdrom, optical disk, floppy disk or magnetic tape. Such data may also be entered on radiographic film or paper plot via a digitiser or film scanner. The TPS may export field, block or MLC shape data and dose distribution data by electronic file or paper plot. Malfunctioning of the data transfer or of the devices may result in errors in the treatment plan and therefore some program of regular quality assurance is required. 


\subsubsection{Peripheral equipment}

\section{Scope}

To ensure proper functioning of separate computer equipment for data input and output of the TPS

\section{Background}

The digitiser, plotter, printers and film scanner should be regularly checked to ensure its function, scaling and linear accuracy. For example, an erroneous scaling factor of the plotter or printer may yield incorrect Cerrobend blocks if the output of the plotter or printer is used as an input for the block cutter device. Similarly, geometrical inaccuracies in the output of the digitiser tablet may result in an erroneous anatomical description of he patient in the TPS.

\section{Suggested test}

a. In section 2.2.2 initial tests are described on e.g. digitiser input. Select one, repeat this periodically and check the consistency of results.

b. Perform such a test every month; the results should be within $1 \mathrm{~mm}$ with the initial contour for the combined check result.

\subsubsection{Transfer of CT data and body contours to the TPS}

Scope

To ensure correct transfer of CT data and body contours to the TPS

\section{Background}

The CT data transfer and that of body contour devices to the TPS should be regularly checked on geometrical accuracy and CT numbers. Geometrical distortions may result in improper target volume delineation, while inaccuracies in CT numbers may yield inappropriate dose distributions.

\section{Suggested test}

a. In sections 2.2.1 and 2.2.2 initial tests are described on CT data and contour input. Select one, repeat this periodically and check the consistency of results.

b. Perform such a test every month; the results should be within $1 \mathrm{~mm}$ with the original data.

\subsection{Display systems}


Errors in the delineation of target volumes and/or critical structures may seriously influence the outcome of the treatment. Coarse structures are delineated based on subtle differences in contrast with the surrounding tissue. It is therefore essential that the display system used by the radiation oncologist provides optimal contrast resolution, whereas the spatial resolution of the display system is generally of less importance.

\section{Scope}

To ensure proper functioning of the display system.

\section{Background}

In this paragraph, one simple test is described which can be used to roughly estimate the contrast performance of the soft-copy display. For more elaborated tests, we would like to refer to DICOM Part 14: "Grayscale Standard Display Function" (http://medical.nema.org ). This document provides examples of methods for measuring the number of just-noticeabledifferences (JNDs) in contrast by the human eye of a specific display system for any range of driving levels.

\section{Suggested test}

a. Display the SMPTE (Society of Motion Picture and Television Engineers) test pattern on the display system. Unauthorised versions of the image can be found on the internet (for example: http://brighamrad.harvard.edu/research/topics/vispercep/tutorial.html).

b. All 11 levels of $0 \%$ to $100 \%$ contrast should be clearly distinguishable. Both the $5 \%$ and $95 \%$ contrast levels should be visible in the $0 \%$ and $100 \%$ contrast levels, respectively.

c. Perform this test every 12 months. 


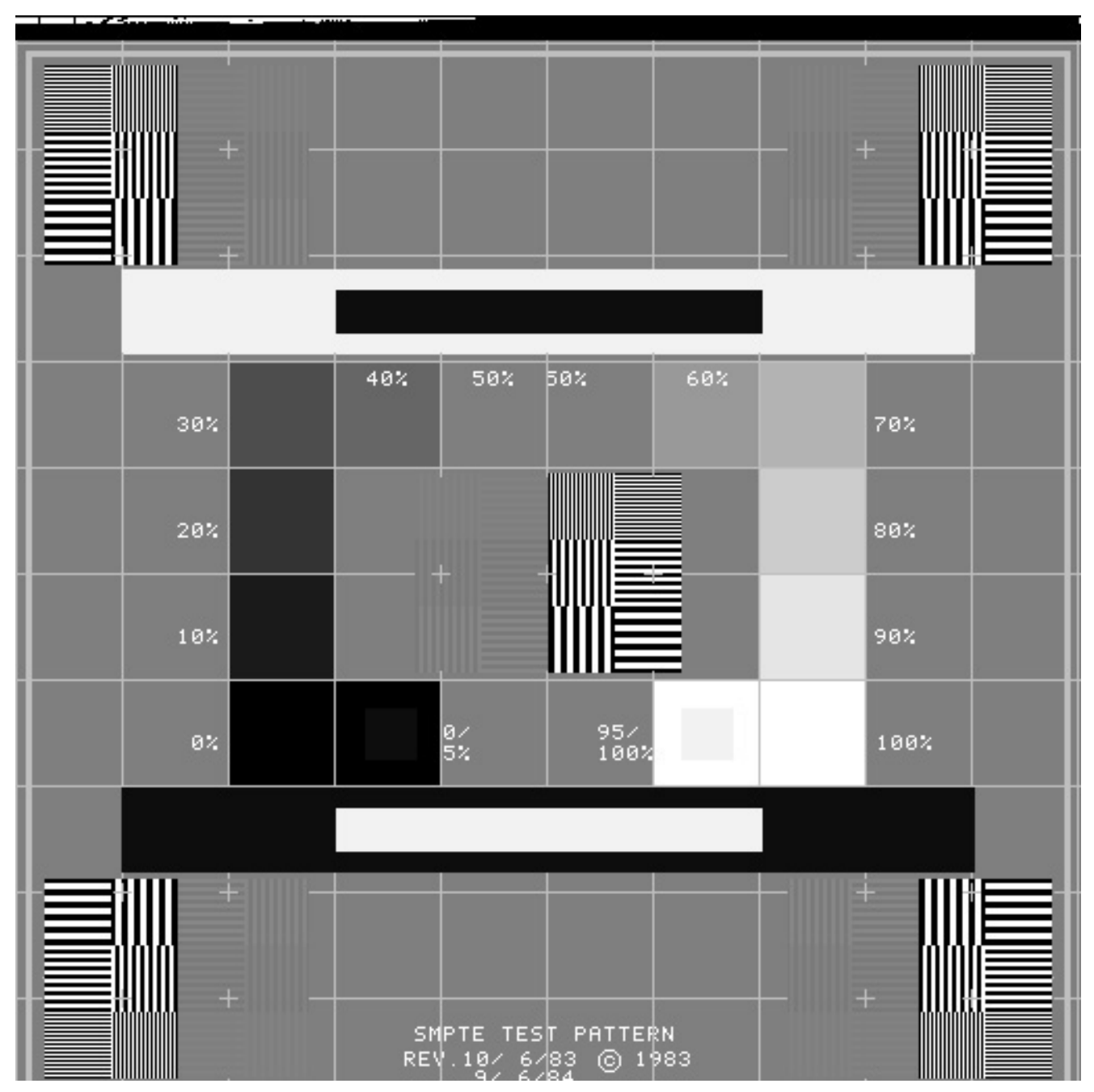

Fig 7.1. The SMPTE test pattern. 


\section{System management and security}

System management is a set of regular activities to ensure the integrity and proper technical performance of both the computer hardware and software. Functional tests, like algorithm verification, are not part of such procedures. System management is crucial for a safe and reliable access to the TPS. It can only be performed adequately if sufficient tools are available and are used by the responsible personnel at sufficient frequency. Detailed knowledge of system components is often neither necessary nor available, therefore system management should aim at checking overall behaviour and indicating need for further expert intervention.

The management of a TPS can be divided into management of the computer systems, software, and network and the system security. A strict distinction between these items is often not possible, however.

\subsection{Computer systems}

\section{Scope}

To ensure proper functioning of the computer hardware.

\section{Background}

Improper hardware functioning can cause errors in treatment planning. Failures that inhibit any use of a hardware piece are obvious; small changes are much more difficult to detect, however, and are thus potentially more dangerous. Computer system management should therefore not only concentrate on preventing major failures but also should aim at detection of changes that are not easily noticed during commissioning, periodic quality control (QC) and regular use.

\section{Suggested tests}

a. Execute the periodic QC program, as discussed in chapter 7, on all system hardware, on both the clinical and research systems.

b. Perform diagnostic tests as supplied by the vendor of hardware pieces as well as hardware intensive calculation and memory checks; they might reveal errors not found in regular QC tests.

c. Consider systematic rewinding and copying of archive tapes e.g., once every 5 years.

d. Check periodically the accessibility of the archive media for both system and data software. 
e. An uninterrupted power supply (UPS) system can prevent interference by other hospital equipment; its proper functioning is tested by switching the input power off.

\subsection{Computer Software}

\section{Scope}

To ensure proper functioning of the computer software.

\section{Background}

The data (software) of a TPS usually consist of at least operating software, TPS executables, site specific procedures, as well as beam, patient and test data. Some parts should only change after an upgrade, other parts after deliberate modification or as a result of regular clinical use. Methods to check proper behaviour include performing a QC program, maintenance of log files, monitoring of trends in system performance.

\section{Suggested tests}

a. Perform the relevant part of the QC program, e.g. the checksum routine, as described in chapter 7, after any deliberate modification made. Because customisation of a TPS is an ongoing process, the checksum procedure should distinguish between the intended modifications and potential unexpected changes in the system.

b. Test site-specific computerised procedures (script files, indirect command files etc.) Less user-friendly functions, commonly independent of the TPS software, are prone to error, e.g. copy and archive of files. This risk can be minimized by streamlining procedures. Such procedures are often site-specific, and thus require extra attention in testing

c. Check available storage resources and keep track of trends (processing speed etc). Unexpected lack of disk or database space might corrupt patient data.

d. Check the contents and relevance of the information in log files.

e. Let the TPS mail a message to alert the responsible personnel when unexpected situations have been encountered, e.g. when a checksum result has changed. Create such a situation and check for mail.

f. Check whether clearing of invalidated patient data is performed completely: try to retrieve such data using name, birth date or patient ID.

g. If available, use a verify (compare) option when critical files are copied, backed-up or restored. 


\subsection{Computer network}

Scope

To ensure proper functioning of the computer network.

\section{Background}

Present network protocols have a lot of inherent transfer verification. It is very unlikely that a network is malfunctioning. Most important tests for a TPS that uses network connections are therefore integrity of files and network behaviour under heavy load.

\section{Suggested tests}

a. Check the integrity of transfer of all types of files sent over the network. Where possible this can best be tested by sending back files and comparing these to the original files.

b. Check system or application performance under maximum realistic network load. Image transfers and login procedures require much network capacity.

\subsection{System security}

\section{Scope}

To ensure a reasonable level of system security.

\section{Background}

System security is that part of system management that should guarantee that personnel can access and modify only those parts of the TPS that are relevant for the tasks to be done whereas any unintended modification is prevented and/or detected. Full security hampers flexible use, so a compromise must be found. Measures should be taken to ensure the use of the latest validated version of both the system and the TPS software.

\section{Suggested tests}

a. Test user privileges by trying to perform actions for various types of users which they should not be allowed to perform, e.g. access to specific directories and files or functionality.

b. Check that different passwords are required to modify TPS files or beam descriptions.

c. Test network access by trying to access the TPS via unintended pathways, e.g., using the departmental PC-network. 
d. Verify that the version of the TPS software is explicitly indicated on any of the output results (prints, plots etc.).

e. Test automatic logout after a period of inactivity, if available. 


\section{Appendices}

\section{A.2.1 CT number representation}

In the physics context CT-scans provide information on the attenuation of radiation in tissues. The attenuation coefficients depend on the electron density $\rho_{\mathrm{e}}$, atomic number $Z$ and beam quality used in the CT-scanner. CT density information is usually given in terms of Hounsfield Units $(\mathrm{HU})$, which expresses the relative attenuation of a tissue, with coefficient $\mu$, with respect to that of water (with coefficient $\mu_{\mathrm{w}}$ ). For an idealized monochromatic X-ray beam, the Hounsfield Unit can be defined as (Huizenga [45])

$$
\mathrm{HU}=1000 \cdot\left(\mu / \mu_{\mathrm{w}}-1\right) \text {. }
$$

In order to use CT density information in for dose calculations in a TPS, a conversion from $\mathrm{HU}$ value to relative electron density (RED) is necessary.

A HU-RED conversion function may be determined by scanning a phantom with known compositions and electron densities of the body and the inserts (e.g. the RMI 465 phantom). We recommend to scan the phantom with different scan protocols (e.g. abdomen, thorax and head \& neck). The CT images are then transferred into the TPS and by taking samples in regions of the body and the inserts, RED values may be determined as a function of $H U$. The relationship between $\mathrm{HU}$ and RED is nonlinear, because of the change in $Z$, going from soft tissues $(-1000 \leq H U \leq 100)$ to more bone-like tissues ( $H U \geq 200)$.

The conversion function from HU to RED may be affected by the choice of scan protocol, i.e. different reconstruction filters, and by different voltages of the X-ray tube. Figures A.2.1.1 and A.2.1.2 show, for a specific CT-scanner, an example of the effect of different reconstruction filters and different voltage values on the the HU values, respectively. Clearly, in this case, the choice of a different reconstruction filter has only a minor effect on the HU values. A change in voltage, however, has a large effect on the HU values, especially for bone-like tissues. If only one conversion function is used, independent of tube voltage, this will result in a variation in RED values, clearly an artefact.

$\mathrm{HU}$ values may be converted into RED using the conversion formulas as given by Thomas [46]. For tissues that have a low $Z$ value, the conversion from RED to HU is given by

$$
\text { RED }=1+\mathrm{HU} / 1000 \text {, for low } Z(-1000 \leq \mathrm{HU} \leq 100)
$$

Bone-like tissues have a higher $\mathrm{Z}$ and the conversion is then given by:

$$
\text { RED }=1+H U / 1950 \text {, for high } Z(H U \geq 200) \text {. }
$$




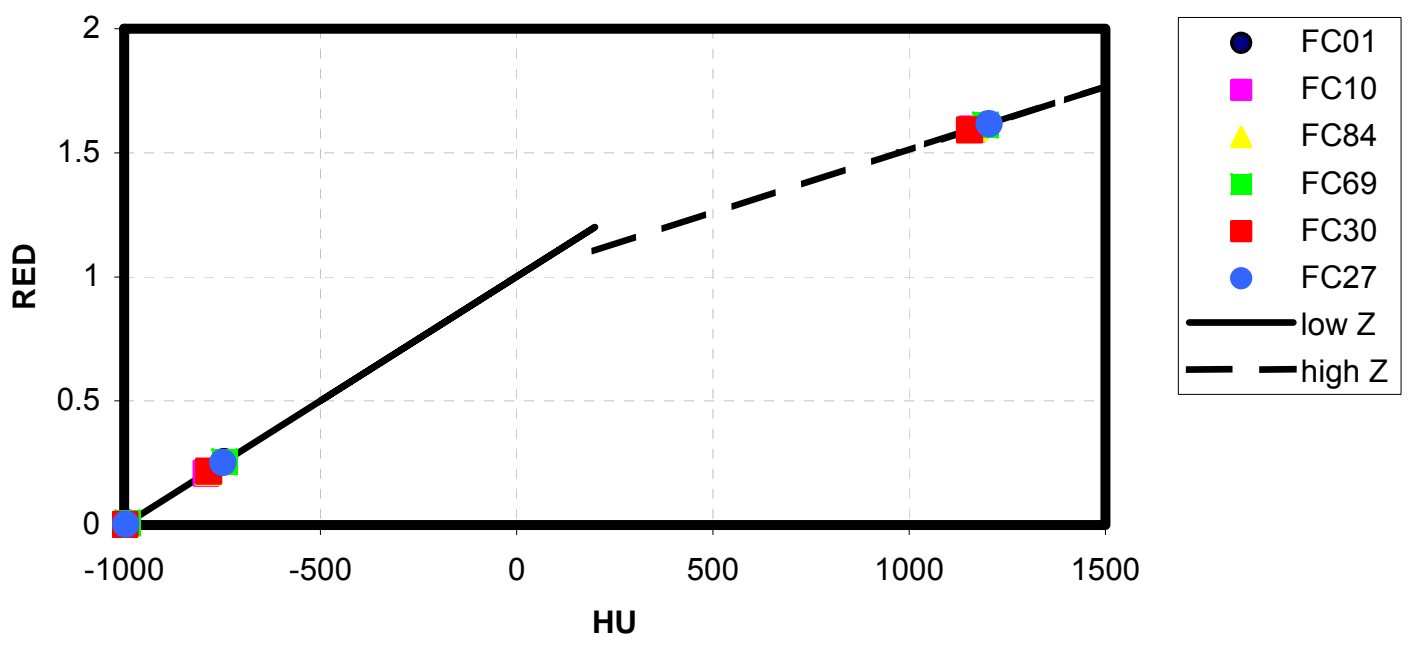

Fig. A.2.1.1: Relationship between HU and RED, for different reconstruction filters for lung (low $Z$ ) and bone-like (high Z) tissues. Measurements were performed on a Toshiba Aquilion scanner; the fixed scan parameters were $120 \mathrm{kV}, 250 \mathrm{~mA}$, 1s, spiral $5 \mathrm{~mm}, 0.854 \mathrm{~mm}$ x $0.854 \mathrm{~mm}$.

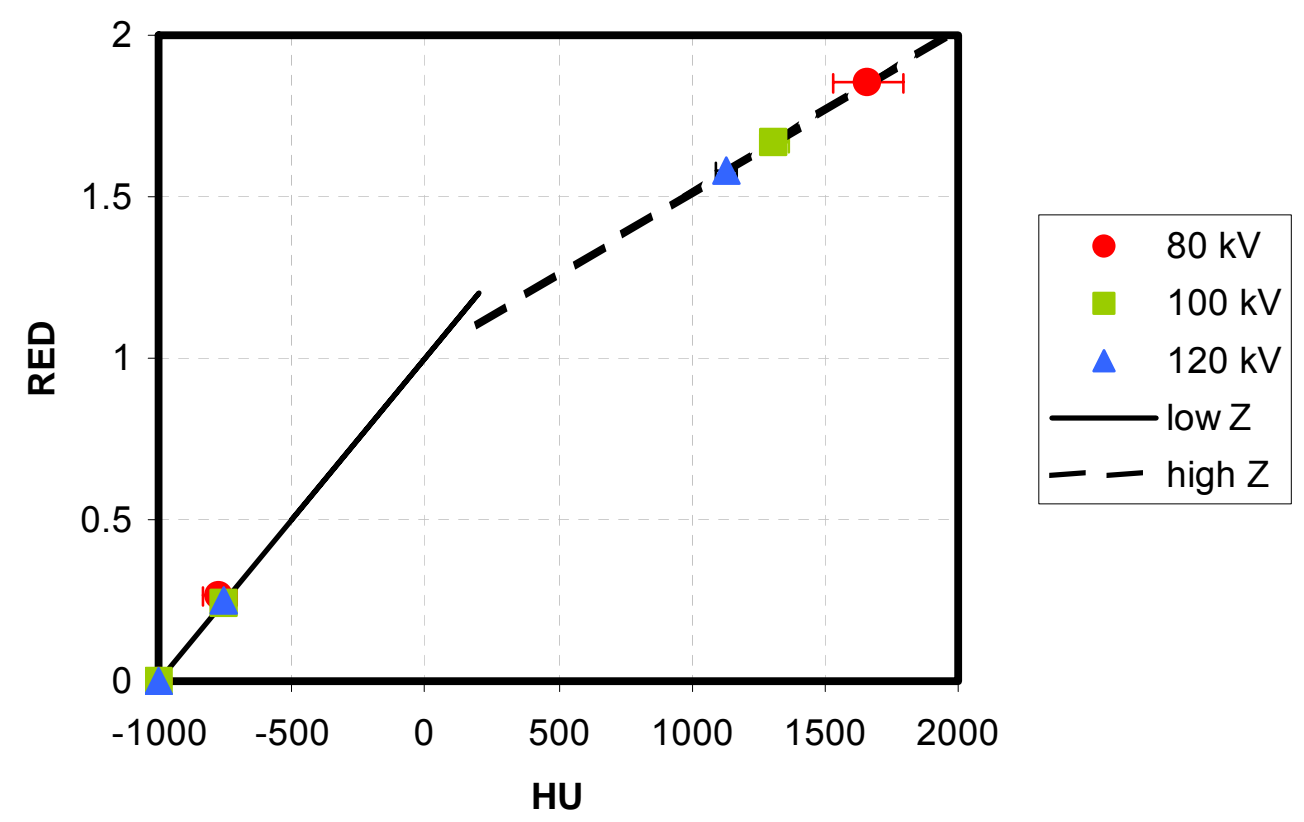

Fig. A.2.1.2: Relationship between HU and RED, for different $X$-ray tube voltages for lung (low $Z$ ) and bone-like (high Z) tissues. Measurements were performed on a Toshiba Aquilion scanner; the fixed scan parameters were FC01 filter, 1s, spiral $5 \mathrm{~mm}, 0.854 \mathrm{~mm} \times 0.854 \mathrm{~mm}$. 


\section{A.2.2 Example of phantom}

The CT-scan test phantom is made of polyethylene (approximating soft tissue); shape and dimensions resemble the abdomen part of body. The width is about $32 \mathrm{~cm}$, the height $22 \mathrm{~cm}$ and thickness is $11 \mathrm{~cm}$. Four cylindrical holes of different diameter and $11 \mathrm{~cm}$ length have been drilled. The one down-left (diameter $4 \mathrm{~cm}$ ) is filled with bone-equivalent material (white circle), the one in the centre (diameter $4.5 \mathrm{~cm}$ ) is filled with cork, resembling lung tissue (black circle). The two others (diameters $3 \mathrm{~cm}$ and $4 \mathrm{~cm}$ ) are filled with different plastics. These structures and the phantom itself can be used to check automated and manual contouring with the TPS. Two small holes $(0.5 \mathrm{~cm}$ diameter, $11 \mathrm{~cm}$ length), $24 \mathrm{~cm}$ apart, left and right, and two similar holes $15 \mathrm{~cm}$ apart, top and bottom, can be used to verify correct horizontal en vertical dimensions. Checks of surface computation and contour extraction can also be performed.

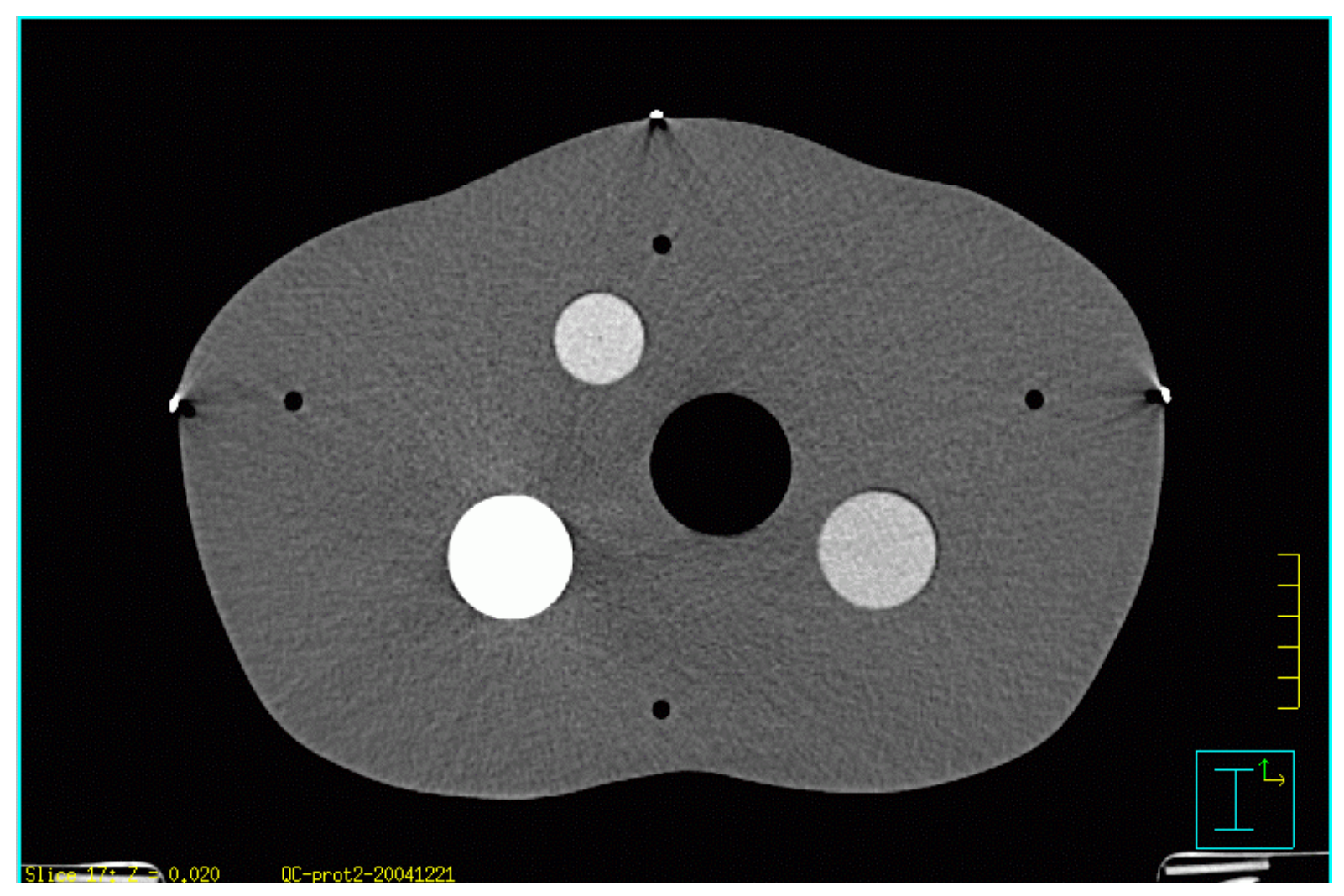

Fig. A.2.2.1: CT-scan axial slice of "abdomen phantom". 


\section{A.2.3 Test of 3-D expansion algorithm}

To test the 3-D expansion algorithm of a TPS, a geometrical structure known as "diabolo" may be defined. An example is shown in figure A.2.3.1. The geometry of this structure is defined by one contour per slice and expanded in three dimensions. The accuracy of the algorithm can be assessed by comparing the generated 3-D margin with the predicted margin. For this purpose axial, sagittal and coronal slices can be looked at. For both the "diabolo" shape and the 3-D margin mathematical expressions are given in this appendix and values of simulation parameters are suggested.

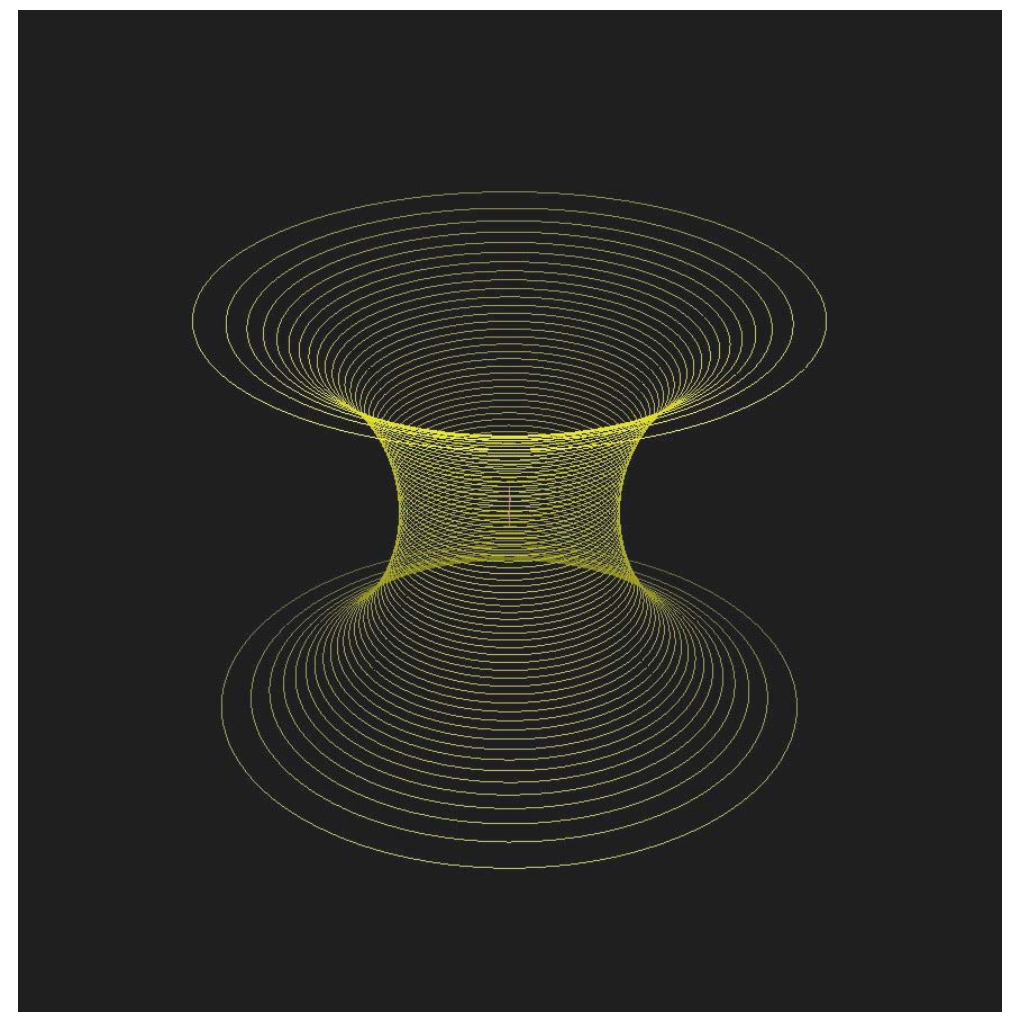

Figure A.2.3.1. "Diabolo" structure.

The "diabolo" structure has circular symmetry around one axis; here the $y$-axis which is the axis in cranio-caudal direction. The structure is thus described by circles with radius $R_{1}$ per $y$ position, $R_{1}(y)$. The length of the radius $R_{1}(y)$ is defined by the circumference of a circle with radius $R_{2}$ as shown in figure 2 . The following equations are now valid: 


$$
\left\{\begin{array}{c}
x_{C}^{2}(y)+y^{2}=R_{2}^{2} \\
x_{C}(y)+R_{1}(y)=x_{C}(0)+R_{1}(0)
\end{array}\right.
$$

where $x_{C}(y)$ is the distance in horizontal direction from the origin to the circumference of the circle with radius $R_{2}$. Using subsitution of parameter $x_{C}(y)$ and the fact that $x_{C}(0)=R_{2}$ the following expression describes $R_{1}(y)$ :

$$
R_{1}(y)=R_{1}(0)+R_{2}-\sqrt{R_{2}^{2}-y^{2}}
$$

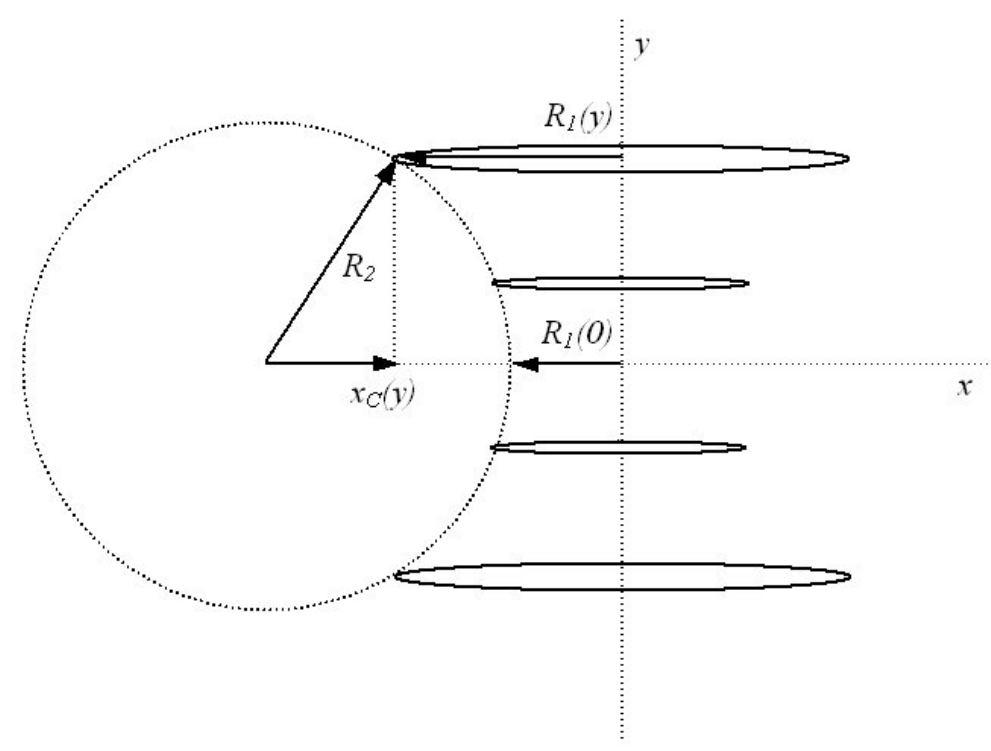

Figure A.2.3.2. Schematic representation of the "Diabolo" structure in terms of the parameters $R_{1}(y)$, $\mathrm{R}_{2}$ and $\mathrm{x}_{\mathrm{C}}(\mathrm{y})$.

If a 3-D margin is generated, only one extra parameter $m$ (margin) has to be added to equation (2) to describe the expanded "Diabolo" structure per $y$-position:

$$
R_{1}(y)=R_{1}(0)+R_{2}-\sqrt{\left(R_{2}-m\right)^{2}-y^{2}}
$$

Use for the values of radii $R_{1}(0)$ and $R_{2}$ e.g.10 and $15 \mathrm{~cm}$ respectively; for the values for the different margins $\mathrm{m} 0.3,0.5$ and $1.0 \mathrm{~cm}$ are suggested. 


\section{A.4.1 Tolerances for the accuracy of dose calculations}

From several studies on the accuracy of photon and electron beam dose calculations (e.g., ICRU [47], Brahme et al. [6], Van Dyk et al. [7], IPEMB [8], SSRPM [10], Venselaar et al. [18]) the following conclusions can be drawn.

a. Very narrow accuracy margins can only be defined for points on the central axis of an open beam with normal incidence, from $d_{\max }$ up to larger depths.

b. Tolerances are defined less strict for points in the build-up region, for oblique incidence, for blocked and wedged fields.

c. For regions with a large dose gradient (build-up region, penumbra), tolerances should be stated in terms of shifts of isodose lines (in $\mathrm{mm}$ ), rather than in dose difference (in \%).

$\mathrm{d}$. The dose at points outside the beam, or below a transmission block is low. In these cases tolerances should rather be expressed relative to the dose value at the central axis of the (open) beam at the same depth.

e. Tolerances may be defined for the maximum deviation and for the average deviation, for example of a larger number of points on a PDD curve.

Tolerances for the accuracy of dose calculations should in principle be expressed as a percentage of the local dose. Such a normalisation is preferred to the use of the dose at $d_{\max }$ or other specific reference depth, e.g. $d_{\text {ref }}=10 \mathrm{~cm}$, of the central beam axis. The local dose eventually determines the success or failure of a radiation treatment and is therefore clinically the most relevant quantity.

\section{A.4.1.1 Tolerances for photon beam dose calculations}

Different tolerances $\delta$ are proposed for the various regions in a photon beam as shown in Fig. A.4.1.1:

a. $\delta_{1}$ : for points on the central beam axis ${ }^{1}$ around and beyond the depth of $d_{\text {max }}$ : the high dose and small dose gradient region.

b. $\delta_{2}$ : for points in the build-up region on the beam axis, in the penumbra, and in regions close to interfaces of inhomogeneities: the high dose and large dose gradient regions. This tolerance criterion may be applied in the region between the phantom surface and 
the depth of the $90 \%$ isodose line or isodose surface, as well as in the penumbra region. As an alternative, the shift of isodose lines (in $\mathrm{mm}$ ) may be used. The dose gradient in these regions is generally larger than $3 \%$ per $\mathrm{mm}$.

c. $\delta_{3}$ : for points beyond $d_{\max }$ within the beam but off the central beam axis: this region is also a high dose and small dose gradient region.

d. $\delta_{4}$ : for points outside the geometrical beam edges, or below shielding blocks, generally beyond $\mathrm{d}_{\text {max }}$ : this region is a low dose and small dose gradient region, for instance below $7 \%$ of the central ray normalization dose.

e. $\delta_{\mathrm{RW} 50}$ : for deviations in the radiological width, defined as the width of a profile measured at the $50 \%$ points (NCS 1996 [48]).

f. $\delta_{50-90}$ : for deviations in the distance between the $50 \%$ and the $90 \%$ point (relative to the value on the beam axis) on the beam profile, the "beam fringe".

Values for the tolerances for the accuracy of photon beam dose calculations are summarized in Table A.4.1.1. The situations for which these accuracy requirements are applicable are:

1. Homogeneous, simple geometry.

For calculation of dose values in homogeneous phantoms for fields without special accessories and without asymmetrical collimator setting the tolerances of the second column under (1) can be applied. These test situations include variation of SSD, rectangular field sizes, oblique incidence.

2. Complex geometry (including a wedge, inhomogeneity, asymmetry).

For dose calculations for complex cases, larger tolerances are acceptable. These situations include beams with wedges, inhomogeneities, irregular fields, missing tissue effects, and asymmetrical collimator settings, but not a combination of these modifications. See under column (2).

3. More complex geometries (combinations of 2).

Applicable for combinations of the irradiation conditions given under (2), such as wedged asymmetrical beams, or inhomogeneities in an irregular field. For the points within the beam edges the tolerance recommended here is different from the tolerance for the

\footnotetext{
${ }^{1}$ The central beam axis corresponds with the collimator rotation axis; in case of an asymmetrically collimated field the central beam axis corresponds with the rayline through the centre of the irradiated field.
} 
points outside the beam. For the large dose gradient region and for the $\mathrm{RW}_{50}$ the criteria are the same as in the former case. See under column (3).

\section{A.4.1.2 Tolerances for electron beam dose calculations}

Tolerances for the accuracy of dose calculations in electron beams should be expressed as a percentage of the local dose as discussed before for photon beams. Different tolerances are proposed for the various regions in an electron beam, shown in Fig. A.4.1.2.

- $\delta_{1}$ : for points on the central beam axis between a depth of $2 \mathrm{~mm}$ and $\mathrm{R}_{95}$, with dose gradients less than $3 \%$ per $\mathrm{mm}$ (i.e. excluding the surface dose points up to a depth of 2 $\mathrm{mm})$ : the high dose and small dose gradient region.

$\delta_{2}$ : for points in regions with a high dose gradient, such as on the central beam axis between $R_{95}$ and $R_{10}$, the penumbra, regions close to interfaces of inhomogeneities: the high dose and large dose gradient regions. The dose gradient is in general larger than $3 \%$ per $\mathrm{mm}$. The tolerance criterion is preferably expressed as a shift of isodose lines (in $\mathrm{mm})$.

- $\delta_{3}$ : for points with a high dose but off the central beam axis and points describing the surface dose: this region is also a high dose and small dose gradient region.

- $\delta_{4}$ : for points outside the geometrical beam edges; this region is a low dose and small dose gradient region, for instance below $7 \%$ of the central beam axis normalization dose.

- $\delta_{\mathrm{RW} 50}$ : for deviations in the radiological width, defined as the width of a profile measured at the $50 \%$ points.

- $\delta_{\mathrm{R} 85}$ and $\delta_{\mathrm{Rp}}$ : for deviations in the therapeutical range and the practical range of the electron beam, respectively.

Values for the tolerances for the accuracy of electron beam dose calculations are summarized in Table A.4.1.2. The situations for which these accuracy requirements are applicable are:

\section{Homogeneous flat phantom, rectangular fields, no inserts}

For calculation of dose values in relatively simple square and rectangular fields without inhomogeneity correction and without field shaping inserts in the field, the values under column 1 can be used. 
2. Irregularly shaped fields, oblique incidence, inhomogeneity and limited SSD variation For dose calculations in other cases, larger tolerances are acceptable. These situations include electron beams which are irregularly shaped with shielding blocks, with oblique incidence, with inhomogeneities in the phantom. The acceptable values are listed under column 2 .

\section{A.4.1.3 Discussion on tolerances}

A fundamental criticism on the set of accuracy requirements presented here is that the suggested tolerances are related to the complexity of the treatment. For instance, why is less accuracy required for treatment of head and neck cancers if wedges and asymmetric beam set-up are used? It is, however, clear that the dose calculation algorithms under these circumstances have a larger uncertainty than for more simple treatment techniques. The proposed values for the criteria presented for individual points and for studies based on e.g. the AAPM [4] or NCS [26] test set, should therefore be considered as a compromise between what is clinically desirable and what can be achieved in practice at this moment. In the future, performance standards for dose calculations of treatment planning systems should converge to a tolerance value of 2 to $3 \%$ for all situations. 

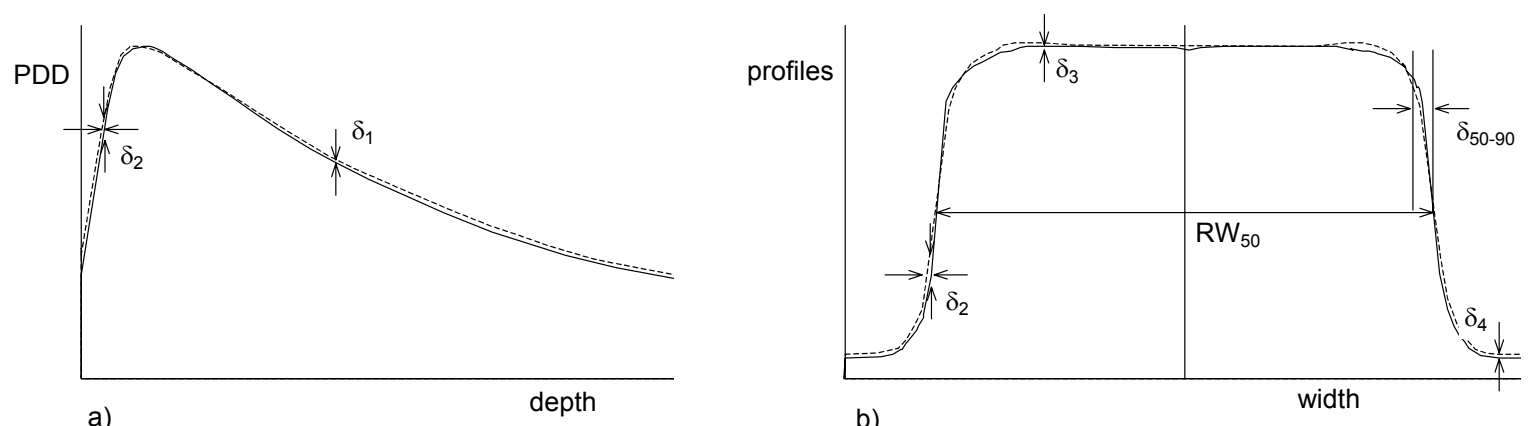

Fig. A.4.1.1 Different tolerances are proposed for the various regions in a photon beam; (a) depthdose curve: (b) beam profile.
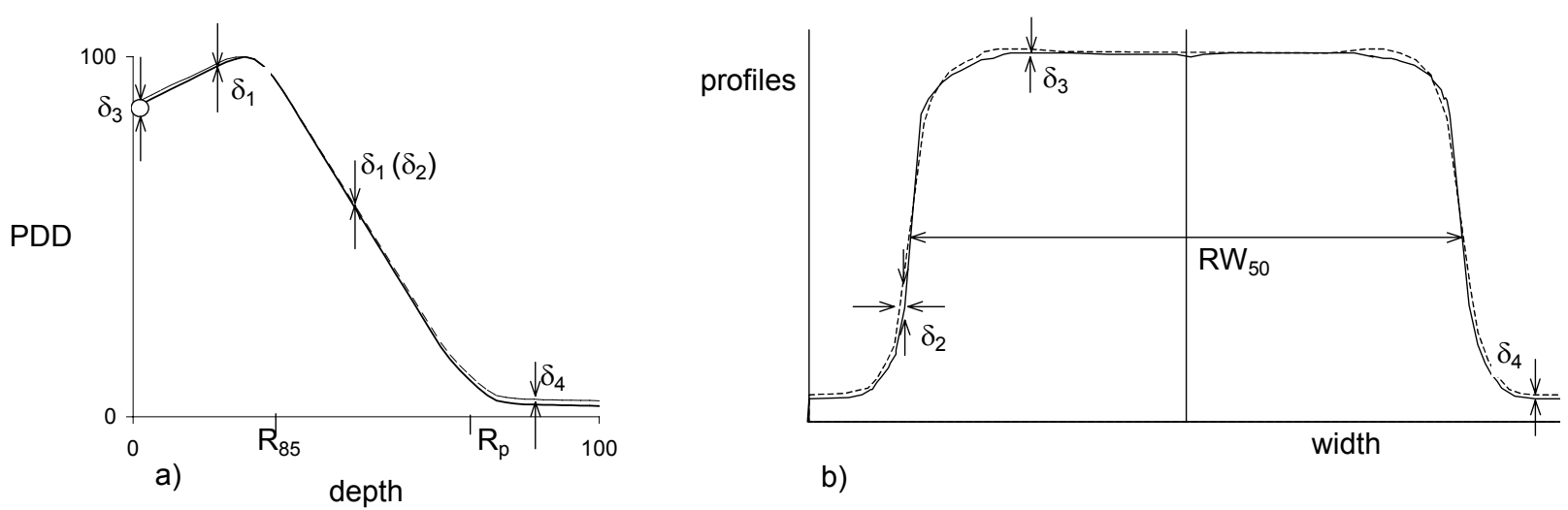

b)

Fig. A.4.1.2 Different tolerances are proposed for the various regions in an electron beam; (a) a depthdose curve (b) a beam profile. 
Table A.4.1.1. Tolerances for the accuracy of photon beam dose calculations.

\begin{tabular}{|c|c|c|c|}
\hline Region & $\begin{array}{c}(1) \\
\text { homogeneous, } \\
\text { simple geometry }\end{array}$ & $\begin{array}{c}(2) \\
\text { complexity } \\
\text { (wedge, inhom., } \\
\text { asymm., etc) }\end{array}$ & $\begin{array}{l}(3) \\
\text { more complex, i.e } \\
\text { combinations } \\
\text { of (2) }\end{array}$ \\
\hline $\begin{array}{c}\delta_{1} \\
\text { (central beam axis data) } \\
\text { high dose, } \\
\text { small dose gradient }\end{array}$ & $2 \%$ & $3 \%$ & $4 \%$ \\
\hline $\begin{array}{c}\delta_{2}{ }^{1} \\
\text { (build-up region of central beam axis, } \\
\text { penumbra region of the profiles) } \\
\text { high dose, } \\
\text { large dose gradient }\end{array}$ & $\begin{array}{c}2 \mathrm{~mm} \\
\text { or } \\
10 \%\end{array}$ & $\begin{array}{l}3 \mathrm{~mm} \\
\text { or } \\
15 \%\end{array}$ & $\begin{array}{l}3 \mathrm{~mm} \\
\text { or } \\
15 \%\end{array}$ \\
\hline $\begin{array}{c}\delta_{3} \\
\text { (outside central beam axis region) } \\
\text { high dose, } \\
\text { small dose gradient }\end{array}$ & $3 \%$ & $3 \%$ & $4 \%$ \\
\hline $\begin{array}{c}\delta_{4} \\
\text { (outside beam edges) } \\
\text { low dose, } \\
\text { small dose gradient }\end{array}$ & $\begin{array}{c}3 \%{ }^{2} \\
(30 \%)\end{array}$ & $\begin{array}{c}4 \%{ }^{2} \\
(40 \%)\end{array}$ & $\begin{array}{l}5 \%{ }^{2} \\
(50 \%)\end{array}$ \\
\hline $\begin{array}{c}\delta_{\mathrm{RW} 50} \\
\text { (radiological width) }\end{array}$ & $\begin{array}{l}2 \mathrm{~mm} \\
\text { or } \\
1 \%\end{array}$ & $\begin{array}{l}2 \mathrm{~mm} \\
\text { or } \\
1 \%\end{array}$ & $\begin{array}{l}2 \mathrm{~mm} \\
\text { or } \\
1 \%\end{array}$ \\
\hline $\begin{array}{c}\delta_{50-90} \\
\text { (beam fringe) }\end{array}$ & $2 \mathrm{~mm}$ & $3 \mathrm{~mm}$ & $3 \mathrm{~mm}$ \\
\hline
\end{tabular}

${ }^{1}$ These data are preferably expressed in $\mathrm{mm}$ shift. A shift of $1 \mathrm{~mm}$ corresponding to a dose variation of $5 \%$ is assumed to be a realistic value in the high dose - large dose gradient region.

${ }^{2}$ This figure is normalized to the dose at a point at the same depth on the central beam axis; the percentage in brackets refers to the local dose. 
Table A.4.1.2 Summary of the tolerances for electron beam dose calculations

\begin{tabular}{|c|c|c|}
\hline Tolerance & $\begin{array}{l}\text { (1) } \\
\text { homogeneous, } \\
\text { no inserts }\end{array}$ & $\begin{array}{l}\text { (2) } \\
\text { irregular shape, } \\
\text { oblique fields, inserts }\end{array}$ \\
\hline $\begin{array}{c}\delta_{1} \\
\text { (central beam axis data) } \\
\text { high dose, small dose gradient }\end{array}$ & $2 \%$ & $3 \%$ \\
\hline $\begin{array}{c}\delta_{2} \\
\text { (central axis points in low energy beams, } \\
\text { penumbra region of the profiles) } \\
\text { high dose, large dose gradient }\end{array}$ & $\begin{array}{l}2 \mathrm{~mm} \\
\text { or } \\
2 \%\end{array}$ & $\begin{array}{l}3 \mathrm{~mm} \\
\text { or } \\
10 \%\end{array}$ \\
\hline $\begin{array}{l}\delta_{3} \\
\text { (outside central beam axis region, } \\
\text { points in the build-up region) } \\
\text { high dose, small dose gradient }\end{array}$ & $3 \%$ & $4 \%$ \\
\hline $\begin{array}{c}\delta_{4}{ }^{1} \\
\text { (outside beam edges) } \\
\text { low dose, small dose gradient }\end{array}$ & $2 \%$ & $4 \%$ \\
\hline $\begin{array}{c}\delta_{\mathrm{RW} 50} \\
\text { (radiological width) }\end{array}$ & $4 \mathrm{~mm}$ & $4 \mathrm{~mm}$ \\
\hline $\begin{array}{c}\delta_{R p} \text { and } \delta_{R 85} \\
\text { (practical and therapeutic range) }\end{array}$ & $2 \mathrm{~mm}$ & $3 \mathrm{~mm}$ \\
\hline
\end{tabular}

${ }^{1}$ This percentage results from normalization to the dose at a point at the same depth on the central beam axis. 


\section{A.4.2 Test configurations}

\section{A.4.2.1 The basic beam dataset; measurement and definition}

Methods for the determination of basic beam data should be well described and applied to each beam quality and type of treatment machine. Preferably the same equipment (ionization chamber, electrometer, semiconductor detector, water phantom) should be used for the measurements. Measurement of data of the same beam performed by more than one investigator, or on different days, or in different circumstances (for example after repair of the machine) should be checked for consistency by using redundancy measurements. For this purpose, re-measurement of at least one reference percentage depth dose curve and one beam profile is recommended; measurement of a number of reference square field output factors is recommended in the case this type of data has to be obtained. The influence of periodic maintenance, of warm-up periods after switching-on, and interruptions during the measurement sessions of the reference dataset should be excluded.

In a treatment planning program, basic beam data are entered for the purpose of modeling the beam. These basic data are generally derived from measurements in a homogeneous medium, e.g., by using suitable detectors in a large water phantom. These data should encompass the range of field sizes and depths used in clinical situations for an interpolation based TPS. The phantom should be large enough to have full scatter conditions, e.g. extending at least $5 \mathrm{~cm}$ beyond the maximum field borders and maximum depth of measurement. The data should be sufficient to build the model in systems with a physical beam modeling type of algorithm. We refer to these data as the basic beam dataset.

The basic beam dataset for photon beams is usually composed of PDD (or TPR) data for a series of square fields with sides with fixed intervals (e.g. 4, 6, 8, 10, .. $40 \mathrm{~cm}$ ), or with gradually increasing intervals (e.g. $3,4,5,6,7,8,10, \ldots, 20,25,30,35,40 \mathrm{~cm}$ ). Depths in the phantom should have a small interval in the region around $d_{\max }$ (e.g. $0.5 \mathrm{~cm}$ from $d=0$ up to $d=5 \mathrm{~cm}$ ), but may have intervals of $2-3 \mathrm{~cm}$ at larger depths, up to $30 \mathrm{~cm}$. Output factors should be measured for the same field sizes. Due to the collimator exchange effect of a megavoltage treatment unit, it is often necessary to determine output factors for independent $\mathrm{X}$ - and Y-settings. Profiles should be measured for at least 4 field sizes, e.g., $5 \mathrm{~cm} \times 5 \mathrm{~cm}, 10$ $\mathrm{cm} \times 10 \mathrm{~cm}, 20 \mathrm{~cm} \times 20 \mathrm{~cm}$ and $40 \mathrm{~cm} \times 40 \mathrm{~cm}$, at at least 4 depths, e.g., $\mathrm{d}_{\max }, 5,10$ and 20 $\mathrm{cm}$. Profiles should be measured to at least $5 \mathrm{~cm}$ outside the geometrical beam edge. For 
wedged beams and treatment units with asymmetrical collimators, the same measurements should be repeated in addition to the open and symmetrical beam settings.

The basic beam dataset for electron beams is usually composed of PDD data for a series of square fields with sides with fixed intervals (e.g., 4, 6, 8, $10 \mathrm{~cm}, \ldots$ to maximum field size), or according to the standard inserts delivered with the applicator system of the linear accelerator. Depths in the phantom generally should have a small interval (e.g., $0.5 \mathrm{~cm}$ from $d=0$ up to the practical range of the electrons, $R_{p}$ ), and must include at least a few points in the photon background beyond $R_{p}$. Output factors should be measured for the same field sizes. Profiles should be measured for at least 4 representative field sizes and at least at 4 different depths. Profiles should be measured to at least $5 \mathrm{~cm}$ outside the geometrical beam edge.

The treatment planning software may require a specified set of data. Care should be taken to follow the definitions in the program or user's manuals of the quantities to be measured. For instance, the depth of measurement of output factors or the use of a fixed SSD or isocentric detector set-up is often specified for this purpose.

The basic beam dataset covers the range of fields and depths used clinically and the data must be checked for internal consistency. Reference measurements should be defined. If data are obtained at different days, the reference measurements should be repeated. Reproducibility of relative depth-dose data should then be better than $1 \%$ of the local PDD value. Some smoothing may be applied, if required.

\section{A.4.2.2 The test dataset; definition and description}

The TPS should be able to reproduce the basic beam data -after the beam modeling process- with high accuracy. Additional data are needed to check the performance of the algorithm in clinically relevant circumstances. We define a second dataset, the test dataset to be used for this purpose. The test dataset consists of dose values, measured at specified points in a number of representative beam geometries, for a given number of monitor (time) units of the treatment machine.

The data points for photon beams should be measured at depths between 0 and $30 \mathrm{~cm}$, in rectangular fields with $X$ - and $Y$-setting between 4 and $40 \mathrm{~cm}$, with SSD varying between 75 and $150 \mathrm{~cm}$. Depending on which treatment set-ups are applied clinically, additional data 
should be available for beams with beam modifiers (tray, wedge), inhomogeneities (with different sizes and densities) and with deviating contours. In order to avoid the problems of many algorithms with the calculation of dose near interfaces, points close to the surface of the phantom or close to inhomogeneities are generally not part of the test dataset. If special irradiation techniques are applied in a department, such as total body irradiation, or radiosurgery, a dedicated test procedure should be developed.

A test dataset for photon beams has been proposed by AAPM Task Group 23 [4]. A set of basic beam data measurements was first performed using a $4 \mathrm{MV}$ and a $18 \mathrm{MV}$ photon beam. Then, the test dataset consists of dose values at a number of points in square and rectangular beams, on-axis and off-axis; a beam with a wedge, central block, irregular block, inhomogeneities, and oblique incidence of the beam. The set proposed by the TG 23 has a number of limitations. The treatment units used for the set are outdated at this moment and not very common in European radiotherapy departments. New technical developments, such as asymmetric collimators and multi-leaf collimators, are not available in the set and cannot be added from these old machines. The set is, therefore, static and has a limited applicability in the current clinical practice. A new set of data was therefore measured in order to overcome these disadvantages. Based on the AAPM TG 23 proposal, new tests were defined including missing tissue, asymmetrical fields and asymmetrical wedged fields. These data were measured at the radiotherapy department of the University Medical Center in Utrecht, with photon beams of state-of-the-art linear accelerators (SL 15 and SL 25, EOS, Crawley UK). This type of linear accelerator is used in many hospitals in The Netherlands. We refer to this set as the NCS set, which has been described more extensively elsewhere (Venselaar and Welleweerd [26]).

When collecting the data of the test set, similar care must be taken as during the measurement of the basic beam data set to have optimal internal consistency. The reference measurements (see above) must be repeated with the same criterion for relative depth-dose data $(<1 \%$ of the local dose).

A description of the test situations for photon beams is given in Section A.4.2.3 The tests are illustrated in the corresponding Figures A.4.2.1 and A.4.2.2. A description of the test geometries for electron beams is given in Section A.4.2.4. 
- The proposed set is a modification of the set originally proposed for this purpose by AAPM TG 23 [4]. The modifications refer to modern treatment equipment and include the use of a motorized $60^{\circ}$ wedge, asymmetric collimators and multi-leaf collimators as well as an additional test for missing tissue.

- Some block specifications are left to the choice of the user to accommodate to the materials used clinically.

- Unless specified otherwise, points of measurement are at 1, 3, 5, 10, 15, 20, 25, and 30 $\mathrm{cm}$ depth. Distances from the central axis of the beam as indicated in the figures. One of the depths may be adapted to the depth of $d_{\max }$, depending on the photon beam quality under investigation.

- For the numbering of the different tests the original AAPM TG 23 proposal has been used, to which other tests were added.

\section{A.4.2.3 Description of the tests for photon beams}

1.a. Open field, $5 \mathrm{~cm} \times 5 \mathrm{~cm}, \mathrm{SSD}=100 \mathrm{~cm}$, in a transverse plane through the central axis of the beam.

1.b. Open field, $10 \mathrm{~cm} \times 10 \mathrm{~cm}, \mathrm{SSD}=100 \mathrm{~cm}$, in a transverse plane through the central axis of the beam.

1.c. Open field, $25 \mathrm{~cm} \times 25 \mathrm{~cm}, \mathrm{SSD}=100 \mathrm{~cm}$, in a transverse plane through the central axis of the beam.

2.a. Open field, $5 \mathrm{~cm} \times 25 \mathrm{~cm}, \mathrm{SSD}=100 \mathrm{~cm}$, in a transverse plane through the central axis of the beam.

2.b. Open field, $25 \mathrm{~cm} \times 5 \mathrm{~cm}, \mathrm{SSD}=100 \mathrm{~cm}$, in a transverse plane through the central axis of the beam.

3. SSD variation, open field, $10 \mathrm{~cm} \times 10 \mathrm{~cm}$ (at SAD), SSD $=85 \mathrm{~cm}$, in a transverse plane through the central axis of the beam.

4. $60^{\circ}$ wedged field, $9 \mathrm{~cm} \times 9 \mathrm{~cm}, \mathrm{SSD}=100 \mathrm{~cm}$, in a transverse plane through the central axis of the beam. 
5. Centrally blocked field, $16 \mathrm{~cm} \times 16 \mathrm{~cm}, \mathrm{SSD}=100 \mathrm{~cm}$, in a transverse plane through the central axis of the beam. Block of (for example) $7 \mathrm{~cm} \times 2 \mathrm{~cm}$, positioned on the central beam axis on the blocking tray; thickness and material -defining the block transmission- according to the material available in the radiotherapy department.

6. Off-plane, open field, $10 \mathrm{~cm} \times 10 \mathrm{~cm}, \mathrm{SSD}=100 \mathrm{~cm}$, in a plane $4 \mathrm{~cm}$ off axis.

7. Irregular blocked field, L-shaped, $16 \mathrm{~cm} \times 16 \mathrm{~cm}, \mathrm{SSD}=100 \mathrm{~cm}$, in a transverse plane through the central axis of the beam. A large block of $12 \mathrm{~cm} \times 12 \mathrm{~cm}$, positioned on the blocking tray; thickness and material (defining the block transmission) according to the material available in the radiotherapy department.

8.a. Inhomogeneity, open field, $6 \mathrm{~cm} \times 6 \mathrm{~cm}, \mathrm{SSD}=100 \mathrm{~cm}$, in a transverse plane through the central axis of the beam. Lung inhomogeneity, diameter $6.0 \mathrm{~cm}$, density for example 0.3 g. $\mathrm{cm}^{-3}$, depth of centre of the inhomogeneity $8 \mathrm{~cm}$.

8.b. Inhomogeneity, open field, $16 \mathrm{~cm} \times 16 \mathrm{~cm}$, SSD $=100 \mathrm{~cm}$, in a transverse plane through the central axis of the beam. Lung inhomogeneity, diameter $6.0 \mathrm{~cm}$, density for example 0.3 g.cm ${ }^{-3}$, depth of centre $8 \mathrm{~cm}$.

8.c. Inhomogeneity, open field, $16 \mathrm{~cm} \times 16 \mathrm{~cm}$, SSD $=100 \mathrm{~cm}$, in a transverse plane through the central axis of the beam. Bone inhomogeneity, diameter $2.0 \mathrm{~cm}$, density for example 1.8 g.cm ${ }^{-3}$, depth of centre $6 \mathrm{~cm}$.

9. Oblique incidence, open field, $10 \mathrm{~cm} \times 10 \mathrm{~cm}, \mathrm{SSD}=100 \mathrm{~cm}$, in a transverse plane through the central axis of the beam. Gantry position at $45^{\circ}$.

10.a. Missing tissue, open field, $10 \mathrm{~cm} \times 10 \mathrm{~cm}$, SSD $=100 \mathrm{~cm}$, in a transverse plane through the central axis of the beam. Half of the beam outside the phantom. In practice, a gantry position at $90^{\circ}$ can be used for the measurement with the central beam axis leveled with the water surface.

10.b. Missing tissue, open field, $20 \mathrm{~cm} \times 20 \mathrm{~cm}, \mathrm{SSD}=100 \mathrm{~cm}$, in a transverse plane through the central axis of the beam. Again, half of the beam outside the phantom. 
11. Half and quarter fields, open field, $15 \mathrm{~cm} \times 15 \mathrm{~cm}$, SSD $=100 \mathrm{~cm}$, with asymmetric setting of the collimator in $\mathrm{X}$ - and $\mathrm{Y}$-direction.

12. Half and quarter fields, wedged field, $15 \mathrm{~cm} \times 15 \mathrm{~cm}$, SSD $=100 \mathrm{~cm}$, with asymmetric setting of the collimator in $\mathrm{X}$ - and $\mathrm{Y}$-direction.

13. MLC tests for open fields. To limit the number of measurements, only depths equal to $\mathrm{d}_{\max }, 5$ and $10 \mathrm{~cm}$ are considered. The outline of the fields is schematically shown in Fig. A.4.2.2.a-c, and the corresponding settings of the individual collimator leafs and jaws are given in Table A.4.2.1.

14. MLC tests for open fields with large asymmetrical setting of the beam. The field outlines are the same as described in test 13.a, but with an off-set position of $X$ and $Y$ to $(10,0)$ and $(10,10) \mathrm{cm}$, respectively. Only depths $d_{\max }, 5$ and $10 \mathrm{~cm}$ are considered. This test of asymmetrical settings is limited to the examples 1,2 and 12 of Table A.4.2.1.

\section{A.4.2.4 Description of the tests for electron beams}

The following test geometries, see Fig. A.4.2.3, are proposed for electron beams (note: electron beam tests are not included in the NCS test package):

1. Square fields, a) small field of $5 \mathrm{~cm} \times 5 \mathrm{~cm}$; and b) reference field of $10 \mathrm{~cm} \times 10 \mathrm{~cm}$. Points include the C-ax PDD and off-axis points at 3 depths; one at $\mathrm{d}_{\max }$ and 2 depths in the range between $d_{\max }$ and $d_{50 \%}$.

2. Rectangular fields, a) field of $5 \mathrm{~cm} \times 20 \mathrm{~cm}$; and b) field of $20 \mathrm{~cm} \times 5 \mathrm{~cm}$. Points include the $\mathrm{C}$-ax PDD and off-axis points at 3 depths; one at $\mathrm{d}_{\max }$ and 2 depths in the range between $d_{\max }$ and $d_{50 \%}$.

3. Irregular fields, a) field of $5 \mathrm{~cm} \times 10 \mathrm{~cm}$, blocked to a $5 \mathrm{~cm} \times 5 \mathrm{~cm}$ field (asymmetrical field, i.e. with the collimator rotation axis at the edge of the field); and b) field of $10 \mathrm{~cm} \times 20 \mathrm{~cm}$, blocked to $10 \mathrm{~cm} \times 10 \mathrm{~cm}$ field (asymmetrical field, i.e. with the collimator rotation axis at the edge of the field). 
4. Oblique incidence, field $10 \mathrm{~cm} \times 10 \mathrm{~cm}$, with gantry angle of $45^{\circ}$. Points representing the C-ax PDD and 3 profile depths.

5. Extended SSD, field $10 \mathrm{~cm} \times 10 \mathrm{~cm}$ at $S S D=110 \mathrm{~cm}$. Points include the $\mathrm{C}$-ax PDD and off-axis points at 3 depths; one at $d_{\max }$ and 2 depths in the range between $d_{\max }$ and $d_{50 \%}$.

6. Inhomogeneity correction, field $10 \mathrm{~cm} \times 10 \mathrm{~cm}$, a) slab of air (2 cm thick); and b) slab of high density material (bone equivalent, $2 \mathrm{~cm}$ thick). Points representing the C-ax PDD at central beam axis and off-axis points at 2 depths.

7. Inhomogeneity correction, field $10 \mathrm{~cm} \times 10 \mathrm{~cm}$, a) cylinder of air ( $2 \mathrm{~cm}$ diameter); and b) cylinder of high density material (bone equivalent, $2 \mathrm{~cm}$ diameter). Points representing the $\mathrm{C}$ ax PDD at central beam axis and off-axis points at 2 depths.

8. Abutting fields, a) electrons to electrons, two fields $10 \mathrm{~cm} \times 10 \mathrm{~cm}$ of equal electron beam quality, of which the central beam axes are at $10 \mathrm{~cm}$ distance; and b) photons and electrons, in the same set-up. Points representing 3 profile depths; one at $d_{\max } ; 2$ additional profile depths between $d_{\max }$ and $d_{50 \%}$.

Note: to limit the workload, the tests can be performed for a selection of electron beam qualities covering the available range of beam qualities. 


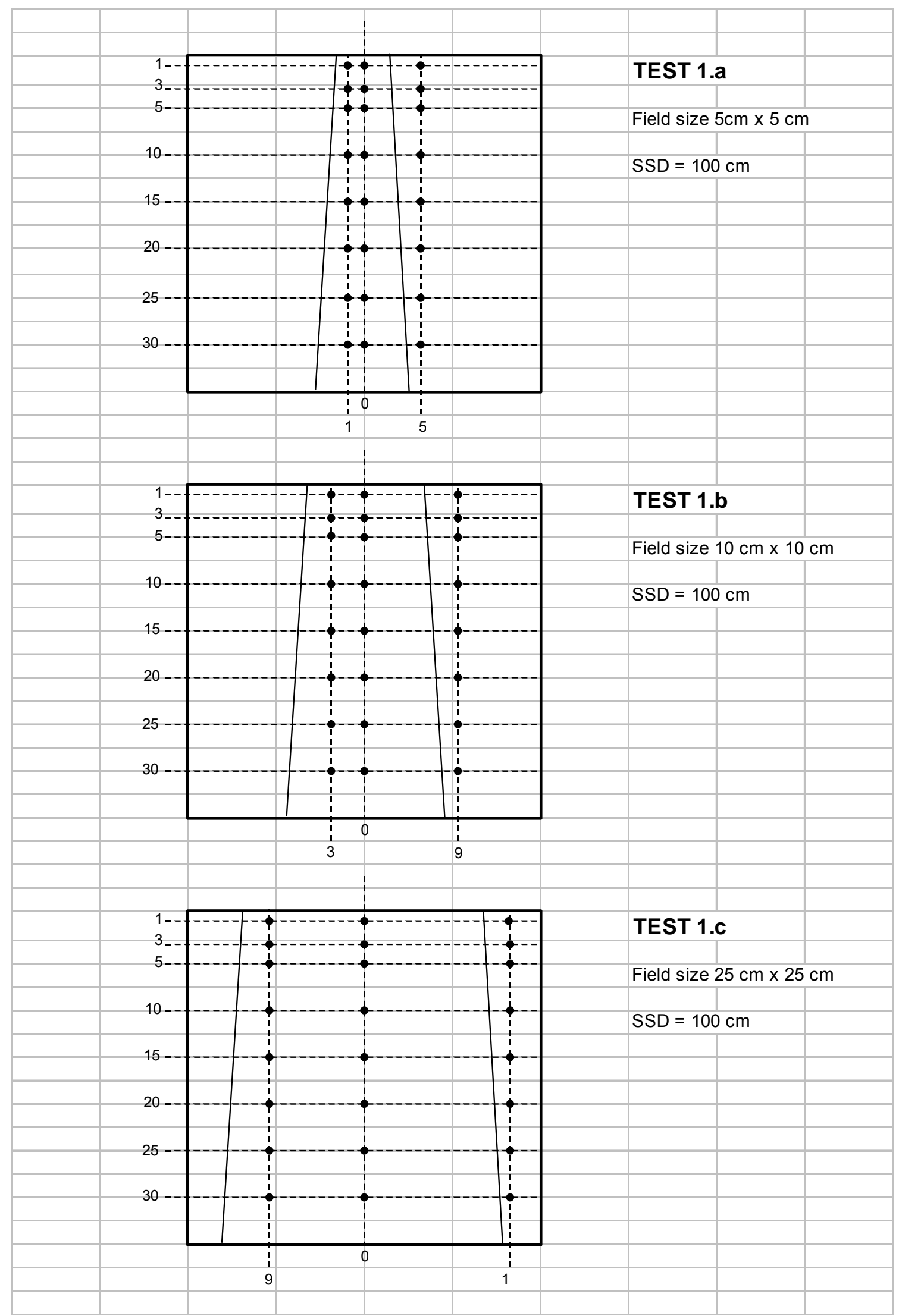

Fig. A.4.2.1 


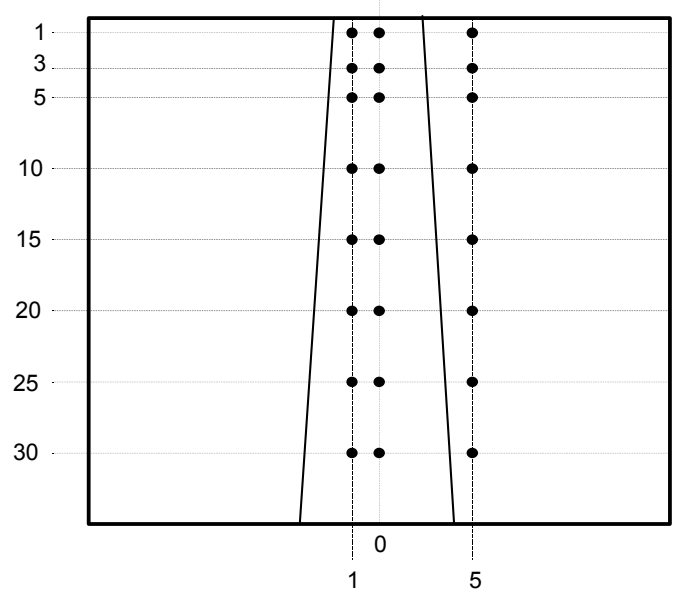

TEST 2.a

Field size $5 \mathrm{~cm} \times 25 \mathrm{~cm}$ $S S D=100 \mathrm{~cm}$

\section{TEST 2.b}

Field size $25 \mathrm{~cm} \times 5 \mathrm{~cm}$ $S S D=100 \mathrm{~cm}$

\section{TEST 3}

Field size $10 \mathrm{~cm} \times 10 \mathrm{~cm}$ (at SAD)

SSD variation

$\mathrm{SSD}=85 \mathrm{~cm}$

Fig. A.4.2.1 (continued) 

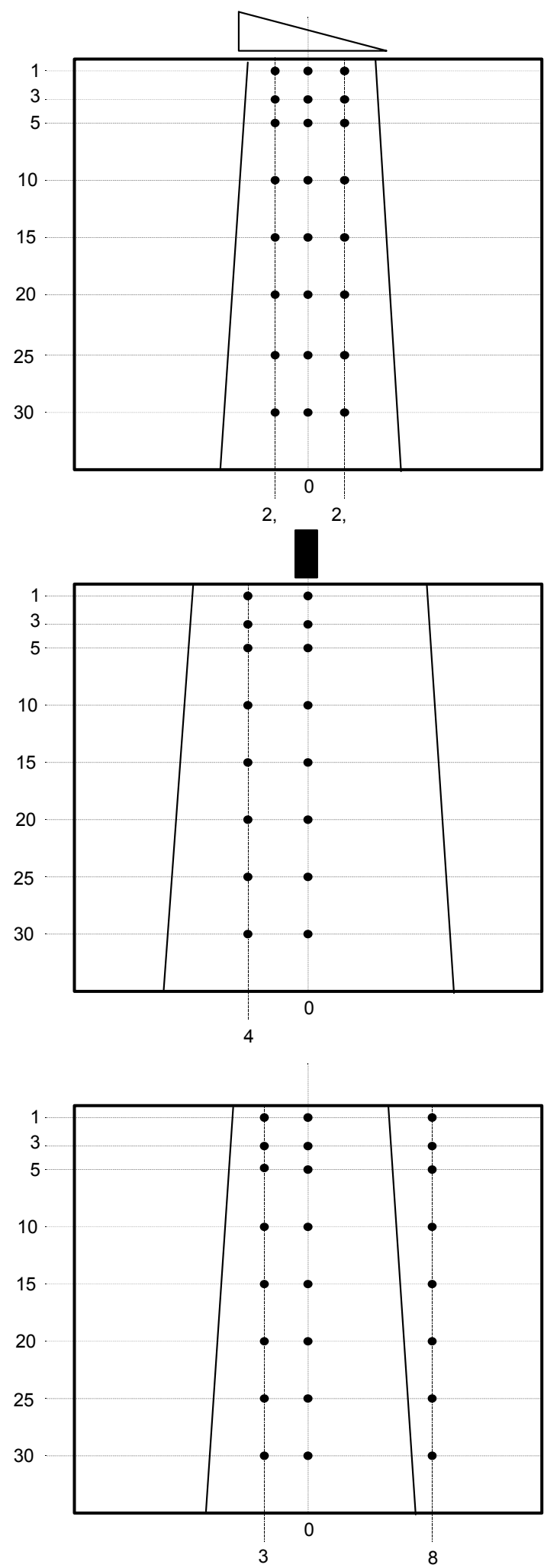

\section{TEST 4}

Field size $9 \mathrm{~cm} \times 9 \mathrm{~cm}$

$\mathrm{SSD}=100 \mathrm{~cm}$

60 degree wedge

\section{TEST 5}

Field size $16 \mathrm{~cm} \times 16 \mathrm{~cm}$

$S S D=100 \mathrm{~cm}$

Central block: $7 \mathrm{~cm} \mathrm{x} 2 \mathrm{~cm}$

\section{TEST 6}

Field size $10 \mathrm{~cm} \times 10 \mathrm{~cm}$

$\mathrm{SSD}=100 \mathrm{~cm}$

Plane $4 \mathrm{~cm}$ off axis

Fig. A.4.2.1 (continued) 


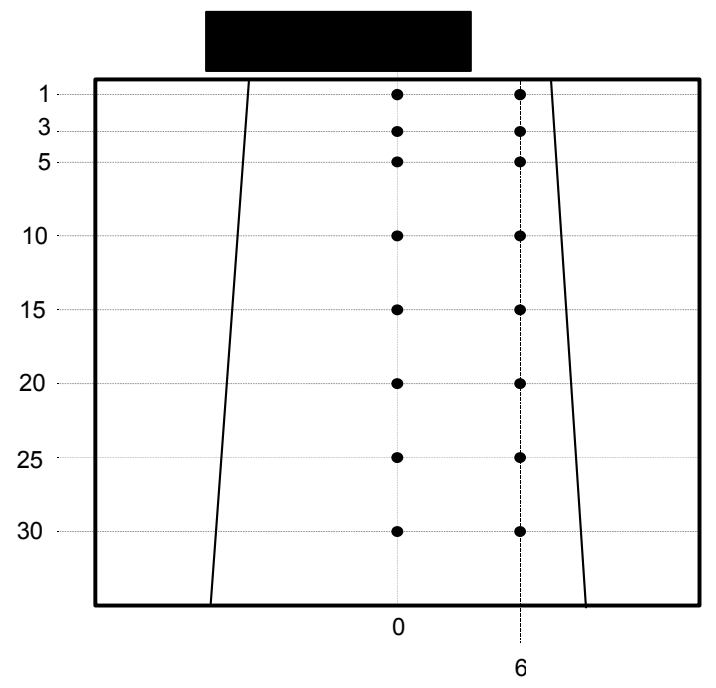

\section{TEST 7}

Field size $16 \mathrm{~cm} \times 16 \mathrm{~cm}$

$S S D=100 \mathrm{~cm}$

Irregular block: $12 \mathrm{~cm} \times 12 \mathrm{~cm}$

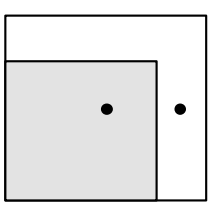

\section{TEST 8.a}

Field size $6 \mathrm{~cm} \times 6 \mathrm{~cm}$

$S S D=100 \mathrm{~cm}$

Inhomogeneity: lung

Diameter: $6 \mathrm{~cm}$

Density: $0.3 \mathrm{~g} \cdot \mathrm{cm}^{-3}$

Depth of centre: $8 \mathrm{~cm}$

\section{TEST 8.b}

Field size $16 \mathrm{~cm} \times 16 \mathrm{~cm}$

$S S D=100 \mathrm{~cm}$

Inhomogeneity: lung

Diameter: $6 \mathrm{~cm}$

Density: $0.3 \mathrm{~g} . \mathrm{cm}^{-3}$

Depth of centre: $8 \mathrm{~cm}$

Fig. A.4.2.1 (continued) 


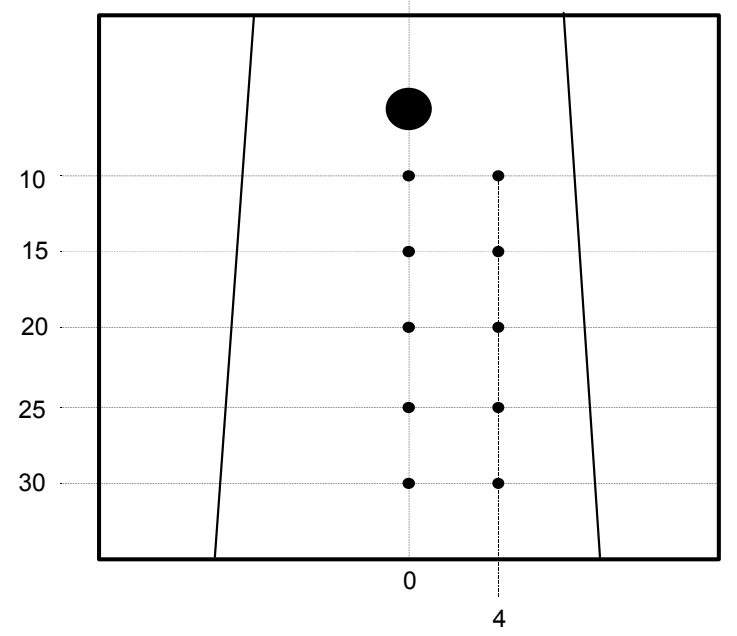

TEST 8.c

Field size $16 \mathrm{~cm} \times 16 \mathrm{~cm}$

$\mathrm{SSD}=100 \mathrm{~cm}$

Inhomogeneity: bone

Diameter: $2 \mathrm{~cm}$

Density: $1.8 \mathrm{~g} \cdot \mathrm{cm}^{-3}$

Depth of centre: $6 \mathrm{~cm}$

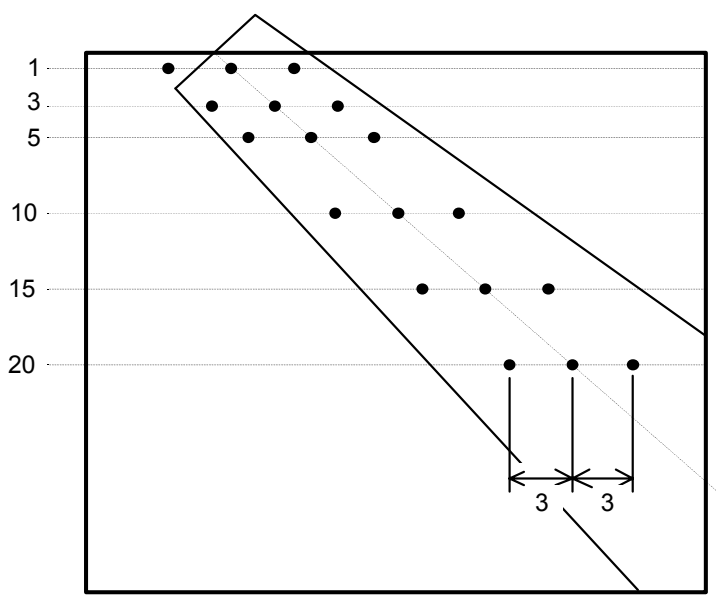

\section{TEST 9}

Field size $10 \mathrm{~cm} \times 10 \mathrm{~cm}$

$S S D=100 \mathrm{~cm}$

Oblique incidence

(gantry $45^{\circ}$ )

Fig. A.4.2.1 (continued) 


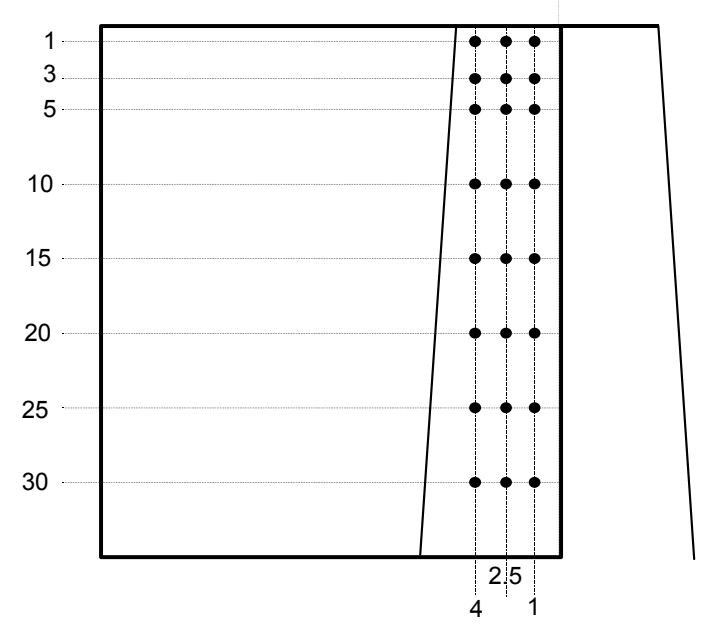

TEST 10.a

Field size $10 \mathrm{~cm} \times 10 \mathrm{~cm}$

$\mathrm{SSD}=100 \mathrm{~cm}$

Half beam:

"missing tissue"

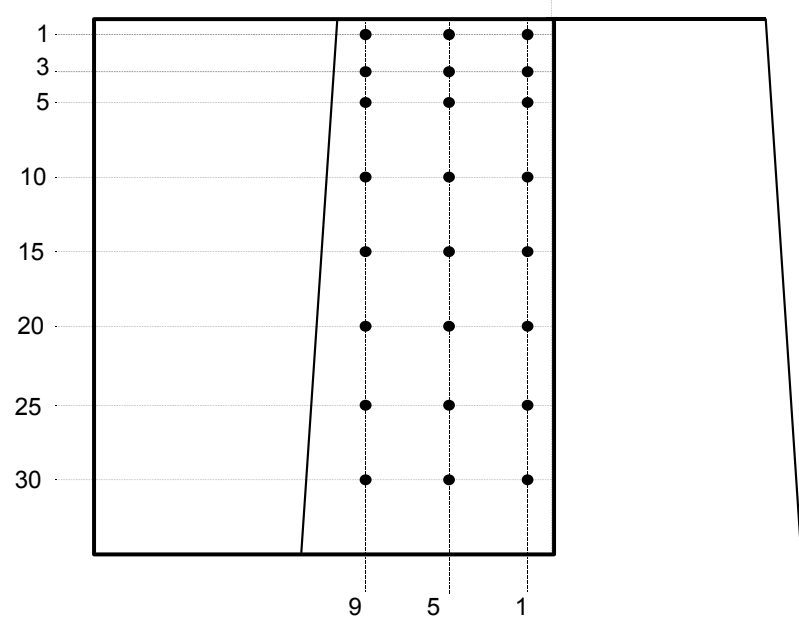

\section{TEST 10.b}

Field size $20 \mathrm{~cm} \times 20 \mathrm{~cm}$

$S S D=100 \mathrm{~cm}$

Half beam:

"missing tissue"

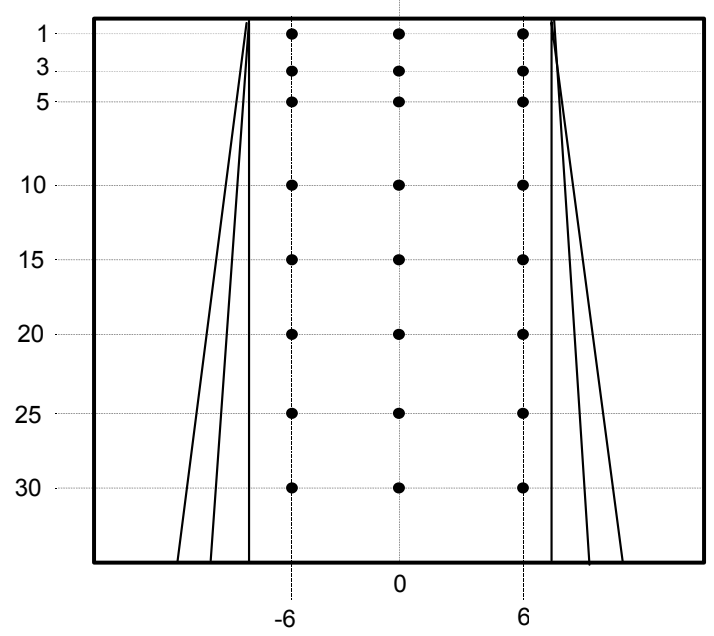

TEST 11

Asymmetrical open field TEST 12

Asymmetrical wedged field

Field size $15 \mathrm{~cm} \times 15 \mathrm{~cm}$ $S S D=100 \mathrm{~cm}$

Isocentre positions:

Fig. A.4.2.1 (continued) 
a)

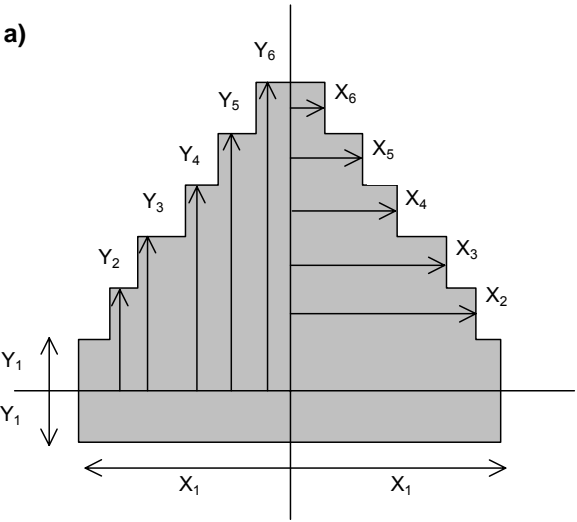

b)

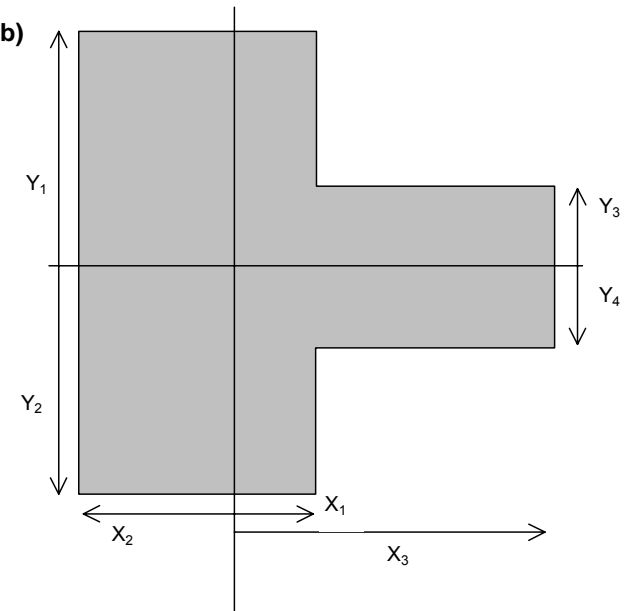

c)

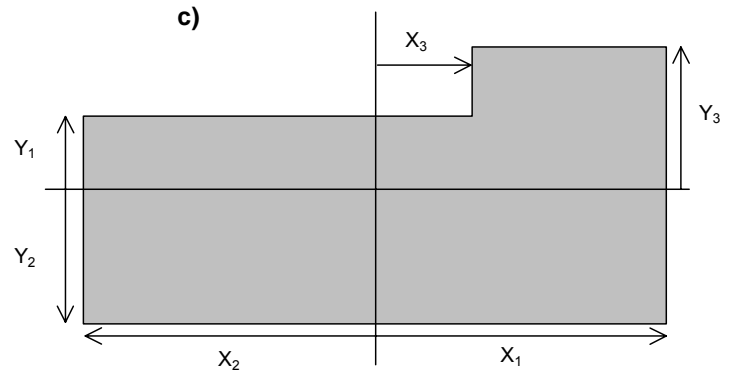

Fig. A.4.2.2 Irregular fields, defined by a multi-leaf collimator. Three basic forms (a-c) are shown of which the field size dimensions are determined by the parameters $x_{i}$ and $y_{i}$. Values for these parameters are given in Table A.4.2.1 for MLCs with a leaf width of $1.0 \mathrm{~cm}$. It is assumed here that the leaves can move in the $\mathrm{x}$-direction. 
Table A.4.2.1 Leaf settings for the fields in Fig. A.4.2.2.a-c for test 13, for an MLC with a leaf width of $1.0 \mathrm{~cm}$ at SAD. A subset is of three fields is defined for use in test 14 in which the field centre is shifted to the asymmetrical position $(10,0)$ and $(10,10)$ (bold in the table).

\begin{tabular}{|c|c|c|c|c|c|c|c|c|c|c|c|c|c|}
\hline$\#$ & $\begin{array}{l}\text { re: Fig. } \\
\text { A.4.2.2 }\end{array}$ & \multicolumn{12}{|c|}{ setting (in cm) } \\
\hline & & $x_{1}$ & $\mathrm{X}_{2}$ & $x_{3}$ & $\mathbf{x}_{4}$ & $x_{5}$ & $x_{6}$ & $\mathrm{y}_{1}$ & $y_{2}$ & $y_{3}$ & $\mathrm{y}_{4}$ & $\mathrm{y}_{5}$ & $\mathrm{y}_{6}$ \\
\hline 1 & A & 6.0 & 5.0 & 4.0 & 3.0 & 2.0 & 1.0 & 2.0 & 3.0 & 4.0 & 5.0 & 6.0 & 7.0 \\
\hline & & & & & & & & & & & & & \\
\hline 2 & B & 2.0 & 4.0 & 8.0 & - & - & - & 6.0 & 6.0 & 2.0 & 2.0 & - & - \\
\hline 3 & $\mathrm{~B}$ & 2.0 & 4.0 & 8.0 & - & - & - & 8.0 & 8.0 & 2.0 & 2.0 & - & - \\
\hline 4 & $B$ & 2.0 & 4.0 & 8.0 & - & - & - & 10.0 & 10.0 & 2.0 & 2.0 & - & - \\
\hline 5 & $\mathrm{~B}$ & 2.0 & 4.0 & 8.0 & - & - & - & 12.0 & 12.0 & 2.0 & 2.0 & - & - \\
\hline 6 & $B$ & 2.0 & 6.0 & 6.0 & - & - & - & 6.0 & 6.0 & 2.0 & 2.0 & - & - \\
\hline 7 & $B$ & 2.0 & 6.0 & 6.0 & - & - & - & 12.0 & 12.0 & 2.0 & 2.0 & - & - \\
\hline 8 & $B$ & 2.0 & 4.0 & 6.0 & - & - & - & 6.0 & 6.0 & 3.0 & 3.0 & - & - \\
\hline 9 & $B$ & 2.0 & 4.0 & 6.0 & - & - & - & 8.0 & 4.0 & 5.0 & 1.0 & - & - \\
\hline 10 & $b$ & 2.0 & 4.0 & 6.0 & - & - & - & 10.0 & 2.0 & 7.0 & -1.0 & - & - \\
\hline 11 & C & 6.0 & 6.0 & 2.0 & - & - & - & 2.0 & 4.0 & 4.0 & - & - & - \\
\hline 12 & C & 6.0 & 6.0 & 0.0 & - & - & - & 2.0 & 4.0 & 4.0 & - & - & - \\
\hline 13 & C & 6.0 & 6.0 & -2.0 & - & - & - & 2.0 & 4.0 & 4.0 & - & - & - \\
\hline
\end{tabular}



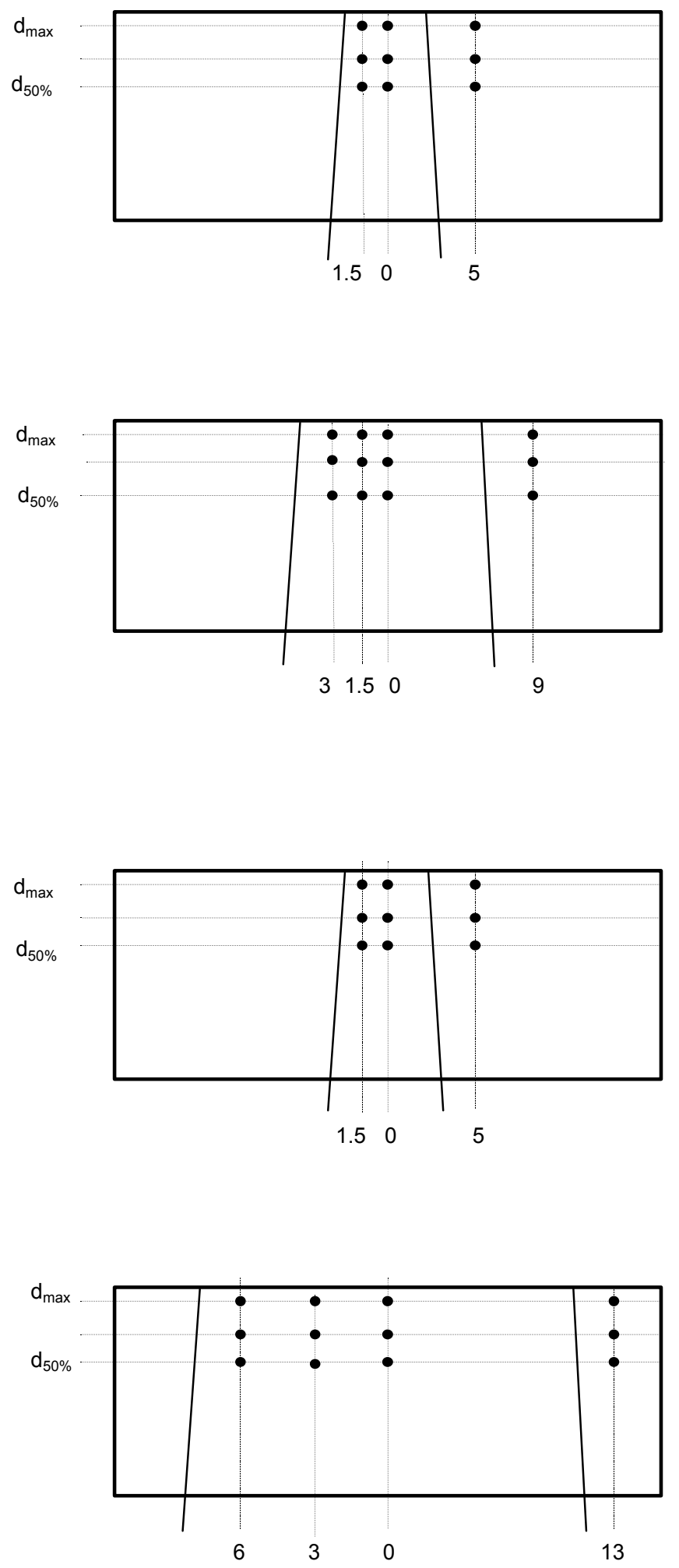

\section{TEST 1.a}

Field size $5 \mathrm{~cm} \times 5 \mathrm{~cm}$

$S S D=100 \mathrm{~cm}$

\section{TEST 1.b}

Field size $10 \mathrm{~cm} \times 10 \mathrm{~cm}$ $S S D=100 \mathrm{~cm}$

\section{TEST 2.a}

Field size $5 \mathrm{~cm} \times 20 \mathrm{~cm}$ $S S D=100 \mathrm{~cm}$

\section{TEST 2.b}

Field size $20 \mathrm{~cm} \times 5 \mathrm{~cm}$ $S S D=100 \mathrm{~cm}$

Fig. A.4.2.3 

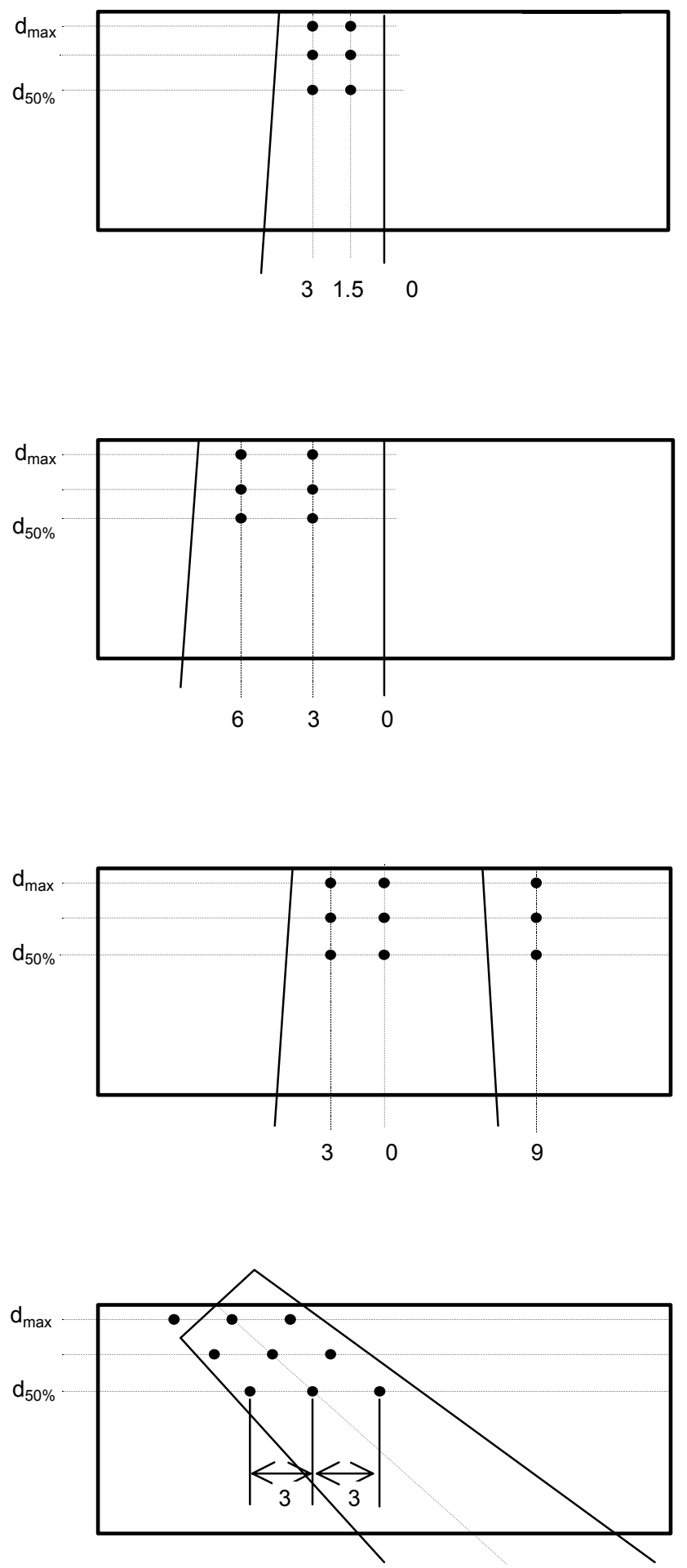

\section{TEST 3.a}

Field size $5 \mathrm{~cm} \times 10 \mathrm{cr}$ blocked to $5 \mathrm{~cm} \times 5 \mathrm{~cm}$ (half beam) SSD $=100 \mathrm{~cm}$

\section{TEST 3.b}

Field size $10 \mathrm{~cm} \times 20 \mathrm{c}$ blocked to $10 \mathrm{~cm} \times 10$ Half beam $S S D=100 \mathrm{~cm}$

\section{TEST 4}

Field size $10 \mathrm{~cm} \times 10 \mathrm{c}$ defined at $\mathrm{SSD}=110 \mathrm{~cm}$

\section{TEST 5}

Field size $10 \mathrm{~cm} \times 10 \mathrm{c}$ $\mathrm{SSD}=100 \mathrm{~cm}$ oblique incidence (gantry $45^{\circ}$ )

Fig. A.4.2.3 (continued) 

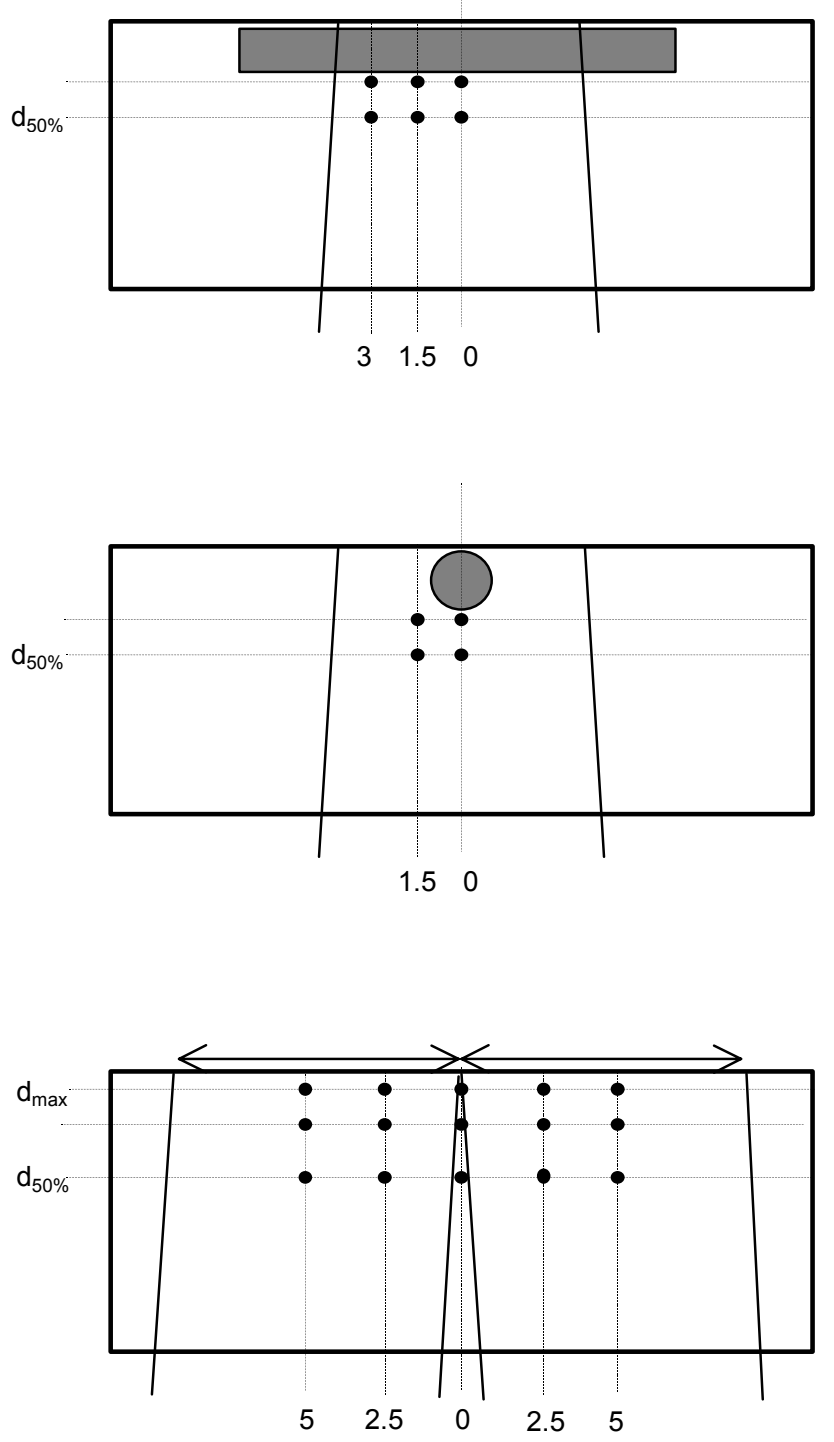

\section{TEST 6}

(For energies $>10 \mathrm{MeV}$ )

Field size $10 \mathrm{~cm} \times 10 \mathrm{~cm}$ $\mathrm{SSD}=100 \mathrm{~cm}$

$2 \mathrm{~cm}$ slab inhomogeneity: test 6a: air

test $6 \mathrm{~b}$ : bone density

\section{TEST 7}

(For energies $>10 \mathrm{MeV}$ )

Field size $10 \mathrm{~cm} \times 10 \mathrm{~cm}$ $\mathrm{SSD}=100 \mathrm{~cm}$

$2 \mathrm{~cm}$ cylinder inhomogeneity: test 7a: air

test $7 \mathrm{~b}$ : bone density

\section{TEST 8}

Field sizes $10 \mathrm{~cm} \times 10 \mathrm{~cm}$ abutting beams $\mathrm{SSD}=100 \mathrm{~cm}$ test 8a: electron + electron beam test $8 \mathrm{~b}$ : electron + photon beam

Fig. A.4.2.3 (continued) 


\section{A.5 Computation of sphere volume}

It is impossible to enter a sphere in a TPS, since spheres are defined by an infinite number of points in Cartesian grids. We can however approximate the sphere by defining the sphere in $2 N+1$ equidistant slices (with slice $-N$ and $N$ the tangent planes) and by $P$ points in each slice. The analytical volume within the above described tri-angulated surface is:

$$
V(R, N, P)=\left[\sum_{i=-N}^{N-1} \pi \int_{i^{*} R / N}^{(i+1)^{*} R / N}\left[f_{i}(x)\right]^{2} d x\right] \times\left[\frac{P}{2 \pi} * \sin \left(\frac{2 \pi}{P}\right)\right],
$$

with

$$
\mathrm{f}_{\mathrm{i}}(x)=\left[(i+1) \sin \frac{\mathrm{i}^{*} R}{N}-i \sin \frac{(\mathrm{i}+1) * R}{N}\right]+2\left(\sin \frac{(i+1) * R}{N}-\sin \frac{i * R}{N}\right) x,
$$

where $P$ is number of contour points in each slice and $(2 N+1)$ the number of slices in which the sphere is defined and $R$ the radius of the sphere. For $P$ and $N \rightarrow \infty$ this expression equals $4 / 3 \pi R^{3}$. 


\section{References}

[1] Westermann CF, Mijnheer BJ, van Kleffens HJ. Determination of the accuracy of different computer planning systems for treatment with external photon beams. Radiother Oncol 1: 339-347, 1984.

[2] Rosenow U, Dannhausen H-W, Luebbert K, Nüsslin F, Richter J, Robrandt B, Seelentag W-W and Wendhausen H. Quality assurance in treatment planning. Report from the German Task Group. In: The Use of Computers In Radiation Therapy, North-Holland, Elsevier Science Publishers BV, pp. 45-58, 1987.

[3] Wittkämper FW, Mijnheer BJ, van Kleffens $H$. Dose intercomparison at the radiotherapy centres in The Netherlands. Accuracy of locally applied computer planning systems for external photon beams. Radiother Oncol 11: 405-414, 1987.

[4] AAPM, American Association of Physicists in Medicine. Report 55: Radiation treatment planning dosimetry verification, Radiation Therapy Committee Task Group 23. American Institute of Physics, College Park, MD, USA, 1995.

[5] McCullough E, Holmes TW. Acceptance testing of computerized radiation therapy treatment planning systems: Direct utilization of CT scan data. Med Phys 12: 237242, 1985.

[6] Brahme A, Chavaudra J, Landberg T, McCullough E., Nüsslin F., Rawlinson A, Svensson $G$ and Svensson $H$. Accuracy requirements and quality assurance of external beam therapy with photons and electrons. Acta Oncol Suppl 1, 1988.

[7] Van Dyk J, Barnett RB, Cygler, JE and Shragge, PC. Commissioning and quality assurance of treatment planning computers. Int J Radiat Oncol Biol Phys 26: 261273, 1993.

[8] IPEMB, Institute of Physics and Engineering in Medicine and Biology. Report 68: A guide to commissioning and quality control of treatment planning systems. York, United Kingdom, 1996. 
[9] IPEM, Institute of Physics and Engineering in Medicine and Biology. Report 81: Physics aspects of quality control in radiotherapy. York, United Kingdom, 1999.

[10] SSRMP, Swiss Society for Radiobiology and Medical Physics. Report 7: Quality control of treatment planning systems for teletherapy. ISBN 3-908125-23-5, 1997.

[11] Fraass B, Doppke K, Hunt M, McCullough E, Nüsslin F, Rawlinson A, Svensson G and Svensson H. American Association of Physicists in Medicine Radiation Therapy Committee Task Group 53: Quality assurance for clinical radiotherapy treatment planning. Med Phys 25: 1773-1836, 1998.

[12] IAEA, International Atomic Energy Agency. Technical Report Series No. 430: Commissioning and quality assurance of computerized planning systems for radiation treatment of cancer. Vienna, Austria, 2004.

[13] IAEA, International Atomic Energy Agency. Report of a Team of Experts 26 May - 1 June 2001. Investigation of an accidental exposure of radiotherapy patients in Panama. Vienna, Austria, 2001.

[14] Mijnheer B, Olszewska A, Fiorini C, Hartmann G, Knoös T, Rosenwald JC, Welleweerd H. Quality assurance of treatment planning systems - practical examples for non-IMRT photon beams. ESTRO (European Society for Therapeutic Radiology and Oncology), Booklet no. 7 (Physics for Radiotherapy), ESTRO, Brussels, 2004.

[15] Mijnheer BJ, Battermann JJ and Wambersie A. What degree of accuracy is required and can be achieved in photon and neutron therapy? Radiother Oncol: 8:237-252, 1987.

[16] ICRU, International Commission on Radiological Units and Measurements. Report 24: Determination of absorbed dose in a patient irradiated by beams of $X$ and gamma rays in radiotherapy procedures. Washington, DC, USA, 1976.

[17] Hurkmans CW, Remeijer P, Lebesque JV and Mijnheer BJ. Set-up verification using portal imaging; review of current clinical practice. Radiother Oncol 58: 105-120, 2001. 
[18] Venselaar JLM, Welleweerd $\mathrm{J}$ and Mijnheer BJ. Tolerances for the accuracy of photon beam dose calculations of treatment planning systems. Radiother Oncol 60: 191-201, 2001.

[19] AAPM, American Association of Physicists in Medicine. Report 28: Quality assurance methods and phantoms for magnetic resonance imaging. American Institute of Physics, College Park, MD, USA, 1990.

[20] Hill DLG, Batchelor PG, Holden M and Hawkes DJ. Medical image registration. Phys Med Biol 46: R1-R45, 2001.

[21] Fraass BA and McShan DL. Three-dimensional photon beam treatment planning. In: Radiation Therapy Physics, Ed. A.R. Smith, Springer Verlag, pp.43-93,1995.

[22] IEC, International Electro technical Commission. Report nr. 1217: International standard. Radiotherapy equipment - coordinates, movements. Geneva, IEC publications, 1996.

[23] Siddon RL. Solution to treatment planning problems using co-ordinate transformations. Med Phys 8: 766-774,1981.

[24] McGee K P and Das IJ. Evaluation of digitally reconstructed radiographs (DRRs) used for clinical radiotherapy: a phantom study. Med Phys 22: 1815-1827, 1995.

[25] Craig T, Brochu D, Van Dyk J. A quality assurance phantom for three-dimensional radiation treatment planning. Int J Radiat Oncol Biol Phys 44: 955-966, 1999.

[26] Venselaar JLM and Welleweerd J. Application of a test package in an intercomparison of the performance of treatment planning systems used in a clinical setting. Radiother Oncol 60: 203-213, 2001.

[27] van 't Veld AA. Analysis of accuracy in dose and position in calculations of a treatment planning system for blocked photon fields. Radiother Oncol 45: 245-251, 1997 
[28] Harms WB, Low DA, Wong JW and Purdy J A. A software tool for the quantitative evaluation of 3D dose calculation algorithms. Med Phys 25: 1830-1836, 1998.

[29] Low DA, Harms WB, Mutic S, Purdy JA. A technique for the quantitative evaluation of dose distributions. Med Phys 25: 656-661, 1998.

[30] ICRU, International Commission on Radiological Units and Measurements. Report 50: Prescribing, Recording and Reporting Photon Beam Therapy. Bethesda, MD, USA, 1993.

[31] ICRU Report 62: Prescribing, Recording and Reporting Photon Beam Therapy. Supplement to ICRU Report No. 50. International Commission on Radiation Units and Measurements, Bethesda, MD, USA, 1999.

[32] BIR, British Institute of Radiology. Central axis depth dose data for use in radiotherapy. Br J Radiol Suppl 11. London, UK, 1972.

[33] BIR, British Institute of Radiology. Central axis depth dose data for use in radiotherapy. Br J Radiol Suppl 17. London, UK, 1983.

[34] BIR, British Institute of Radiology. Central axis depth dose data for use in radiotherapy. Br J Radiol Suppl 25. London, UK, 1996.

[35] Khan FM. The Physics of Radiation Therapy 2nd edn, Chapter 10. Williams and Wilkins, Baltimore, MD, USA, 1994.

[36] Dutreix A, Bjärngard BE, Bridier A, Mijnheer, B, Shaw, JE and Svensson, H. Monitor unit calculation for high energy photon beams. ESTRO Booklet No. 3. ESTRO, Brussels, Belgium, 1997.

[37] Mijnheer BJ, Bridier A, Garibaldi C, Torzsok K and Venselaar JLM. Monitor unit calculation for high energy photon beams - Practical examples. ESTRO Booklet No. 6. ESTRO, Brussels, Belgium, 2001. 
[38] NCS, Netherlands Commission on Radiation Dosimetry. Report 12: Recommendations for the determination and use of scatter correction factors of megavoltage photon beams. Delft, The Netherlands,1998.

[39] Venselaar JLM, van Gasteren JJM, Heukelom S, Jager HN, Mijnheer BJ, van der Laarse $\mathrm{R}$, van Kleffens $\mathrm{HJ}$ and Westermann C. A consistent formalism for the application of phantom and collimator scatter factors. Phys Med Biol 44: 365$381,1999$.

[40] Storchi P and van Gasteren JJM. A table of phantom scatter factors of photon beams as a function of quality index and field size. Phys Med Biol 41: 563-571, 1996.

[41] Mills MD, Hogstrom KR, and Fields, R.S. Determination of electron beam output factors for a $20 \mathrm{MeV}$ linear accelerator. Med. Phys. 12: 473-476, 1985

[42] van 't Veld AA and Bruinvis IA, Influence of shape on the accuracy of grid-based volume computations, Med. Phys. 22: 1377-85, 1995

[43] Lyman JT. Complication probabilities as assessed from dose-volume histograms. Radiat Res 104: 13-19. Suppl., 1985.

[44] Kutcher GJ, Burman CM, Brewster L et al. Histogram reduction method for calculating complication probabilities for three dimensional treatment planning evaluations. Int $\mathrm{J}$ Radiat Oncol Biol Phys 21: 137-146, 1991.

[45] Huizenga $\mathrm{H}$ en Storchi PRM. The use of computed tomography number in dose calculations for radiation therapy, Acta Radiol. Onc. , 24: 509-519, 1985.

[46] Thomas SJ. Relative electron density calibration of CT scanners for radiotherapy treatment planning. Brit.J Radiol 72: 781-786, 1999.

[47] ICRU, International Commission on Radiological Units and Measurements. Report 42: Use of computers in external beam radiotherapy procedures with high-energy photons and electrons. Bethesda, MD, USA, 1987.

United Kingdom, 1996. 
[48] NCS, Netherlands Commission on Radiation Dosimetry. Report 9: Quality control of medical linear accelerators; current practice and minimum requirements. Delft, The Netherlands, 1996. 